\title{
SEMIGROUPS ON A HALF-SPACE
}

BY

\section{J. G. HORNE(1)}

Introduction. The study of topological semigroups on noncompact manifolds is certainly in its infancy. Such studies as do exist have, in one way or another, rather restrictive hypotheses. This work is no exception. Attention is devoted almost exclusively to topological semigroups whose underlying topological spaces are homeomorphic to the set of number triples $\{(x, y, z): x \geqq 0\}$, which we call a halfspace. Nevertheless, due partly to the richness of the structure of Lie groups in $E_{3}$ and partly, but to a lesser extent, to the richness of the topological structure of a half-space, the structure of semigroups on a half-space seems fairly rich and possibly justifies the hope that some of the significant ideas for the general study have emerged.

The study of semigroups on a half-plane was carried out in [8], with some additional results being obtained in [3]. The present work depends in a material way on both of these papers. It depends even more on [5], a paper which owes a spiritual debt to [8] again. For there, it was observed that if one can find points $x$ and $y$ in the boundary such that the left orbit through $x$ and the right orbit through $y$ of the maximal group is closed, then one has an idempotent, an observation which leads to the work in [5]. Actually, this dependence on [5] may be so great and the results in [5] so peculiar to the plane that immediate future progress in the area will be made by ignoring difficult transformation group questions and proceeding "formally".

The organization of the paper is as follows: In the section on preliminaries, we have included statements of results from [5] for convenience of reference, but also because we use forms of these results which, though proved were not stated explicitly. In addition, we have included some elementary, but useful results about the groups $\{g \in G: g x \in x G\}$ and $\{g \in G: x g \in G x\}$ because they did not seem to fit anywhere else and their proofs do not use later material (here and hereafter, " $G$ " is the generic name for the maximal group containing the identity; $L$ denotes the boundary of $G$ and $S$ the entire semigroup).

In $\S 2$ we say everything we can think of about a single idempotent $e$ in $L$. This section contains the unique result of any consequence which can be proved for an arbitrary dimensional half-space (and appears to be true for an arbitrary manifold

Presented in part to the Society, September 23, 1963 and November 6, 1967 under the titles Idempotents in semigroups in a half-space and Can multiplication on $\mathrm{Sl}$ (2) be extended; received by the editors June 6, 1969.

( ${ }^{1}$ This research was supported in part by the National Science Foundation.

Copyright 두 1970, American Mathematical Society 
with boundary). Namely, $e$ always belongs to the closure of the left isotropy group $G_{l}(e)$. The next result, that $G_{l}(e)$ is connected, is proved only for a half-space but it is pretty clear that it can be generalized a great deal, even if it is not unrestrictedly true. Using the results in the appendix, it is proved that if $G_{l}(e)$ is planar then $G_{l}(e)^{-}$is a half-plane. Thereafter, different combinations of $\operatorname{dim} G e$ and $\operatorname{dim} e G$ are considered. Some combinations determine $G$ completely and the combination $\operatorname{dim} G e=1, \operatorname{dim} e G=1$ and $G e \neq e G$ determines $S$ itself (see Theorem 2.5 and the remarks preceding and following it). One of the very useful results of this section is that the left orbit $G x$ of a point $x$ is closed if and only if $x G$ is closed.

In $\S 3$, the question is considered whether there is a semigroup on a half-space such that $G$ is isomorphic to the universal covering group $\mathrm{Sl}(2)$ of the $2 \times 2$ real matrices of determinant 1 . We do not settle whether there is such an example but show that if there is it has a two-sided zero 0 and $L^{2}=\{0\}$. Moreover, we determine both the left and right actions of $G$ on $L$ for any such semigroup.

$\S 4$ treats sets of idempotents. The set $\xi_{i j}$ is the set of all idempotents $e$ such that $\operatorname{dim} G e=i$ and $\operatorname{dim} e G=j$ except that $\xi_{11}$ includes only those $e$ such that in addition $G e=e G$ (this is because everything is determined by Theorem 2.5 if $G e \neq e G$ ). The number of conjugacy classes in $\xi_{12} \cup \xi_{21}$ does not exceed 2 and if $G$ is not commutative and there is no zero, the number of conjugacy classes in $\xi_{12} \cup \xi_{21}$ $\cup \xi_{22}$ does not exceed 2. The total number of conjugacy classes of idempotents is countable (except for semigroups satisfying the conclusions of Theorem 2.5), and if there is a two-sided zero and no nilpotent elements it is finite. These results are used to show that if there is a two-sided 0 then there is a one-parameter group $P$ which runs to 0 .

It is developed in $\S 5$ that all orbits, both left and right, are nicely embedded. For example, if $\operatorname{dim} G x=2$ then $(G x)^{-}$is the entire boundary $L$ or a half-plane; a one-dimensional orbit is closed, or its closure is a half-ray.

The radical $\mathscr{R}$ is defined in $\S 7$ to be the closure of the set of weakly nilpotent elements. Initially, $x$ is said to be weakly left nilpotent if $x^{n} \notin G x$ for some positive integer $n$ and weakly right nilpotent if $x^{n} \notin x G$. The two notions turn out to be equivalent and $n=2$ works if any integer does. The possibilities for the radical are determined if there is no two-sided zero. We have not seriously tried to determine the possibilities for $\mathscr{R}$ if there is a two-sided zero. However, if there is a zero 0 and $\mathscr{R}$ is nonempty there is a nonzero nilpotent element. Furthermore, the set $N$ of nilpotent elements is a closed two-sided ideal containing all left and all right nilpotent ideals and $N^{3}=\{0\}$.

In the final section, we determine all possible $G$ and $L$ for which the radical is empty. Actually, it is our belief that if the radical is empty, $G$ and $L$ determine $G \cup L$ so we believe we have determined all semigroups on a half-space with empty radical (compare this with work in [3]). However, we have not considered this matter very long and the statement remains no more than a belief. In any case, examples of all of the possibilities listed are actually provided. Two facts surprise 
us here: (1) if $S$ has a zero and no nilpotent elements then $S$ is commutative; (2) though the number of idempotents must be finite if $S$ has a zero and no nilpotent elements, it is not bounded. Furthermore, examples can all be obtained as subsemigroups of the cartesian product of sufficiently many copies of the multiplicative semigroup of nonnegative real numbers.

Almost all results after Theorem 2.3 depend on the main result of the appendix which asserts (in part): if a localiy compact semigroup $S$ is the union of a dense planar group $G$ and a line $L$ then $S$ is topologically a half-plane.

We have not attempted to identify important open questions. It bears repeating that no example has been given in which $G$ is isomorphic to $\mathrm{Sl}$ (2). It should also be mentioned that while the situation is probably nearly settled as far as the radicalfree case is concerned, the case with nonempty radical is not. It may be open to question how interesting this case is. We know of many instances of semigroups with radical, and have no doubt that all sorts of bizarre examples exist. However, while we have tried, we have not succeeded in finding any general method for constructing them.

1. Preliminaries. A semigroup on a half-space is a topological semigroup whose underlying space is homeomorphic to the set of number triples $\{(x, y, z): x \geqq 0\}$ and such that the subset corresponding to the set with $x>0$ forms a group. Our attention is directed almost exclusively to such semigroups (and their subsemigroups). Thus, unless the contrary is explicitly mentioned, the context of each theorem is that of a semigroup on a half-space. Standing notation for this, again unless the contrary is mentioned, is as follows: $S$ denotes the entire semigroup which is thus a semigroup with identity 1 . The maximal subgroup of $S$ containing 1 (and occupying the interior of $S$ ) is denoted $G$. Of course, $G$ is a Lie group whose underlying space is homeomorphic to $E_{3}$. The boundary of $G$ is denoted $L$ and is topologically a plane. If $A$ is a set, $A^{-}$denotes its closure, and it is our custom to refer to $A^{-} \backslash A$ as the boundary of $A$ and denote it $\partial A$.

One theorem (Theorem 2.1) is as easy to prove in higher dimensions as it is in dimension three and for it we need the notion of a semigroup on a half- $n$-space. The definition is directly analogous to the above: it is a topological semigroup whose underlying space is homeomorphic to $\left\{\left(x_{1}, \ldots, x_{n}\right): x_{1} \geqq 0\right\}$ and such that the set corresponding to $\left\{x: x_{1}>0\right\}$ forms a subgroup.

If $G$ is a subgroup of a semigroup $S$ and $x \in S$ then $G_{l}(x)=\{g \in G: g x=x\}$. The subgroup $G_{l}(x)$ is called the left isotropy subgroup of $G$ at $x$. The right isotropy group is defined similarly and is denoted $G_{r}(x)$.

It was proved in [5] that $L$ contains an idempotent. Since we repeatedly use not only this result but other results from [5] on which its proof depends, and sometimes forms of these results not explicitly stated there, it seems appropriate to review the proof of that result and include statements of these other results as they will be used here. 
Note first the purely algebraic fact that if $G$ is a group in a semigroup $S$, if $x \in S$ and $x^{2} \in G x \cap x G$ then $G x \cap x G$ is a group and in particular, $G x$ and $x G$ contain an idempotent. For if $x^{2}=g x=x h$ and $u=g^{-1} x$ then $u^{2}=\left(g^{-1} x\right)\left(g^{-1} x\right)$ $=\left(g^{-1} x\right)\left(x h^{-1}\right)=g^{-1}\left(x^{2}\right) h^{-1}=g^{-1}(g x) h^{-1}=x h^{-1}=g^{-1} x=u$ so $u$ is an idempotent in $G x \cap x G$. The proof that $G x \cap x G$ is a group is routine.

Second, if $G$ is dense in a semigroup $S$ then $x^{2} \in(G x)^{-} \cap(x G)^{-}$since, for example, $x \in G^{-}$so $x^{2} \in G^{-} x \subset(G x)^{-}$. These ideas appear in [8] and form the basis for interest in closed orbit theorems and hence the work in [5]. There it was stated and proved that any connected Lie group acting on a subset of the 2-sphere has a closed orbit. This result is sufficient to yield an idempotent in $L$ when $L$ is a plane. However, several somewhat sharper results were actually proved though not stated and we need these results in the sequel.

THEOREM 1.1 [5]. Suppose a connected Lie group $G$ acts as a transformation group on a topological space $X$. Suppose for some $x \in X, \operatorname{dim} G x=1$. Then there exists a one-parameter subgroup $P$ of $G$ such that $G x=P x$. Furthermore, a necessary and sufficient condition that a particular one-parameter subgroup $P$ of $G$ have the property that $G x=P x$ is that $P$ have no conjugates in the isotropy subgroup $G_{x}$ at $x$.

COROLlary 1.1.1. If a connected Lie group $G$ acts in the plane and Gx is a onedimensional orbit then $G x$ is homeomorphic to a line or a circle.

Proof. According to [2], if $P$ is a one-parameter group and $\operatorname{dim} P x=1$ then $P x$ is a line or a simple closed curve.

THEOREM 1.2 [5]. Suppose $G$ is a connected Lie group acting in the plane L. Suppose $x \in L$ and $\operatorname{dim} G x=1$. Then there exists an element $y \in(G x)^{-}$and a oneparameter subgroup $P$ of $G$ such that if $z \in(G y)^{-}$then $G z=P z$.

THEOREM 1.3 [5]. Let $P$ be a one-parameter group acting in the plane L. Let $x \in L$. Then there exists $y \in(P x)^{-}$such that Py is closed.

COROLlary 1.3.1. Suppose $S$ is a semigroup on a half-space with maximal group $G$ and boundary L. If $x \in L$ then $(G x)^{-} \cap(x G)^{-}$contains an idempotent. In fact, if $H$ is any sub-Lie group of $G$ and $x \in H^{-}$, then $(H x)^{-} \cap(x H)^{-}$contains an idempotent. In particular, if $H$ is not closed in $S, H$ contains an idempotent in its boundary.

We now list a few results which are not needed until somewhat later. However, their proofs are elementary, and they are used in several different parts of the paper.

Proposition 1.4. If $\operatorname{dim} G x=2$ then $x G \subset G x$; in particular, if $\operatorname{dim} G x=\operatorname{dim} x G$ $=2$ then $G x=x G$.

Proof. Relative to any subgroup $K$ of $G$, define the subsets

$$
K^{l}(x)=\{g \in K: g x \in x K\} \text { and } K^{r}(x)=\{g \in K: x g \in K x\} .
$$


Evidently $K^{l}(x)$ and $K^{r}(x)$ are subgroups of $K$ and $K x \cap x K=K^{l}(x) x=x K^{r}(x)$. For $K=G$ and $\operatorname{dim} G x=2, K^{r}(x)$ is an open subgroup of $G$ so coincides with $G$, whence $x G \subset G x$.

Proposition 1.5. If $x, y \in L$ are such that $\operatorname{dim} G y=2$ and $x G \cap G y \neq \varnothing$ then $x G \subset G y$. If $\operatorname{dim} x G=2$ also then $x G=G y$.

Proof. Since, by the preceding, $y G \subset G y$, the set $\{g \in G: x g \in G y\}$ is a subgroup of $G$ which is open since $\operatorname{dim} G y=2$ and is nonempty since $x G \cap G y \neq \varnothing$. Therefore, $x G \subset G y$. If $\operatorname{dim} x G=2$ also the hypotheses on $x$ and $y$ are symmetrical so $G y \subset x G$.

Proposition 1.6. If $x G \subset G x$ then $G_{r}(x)$ is normal. Thus if $\operatorname{dim} G x=2$ or if $x G=G x, G_{r}(x)$ is normal.

The easy proof is omitted.

Finally, it is convenient to have a list of all Lie groups whose underlying spaces are Euclidean three space $E_{3}$. Since these groups are simply connected, they are uniquely determined by their Lie algebras. An examination of the list of threedimensional Lie algebras given in [7] shows that the list is complete. For this list and elsewhere we let Af (1) denote the affine group of the line-that is, the group which can be represented as the set $\{(a, b): a>0\}$ with multiplication given by $(a, b)(x, y)=(a x, a y+b)$.

Lie groups on $E_{3}: 1$. The three-dimensional vector group $V_{3}$;

2. The nilpotent group of $3 \times 3$ matrices

$$
\left(\begin{array}{lll}
1 & x & y \\
0 & 1 & z \\
0 & 0 & 1
\end{array}\right)
$$

3. $R \times$ Af (1) where $R$ denotes the additive group of real numbeis;

4. The semidirect products $R \cdot V_{2}$ which have the representations

$$
\left(\begin{array}{ccc}
p_{11}(t) & p_{12}(t) & x \\
p_{21}(t) & p_{22}(t) & y \\
0 & 0 & 1
\end{array}\right)
$$

where the map

$$
t \rightarrow\left(\begin{array}{ll}
p_{11}(t) & p_{12}(t) \\
p_{21}(t) & p_{22}(t)
\end{array}\right)
$$

is an isomorphism from $R$ into the group of nonsingular $2 \times 2$ real matrices;

5. The simple group Sl (2) which is the simply connected covering group of the group sl (2) of $2 \times 2$ real matrices of determinant 1 .

2. The position of idempotents. As already mentioned, Theorem 2.1 is as easy to prove in dimension $n$ as in dimension 3 and is virtually unique in this paper in this respect, though Theorem 2.2 is surely capable of considerable generalization. 
Except for a few lemmas, it is the only theorem whose hypothesis is other than that $S$ is a semigroup on a half-space. Each of these theorems concerns the left isotropy group $G_{l}(e)$ of an idempotent. Obviously, one has analogous results for right isotropy groups $G_{r}(e)$. Generally, only one version of a theorem is stated. Its "left-right" dual is then regarded as an obvious consequence, frequently without explicit reference.

THEOREM 2.1. Suppose $S$ is a semigroup on an n-dimensional half-space. Let e be an idempotent in the boundary $L$ of $S$. Let $G_{l}(e)$ denote the left isotropy subgroup of $G$ at $e$. If $G e$ is locally Euclidean then $e \in G_{l}(e)^{-}$.

Proof. Suppose $G e$ has dimension $k$. Let $V$ be a neighborhood of $e$. Since $G e$ is locally Euclidean, there is a closed $k$-cell $K$ containing $e$ which forms a neighborhood of $e$ in $G e$. There is a neighborhood $U$ of $e$ such that $U \subset V$ and if $x \in U$ then $x e \in K$. Since $S$ is topologically the cartesian product of the nonnegative reals and a Euclidean space, there is a closed $(k+1)$-cell in $J S$ such that $J \cap L=K$. Choose a neighborhood $W$ of $e$ relative to $J$ with the following properties: (1) $W \subset U$; (2) The set $K \backslash W$ is a $k$-dimensional annulus surrounding $e$; (3) The union of $K \backslash W$ with the part of the relative boundary of $W \cap G$ which lies in $G$ is a $k$-cell $M$.

Let $\rho$ denote radial projection in $K$ from $e$ onto the relative boundary of $K$ (which is also the relative boundary of $M$ ). Let $\mu(x)=x e$ for $x \in M$. If there is no member $x$ of $M$ such that $x e=e$ then $\rho \mu$ is well defined and is a retraction from $M$ onto its boundary. Since this is impossible, there is an element $x \in M$ such that $x e=e$. If $x \in G e \cap M$ then $x e \neq e$ since then $x e=x$. Since $M \cap L \subset K \subset G e, x$ must belong to $G$. Thus, every neighborhood of $e$ contains points of $G_{l}(e)$ and the theorem is proved.

Corollary 2.1.1. Suppose $S$ is a semigroup on a three-dimensional half-space, and suppose $e$ is an idempotent in $L$. Then $e \in G_{l}(e)^{-}$.

Proof. In this case, $L$ is a plane. If $\operatorname{dim} G e=2$ then $G e$ is open. If $\operatorname{dim} G e=1$ then $G e$ is a simple closed curve or a line by Corollary 1.1.1. Thus, in any case $G e$ is locally Euclidean and the conclusion follows.

For the next theorem we need a lemma which is presumably well known. We include a proof since we do not know a reference.

Lemma. Let $M$ and $N$ be $n$-dimensional manifolds and let $f: M \rightarrow N$ be a one-to-one continuous function mapping onto. Then $f$ is a homeomorphism.

Proof. Let $x_{0} \in M$. Let $V$ be a Euclidean neighborhood of $f\left(x_{0}\right)$ in $N$. Let $W$ be a Euclidean neighborhood of $x_{0}$ such that $W^{-}$is compact and $f(W) \subset V$. Then $f(W)$ is a Euclidean subset of $V$ of the same dimension as $V$ so $f(W)$ is open in $V$ and hence in $N$. It follows that if $A$ is any open subset of $M$ containing $x_{0}$ then $f\left(x_{0}\right) \in f(A)^{0}$. Thus $f$ is an open map so $f$ is a homeomorphism. 
THEOREM 2.2. Let $S$ be a semigroup on a half-space with boundary L. For each idempotent $e \in L, G_{l}(e)$ is connected.

Proof. Since $L$ is a plane, $G e$ is a manifold (being either a point, one-dimensional and therefore homeomorphic to a line or simple closed curve or two-dimensional and therefore an open subset of $L$ ). Now a one-to-one continuous function from one manifold onto another preserves dimension since a space covered by a countable number of closed sets of dimension $n$ is of dimension $n$. Thus the map $g G_{l}(e) \rightarrow g e$ from $G / G_{l}(e)$ onto $G e$ is a one-to-one continuous function from one manifold onto another. By the lemma, it is a homeomorphism so $G e$ and $G / G_{l}(e)$ are homeomorphic.

We note next that $G \cup G e$ is connected and locally arcwise connected. It is connected since it is contained between $G$ and $G^{-}$. To show that it is locally arcwise connected, it is clearly sufficient to show that each point of $\mathrm{Ge}$ is contained in arbitrarily small, arcwise connected neighborhoods. Let $x \in G e$. There are arbitrarily small neighborhoods of $x$ which are homeomorphic to the cartesian product of an arc and a cell. Let $V$ be such a neighborhood. Thus, if $y$ is any point of $G e \cap V$, there is an arc $A$ contained in $V$ such that $A \cap L=\{y\}$. Therefore if $y \in G e \cap V$, there are arcs $A_{1}, A_{2}$ contained in $V$ such that $A_{1} \cap L=\{x\}$ and $A_{2} \cap L=\{y\}$. Since $V \cap G$ is clearly connected, the endpoints of $A_{1}$ and $A_{2}$ which are contained in $G$ can be joined by an arc to yield an arc in $V \cap(G \cup G e)$ from $x$ to $y$. Thus $G \cup G e$ is locally arcwise connected.

Finally, $G \cup G e$ is simply connected. For this, it is enough to see that any closed curve in $G \cup G e$ which begins and ends at a point in $G$ can be continuously deformed in $G \cup G e$ to this point. But any such arc can be continuously deformed to an arc contained entirely in $G$ and is hence homotopic to a constant map.

Since the map $g \rightarrow g e$ is a retraction of $G \cup G e$ onto $G e, G e$ is simply connected. Therefore $G / G_{l}(e)$ is simply connected. But by $[9$, p. 617], the fundamental group of $G / G_{l}(e)$ is isomorphic to the factor group of $G_{l}(e)$ and its component of the identity. Therefore $G_{l}(e)$ is connected.

Corollary 2.2.1. Suppose $e$ is an idempotent in $L$ such that $\operatorname{dim} G e=2$. Then $G e$ is a plane and $G_{l}(e)$ is a line such that $G_{l}(e)^{-}=G_{l}(e) \cup\{e\}$. In fact, $G_{l}(e)^{-}$is isomorphic to the multiplicative semigroup of nonnegative real numbers.

Proof. Since $\operatorname{dim} G e=2, \operatorname{dim} G_{l}(e)=1$. The component of the identity of $G_{l}(e)$ is a one-dimensional Lie group. By the theorem, $G_{l}(e)$ is connected. No group on $E_{3}$ contains a nontrivial compact subgroup so $G_{l}(e)$ is isomorphic to the group of positive real numbers. That $G_{l}(e)^{-}=G_{l}(e) \cup\{e\}$ follows from [4] since $e$ is a zero for $G_{l}(e)^{-}$. That a locally compact semigroup which is the union of a dense oneparameter group and a zero is isomorphic to $[0, \infty)$ is proved in the appendix.

COROllary 2.2.2. No orbit of $G$ is a simple closed curve. If $e$ is an idempotent in $L$ such that $\operatorname{dim} G e=1$ then $G e$ is a line and $G_{l}(e)$ is a plane. 
Proof. A simple closed curve orbit would be compact. Since the closure of a left orbit of $G$ is a left ideal, it would be a subsemigroup which would hence be generated by an idempotent. Since $G_{l}(e)$ is connected, $G e$ is simply connected. In general, if $\operatorname{dim} G e=1$, then, $\operatorname{dim} G_{l}(e)=2$ so $G_{l}(e)$ is a connected two-dimensional Lie group without nontrivial compact subgroups and so is planar.

THEOREM 2.3. Let $e$ be an idempotent in $L$ such that $\operatorname{dim} G e=1$. Let $H$ be the component of the identity of $G_{l}(e)$. Then $H^{-}$is a half-plane.

Proof. We have already shown that there exists a one-parameter subgroup $P$ of $G$ and a planar subgroup $H$ of $G$ such that $G e=P e, e \in H^{-}$and $H e=e$. Let $P$ act on $S$ by left multiplication. There is an $\operatorname{arc} B$ in $P$ which forms a closed symmetric neighborhood of 1 in $P$ and is such that if $s, t \in B$ and $s e=t e$ then $s=t$. Therefc ' $\mathrm{e}$, by [12], there is a closed neighborhood $W$ of $e$ in $S$ and a closed subset $D$ in $W$ such that the map $(b, d) \rightarrow b d$ is a homeomorphism from $B \times D$ onto $W$.

In effect, we show that we may take $e \in D$. More precisely, let $e=b d$ and let $E$ be the component of $(B D) \cap L$ which contains $e$. Let $D_{1}$ be the component of $D \cap L$ which contains $d$. Then $B D_{1}=E$. Since $E$ is closed it is locally compact. Therefore, $D_{1}$ is either an arc, a simple closed curve or a half-ray. In any case, $b$ is not an endpoint of $B$. Let $A=B d^{-1}$ and let $C=b D$. Thus $A$ is an arc which forms a neighborhood of 1 in $P, C$ is a closed subset of $W$, the map $(a, c) \rightarrow a c$ from $A \times C$ onto $W$ is a homeomorphism and $e \in C$.

The next step in the proof is to show that there is an arc $K$ forming a neighborhood of 1 in $P$ and a neighborhood $V$ of $e$ in $S$ such that $H^{-} \cap(K c)$ consists of a single point if $c \in V \cap C$. To this end, the following lemmas are useful. For these lemmas and hereafter, we assume, as we obviously may, that $A$ is not only an arc of $P$ having the above properties but also, that right multiplication by $e$ is one-toone on $A$ and that $A$ is symmetric about 1 .

Lemma. If $M$ is any neighborhood of 1 in $A$ then there exists a neighborhood $V$ of $e$ such that if $t \in A, c \in V \cap C$ and tce $=e$ then $t \in M$.

Proof. If the lemma is false, there is a neighborhood $N$ of 1 in $A$ such that for every neighborhood $V$ of $e$ in $S$, there exists $t \in A \backslash N$ and $c \in V \cap C$ such that $t c e=e$. Thus there exist sequences $\left\{t_{n}\right\}_{n}$ and $\left\{c_{n}\right\}_{n}, t_{n} \in A \backslash N, c_{n} \in C$, such that $c_{n} \rightarrow e$ and $t_{n} c_{n} e=e$. We may assume $t_{n} \rightarrow t$ for some $t \in A, t \neq 1$. But since $t_{n} c_{n} e \rightarrow t e$ and $t_{n} c_{n} e=e$, this implies $t e=e$ which is a contradiction since right multiplication by $e$ on $A$ is one-to-one and $t \neq 1$.

Lemma. There is a neighborhood $Z$ of $e$ in $S$ and a neighborhood $K$ of 1 in $P$ such that right multiplication by e is one-to-one on $K c$ if $c \in Z \cap C$.

Proof. Choose and keep fixed one of the linear orders on $P$ compatible with its topology. Since $A$ is symmetric, $A=\left[a^{-1}, a\right]$ for some $a \in P, 1<a$. Take $x \in P$ such that $1<x<x^{2}<a$. Then

$$
\bigcap\left\{A t: t \in\left(x^{-1}, x\right)\right\} \supset\left(x^{-1}, x\right) .
$$


By the previous lemma, there is a neighborhood $V_{1}$ of $e$ in $S$ such that if $c \in V_{1} \cap C$ and $t c e=e$ then $t \in\left(x^{-1}, x\right)$.

Now $P e$ is a line. Since for any $c, A c e \subset P e$ and since, if $c$ is sufficiently close to $e$, Ace must nearly coincide with $A e$, there is a neighborhood $V_{2}$ of $e$ in $S$ such that if $c \in V_{2} \cap C$ then $e \in A c e$.

Let $Z=V_{1} \cap V_{2}$ and suppose $c \in Z \cap C$. Then right multiplication by $e$ is oneto-one on $\left(x^{-1}, x\right) c$. For, on the one hand, $c \in V_{2} \cap C$ so $e=t c e$ for some $t \in A$. Therefore right multiplication by $e$ is one-to-one on Atc since if $a_{1} t c e=a_{2}$ tce then $a_{1} e=a_{2} e$ so $a_{1}=a_{2}$. On the other hand, since $c \in V_{1} \cap C$ and $t c e=e, t \in\left(x^{-1}, x\right)$ so $\left(x^{-1}, x\right) \subset A t$. Therefore $\left(x^{-1}, x\right) c \subset A t c$, and since right multiplication by $e$ is one-to-one on Atc, it is certainly so on $\left(x^{-1}, x\right) c$. Thus take $K=\left(x^{-1}, x\right)$.

It now follows that if $H^{-} \cap K c \neq \varnothing$ and $c \in Z \cap C$ then $H^{-} \cap K c$ consists of a single point. For if $y \in H^{-}$then $y e=e$ while there is at most one point $u \in K c$ such that $u e=e$.

Our next goal is to show that all sufficiently small neighborhoods of $e$ in $L$ have points of $\mathrm{H}^{-}$on both sides of $\mathrm{Ae}$. We first observe that $\mathrm{H}^{-} \backslash H$ cannot consist of $e$ alone. For if the closure of $H$ consists of a single additional point, it must be compact, which is impossible.

Thus, choose $x \in H^{-} \backslash H$ but $x \neq e$. Since $e$ is a right zero for $H^{-}, e=x e \in x H^{-}$ $\subset(x H)^{-}$. Thus, every right orbit $H x$ of $x$ in $H^{-} \backslash H$ has $e$ in its closure. In particular, $x H \neq x$ so the dimension of $x H$ is 1 or 2 . But if $\operatorname{dim} x H=2$ then $x H$, being homogeneous, is open in $L$. Since $e \in(x H)^{-}, x H \cap K c$ must contain more than a single point if $c \in C \cap L$ and $c$ is sufficiently close to $e$. This is contrary to the previous lemma, so $\operatorname{dim}(x H)=1$.

Since $\operatorname{dim} x H=1$, there is a one-parameter subgroup $Q$ of $H$ such that $x H=x Q$. If $e \in x Q$, then $x Q$ contains an open arc containing $e$. Since $H^{-}$, and hence $x Q$, can intersect $K c$ at most once if $c$ is an element of $C$ sufficiently close to $e$, it follows that $x Q$ (and hence $H^{-}$) has points on both sides of $A e$.

Suppose, then, that $e \notin x Q$. Hence $x Q$ is not a simple closed curve, so $x Q$ is a line. If there are no other points of $H^{-} \cap L$, then the boundary of $H$ in $S$ is a half-ray. By the result in the appendix, $H^{-}$is topologically a plane. We shall show that this is impossible.

Let $R$ be a closed half-ray in $H^{-}$which emanates from $e$ and is otherwise contained in $H$. Let $D$ denote one of the components of $H^{-} \mid(R \cup x Q)$. Thus $D^{-}$is a half-plane. Now all sufficiently small neighborhoods of $x$ in $S$ are separated by $D^{-}$ into two components. This is also true of all sufficiently small $L$-neighborhoods of $x$. Since $e \in(x Q)^{-}$, we may assume that $x$ belongs to $Z$ and that $x=t_{1} c_{1}$ for some $t_{1} \in K, c_{1} \in C$. Thus there is a neighborhood $V$ of $x$ in $S$ which is contained in $Z$ and is such that $D^{-}$separates $V$ into two components. Since there are arbitrarily small neighborhoods of $x$ in $S$ which are products of an arc in $K$ and a closed neighborhood of $c$, in $C$, at most a change in name is involved in assuming $V \supset K C$. Now, as we have seen, the component of $(K C) \cap L$ which contains $x$ contains a 
neighborhood $K C_{1}$ of $x$ in $L$ where $C_{1}$ is an arc in $C$ containing $c_{1}$. Since $x Q$ can cross the sets $K c$ at most once, the endpoints of $K c_{1}$ lie on opposite sides of $D^{-} \cap L$ in $V \cap L$ and hence of $D^{-}$in $V$. Therefore there are $S$-neighborhoods $W_{1}, W_{2}$ of these endpoints such that if $y \in W_{1}$ and $z \in W_{2}$ then $y$ and $z$ are on opposite sides of $D^{-}$. Let $\pi$ be the map such that $\pi(t c)=c$. Then $\pi\left(W_{1} \cap(K C)\right)$ and $\pi\left(W_{2} \cap(K C)\right)$ are neighborhoods of $c_{1}$ in $C$. Let $C_{2}$ be a neighborhood of $c_{1}$ in $C$ which is contained in the intersection of these two. Then $K C_{2}$ is a neighborhood of $x$ in $S$ such that if $c \in C$ and $K c$ intersects $K C_{2}$ then $K c$ has its endpoints on opposite sides of $D^{-}$and hence intersects $D^{-}$. An analogous argument shows that there is a neighborhood $V$ of $x$ in $S$ such that if $K c$ intersects $V$ then $K c$ intersects the complementary component of $D$ in $H^{-} \backslash(R \cup x Q)$. But then there exists $c$ in $K C_{1} \cap V \cap Z$ such that $K c$ intersects both of these components and hence intersects $H$ at least twice, which is a contradiction.

We have shown that the boundary of $H$ in $L$ does not consist of $x Q$ and $e$ alone. Let $y \in H^{-} \cap L$ but $y \notin x Q$ and $y \neq e$. We know that $e \in(y H)^{-}$. If $y H$ is on the same side (locally) of $A e_{2}$ as is $x H$ then we may suppose $y \in K C$. This means that for some $c \in C$, there exist elements $t_{1}$ and $t_{2}$ in $K$ such that $y=t_{1} c$ and $x=t_{2} c$. But $K c$ intersects $H^{-} \cap L$ at most once so this is impossible. Thus $x H$ and $y H$ lie on opposite sides of $A e$ (more precisely, $x H$ and $y H$ enter a small neighborhood of $e$ on opposite sides of $A e$ ).

The previous conclusion is based on the assumption that $e \notin x H$. In this case, it follows that $H^{-} \cap L=(x H) \cup(y H) \cup\{e\}$. For let $z \in H^{-}$. Then $e \in(z H)^{-}$. However, $z H$ can approach $e$ only through $x H$ or $y H$ so $z \in(x H) \cup(y H)$. Suppose, on the other hand, $e \in x H$. Then $H^{-} \cap L=x H$. For, again if $z \in H^{-} \cap L$ then $e \in(z H)^{-}$. But $z H$ can approach $e$ only through one of the two sides of $x H$ so $z H=x H$ and $z \in x H$. Since $x H=e H$ and since $G_{l}(e)$ is connected, $x H$ is not a simple closed curve. Therefore $x H$ is a line. Thus, in either case, $H^{-} \cap L$ is a line. Hence, by the results in the appendix, $H^{-}$is a half-plane.

COROLlaRY 2.3.1. Let $e \in L$ be as in the theorem. Let $P$ be a one-parameter subgroup of $G$ such that $G e=P e$ and let $T$ denote the boundary of the left isotropy group $H$ of $e$. Then PT is a neighborhood of $e$ in $L$.

Proof. According to the proof of the theorem, there is an arc $C$ containing $e$ and contained in $L$ and an $\operatorname{arc} A$ which forms a symmetric neighborhood of 1 in $P$ such that the map $(a, c) \rightarrow a c$ is a homeomorphism from $A \times C$ onto $A C$ and $A C$ is a neighborhood of $e$ in $L$. Thus there exists an $L$-neighborhood $V$ of $e$ such that if $x \in T \cap V$ then $x=a_{x} c_{x}$ for unique elements $a_{x} \in A$ and $c_{x} \in C$. The set

$$
\left\{a_{x} \in A: x \in T \cap V\right\}
$$

is bounded away from the endpoints of $A$ if $V$ is sufficiently small. Thus

$$
\bigcap\left\{A a_{x}: x \in T \cap V\right\}
$$

contains a neighborhood $B$ of 1 in $P$. Hence, if $C_{0}=\left\{c_{x} \in C: x=a_{x} c_{x}\right.$ for some 
$x \in T \cap V$ and $\left.a_{x} \in A\right\}$, then $A T \supset \bigcup\{A x: x \in T \cap V\}=\bigcup\left\{A a_{x} c_{x}: x \in T \cap V\right\}$ $\supset B C_{0}$. It remains to show that $C_{0}$ is a neighborhood of $e$ in $C$. But if an element $c \in C$ is sufficiently close to $e$ then the endpoints of $A c$ are near those of $A e$ so $A c \cap T \neq \varnothing$. Thus all elements of $C$ which are sufficiently close to $e$ belong to $C_{0}$ so $C_{0}$ is a neighborhood of $e$ in $C$. Thus $A T$ is a neighborhood of $e$ in $L$ and hence so is $P T$.

For each of the next two theorems, we need the following facts about a semigroup $H^{-}$on a half-plane (see [8] for details). If $H^{-} \backslash H$ contains an idempotent $e$ such that $H e=e$ and $e H \neq e$ then, of course, $H$ is not commutative. Furthermore, $e H$ coincides with the entire boundary $H^{-} \backslash H$ of $H^{-}$. In addition, every element of $H^{-} \backslash H$ is an idempotent and in fact a right zero for $H^{-}$.

THEOREM 2.4. Suppose $e \in L$ is an idempotent such that $\operatorname{dim} G e=\operatorname{dim} e G=1$. Let $H$ be the left isotropy group of $e$ and let $J$ be the right isotropy group of e. Then the following conditions are equivalent:

(1) $H=J$;

(2) $G e=e G$;

(3) $H$ is a normal subgroup of $G$;

(4) $J$ is a normal subgroup of $G$;

(5) $H$ is conjugate to $J$.

If any of these conditions holds then Ge is a subgroup of L isomorphic to the additive group of real numbers.

Proof. We make the preliminary remarks that, in any case, according to Theorems 2.2 and 2.3,e $\in H^{-}, e \in J^{-}$and $H^{-}$and $J^{-}$are semigroups on a half-plane. Thus, if $H \neq J$, neither $H$ nor $J$ is commutative and the previous remarks about semigroups on a half-plane apply (except that every element of $J^{-} \cap L$ is a left zero for $J^{-}$).

To begin the proof of the theorem, suppose $H=J$ so $H e=e=e H$. Let $P$ be a one-parameter subgroup of $G$ having no conjugate in $H$. Thus $G e=P e$ and $e P=e G$.

Let $F_{1}=\{p \in P: p e \in e P\}$ and let $F_{2}=\{p \in P: e p \in P e\}$. As we have seen $F_{1}$ and $F_{2}$ are subgroups of $P$ and $F_{1} e=P e \cap e P=e F_{2}$. Furthermore the map which assigns an element $p$ to $q$ if and only if $p e=e q$ is an isomorphism from $F_{1}$ onto $F_{2}$. Assume $P e \neq e P$. Then $F_{1}$ is a proper subgroup of $P$. For otherwise, $P e \subset e P$ so $P e \cap e P$ is a neighborhood of $e$ in $e P$. Thus $e F_{2}$ is a neighborhood of $e$ in $e P$, whence $F_{2}$ is a neighborhood of 1 in $P$. Thus $F_{2}=P$ so $e P \subset P e$ which is a contradiction.

If $F_{1}$ is a proper subgroup of $P$, it is either closed, discrete and cyclic, or dense. If $F_{1}$ is dense, it is not cyclic, hence $F_{2}$ is not cyclic, hence $F_{2}$ is dense. But then $F_{1} e$ and $e F_{2}$ are dense in $P e$ and $e P$ respectively. Thus $P e$ and $e P$ are a pair of distinct topological lines such that $P e \cap e P$ is dense in each. This is impossible, so $F_{1}$ and $F_{2}$ are cyclic subgroups of $P$.

It follows that $P e \cap e P$ is discrete in $P e$ and in $e P$. Therefore there is a neighborhood $V$ of $e$ such that $V \cap P e \cap e P=\{e\}$. In fact, we may suppose $V \cap(P e)^{-}$ 
$\cap(e P)^{-}=\{e\}$ since no point of $P e$ is a limit point of either end of $P e$ (and no point of $e P$ is a limit point of either end of $e P$ ). If $p \in P$ is sufficiently close to 1 then epe $\in V$. Furthermore, epe $\in(P e)^{-} \cap(e P)^{-}$since $(P e)^{-}$is a left and $(e P)^{-}$is a right ideal. (These last two statements are true since $G e=P e$ and $e G=e P$.) By the previous corollary, if $p$ is sufficiently near 1 then $e p=q x$ for some $q \in P$ and $x \in T$ (where $T=H^{-} \cap L$ ). Since $e$ is a two-sided zero for $H^{-}, x e=e$ so $e p e=q x e=q e \in P e$ $\subset(P e)^{-}$. On the other hand, epe $\in(e P)^{-}$. Thus, if $p \in P$ is sufficiently close to 1 , epe $\in V \cap(P e)^{-} \cap(e P)^{-}$and hence epe $=e$. Using the above notation, epe $=q e$ so $q=1$. Thus $e p=x$. However, $e(e p)=e p$ and $e x=e$ so $e p=e x$ and hence $e p=e$. This is a contradiction since if $p \in P$ is different from $1, e p \neq e$.

We have shown that $P e=e P$ which completes the proof that (1) implies (2).

If $G e=e G$ then $G e$ is a group and $G$ commutes elementwise with $e$. Thus $H e=e H=e$ so $H=J$. Also, if $g \in G$ then $g H^{-1} e=g H_{e g}^{-1}=g e g^{-1}=g g^{-1} e=e$ so $g H g^{-1}=H$ and $H$ is normal. Thus (2) implies (1), (3) and (4).

Suppose next that $H$ is normal but $H \neq J$. Then of course $H$ is not commutative since $e \in H^{-}$. Thus $H$ is embedded as a direct factor so we may choose $P$ above to be the center of $G$ and have that $G=P H=H P$. But then $G e=P e=e P=e G$ which implies that $G$ commutes elementwise with $e$ so $H=J$. Thus (3) implies (1) and (2). Similarly (4) implies (1) and (2).

Obviously, (1), and hence each of (2) through (4) implies (5).

Finally, suppose $H$ is conjugate to $J$. Let $g$ be an element of $G$ such that $H=g^{-1} J g$. Then the right isotropy group of $e g$ is $H$. But the left isotropy group of $e g$ is also $H$. Now eg is also an idempotent element. To see this, recall that $H$ is not commutative (unless we already know that $H=J$, in which case there is nothing to prove). Thus $e H=H^{-} \backslash H=H^{-} \cap L$ so $e H$ is a closed line in $L$. Since $e G=e P, e G$ is also a line containing $e H$. Thus, $e H=e G$ so $e g \in H^{-} \backslash H$ and, as we have seen, every element of $H^{-} \backslash H$ is an idempotent.

We may now apply the previous results concerning $e$ to the element $e g$. In particular, eg is an idempotent such that the right isotropy group of $e g$ coincides with the left isotropy group of $e g$. Therefore $G(e g)=e g G$, so $G$ commutes elementwise with $e g$ and hence with $e$. Thus $G e=e G$ and we see that (5) implies (1).

If any one of the five conditions (1)-(5) holds then $G e=e G$. We have already seen that then $\mathrm{Ge}$ is a group and, being one-dimensional, is isomorphic to the additive group of real numbers.

Before considering the consequences of the existence of an idempotent $e$ such that $\operatorname{dim} G e=1=\operatorname{dim} e G$ but $G e \neq e G$, we need to recall some facts about the semidirect product groups $R \cdot V_{2}$. As mentioned above, every such group may be represented as a group of $3 \times 3$ matrices as follows:

$$
\left(\begin{array}{ccc}
p_{11}(t) & p_{12}(t) & x \\
p_{21}(t) & p_{22}(t) & y \\
0 & 0 & 1
\end{array}\right)
$$


where the map

$$
P(t) \rightarrow\left(\begin{array}{ll}
p_{11}(t) & p_{12}(t) \\
p_{21}(t) & p_{22}(t)
\end{array}\right)
$$

is an isomorphism from the additive group of real numbers into the group of $2 \times 2$ nonsingular real matrices. If $P(t)$ is reducible, we may assume $p_{21}(t)=0$. If $P(t)$ is irreducible then there is a positive real number $\rho$ (where we may suppose $\rho<1)$ and a real number $\theta$ such that

$$
P(t)=\rho^{t}\left(\begin{array}{rr}
\cos t \theta & \sin t \theta \\
-\sin t \theta & \cos t \theta
\end{array}\right)
$$

Consider the reducible case in more detail. If the proper values of $P(t)$ are equal we may suppose that $P(t)$ has one of the forms

or

$$
P(t)=\left(\begin{array}{cc}
e^{t} & t e^{t-1} \\
0 & e^{t}
\end{array}\right)
$$

$$
P(t)=\left(\begin{array}{cc}
e^{t} & 0 \\
0 & e^{t}
\end{array}\right)
$$

If the proper values of $P(t)$ are unequal, we may suppose $P(t)$ has the form

$$
P(t)=\left(\begin{array}{ll}
e^{t} & 0 \\
0 & e^{a t}
\end{array}\right)
$$

where $a \neq 0$. (The case $a=0$ yields a Lie group of course but it isomorphic to $R \times \operatorname{Af}(1)$ which is not one of the semidirect products $R \cdot V_{2}$ since, by definition these must have commutator subgroup isomorphic to $V_{2}$.)

The case $a<0$ is distinctive: In the above representation it is the only one which yields a subgroup of Gl (3) which is closed in the entire semigroup of $3 \times 3$ real matrices. Indeed if, for the moment, $G$ denotes any one of the above groups except one with $a<0$ then $G^{-}$is a semigroup on a half-space having the semigroup

$$
\left(\begin{array}{lll}
0 & 0 & x \\
0 & 0 & y \\
0 & 0 & 1
\end{array}\right)
$$

for its boundary. (It is perhaps well to notice here that each of these semigroups contains an idempotent $e$ such that $\operatorname{dim} G e=2$ and $\operatorname{dim} e G=0$. This situation is considered in detail in Theorem 2.13.)

A flow has been called hyperbolic if, roughly speaking, it is contracting in one direction and expanding in another. We shall therefore call a group whose matrices have the form

$$
\left(\begin{array}{lll}
e^{t} & 0 & x \\
0 & e^{a t} & y \\
0 & 0 & 1
\end{array}\right)
$$

with $a<0$, a hyperbolic semidirect product. 
The distinctive character of hyperbolic semidirect products appears in the next theorem as well as in Theorem 2.13. At the conclusion of the proof of the next theorem we offer a collection of examples of the sort described in the theorem. We believe this collection to be exhaustive but postpone further consideration of this matter until a later paper.

THEOREM 2.5. Suppose $e$ is an idempotent in $L$ such that $\operatorname{dim} G e=\operatorname{dim} e G=1$. Let $H$ be the left isotropy group of $e$ and let $J$ be the corresponding right isotropy group. Assume that $H \neq J$. Then the following are true:

(1) For each $x, y \in L, x G$ and $G y$ are closed lines in $L$ such that $x G \cap G y=\{x y\}$.

(2) The left isotropy group of $x \in L$ is conjugate to $H$ and is distinct from the right isotropy group of $x$ which is conjugate to $J$.

(3) $G$ is a semidirect product $R \cdot V_{2}$ of the additive group $R$ of real numbers and the two-dimensional vector group $V_{2}$. Furthermore, $G$ contains at least two distinct conjugacy classes of nonnormal noncommutative planar groups and is nonhyperbolic.

Proof. We shall use again the information that $e \in H^{-}, e \in J^{-}$and that $H^{-}$and $J^{-}$are each noncommutative semigroups on a half-plane. Furthermore, by the preceding theorem, neither $H$ nor $J$ is normal and $H$ and $J$ are not conjugates. Since $H$ and $J$ are not commutative, every element of $H^{-} \cap L$ is an idempotent and a right zero for $H^{-}$while every element of $J^{-} \cap L$ is an idempotent and a left zero for $J^{-}$. Finally, $e G=e H=H^{-} \cap L=H^{-} \backslash H$ and $G e=J e=J^{-} \cap L=J^{-} \backslash J$. Thus $e G$ is a right ideal and $G e$ is a left ideal in $S$.

It follows that $G e \cap e G=\{e\}$. For let $x \in G e \cap e G$. Then $x \in G e=J^{-} \cap L$ so $e x=e$. But $x \in e G$ so $e x=x$, hence $x=e$. As a consequence, $e G \cdot G e=e G e=e$ since $e G \cdot G e \subset e G \cap G e$.

Now let $x$ be an arbitrary element of $L$. Since $e G$ is a right ideal, ex $=e g$ for some $g \in G$. We intend to show that the right isotropy group of $x$ is $g^{-1} J g$, that $x \in\left(g^{-1} J g\right)^{-}$, that $G x=G e g$ and that $G x \cap e G=\{e g\}$. Analogous results hold for $x e$. The two sets of results together yield the first conclusion with the " $x$ " of statement (1) replaced by $e$, and $y$ of that statement replaced by the present $x$. It follows that $x$ then satisfies the same hypotheses as $e$. Thus the first conclusion as stated follows by treating $e$ and $x$ generically.

We prove the above assertions in a series of steps labeled "Notes".

Note 1. Assume first that ex $=e$. Then $x G \cap$ Ge is empty unless $x \in G e\left(=J^{-} \cap L\right)$.

For suppose $x g=h e$ for some $g, h \in G$. Then $\operatorname{exg}=e h e=e$ so $e g=e$. Therefore $g \in J$. Now $g \in J$ and $x g=h e$ imply $x=h e g^{-1}=h e \in G e$.

Note 2. If ex=e then $x G \cap G e$ is not empty so $x \in J^{-} \cap L$.

For this result, we need to know that $e G$ has points on both sides of $G e$. Evidently $H$ has points on both sides of $J$ and, indeed, must have points on both sides of $J$ which lie on nonnormal one-parameter subgroups of $H$. Each such subgroup has points in $H^{-} \cap L=e G$ in its boundary. Thus $e G$ has points on both sides of $G e$. 
Now $e$ belongs to the closure of a one-parameter subgroup $P$ of $H$ and $P^{-}=P$ $\cup\{e\}$. Let $[e, 1]$ denote the interval in $P^{-}$from $e$ to 1 . If $p \in[e, 1]$, let $f(p, z)=p z$ for $z \in L$. Thus $f(e,-)$ is left multiplication by $e$ and is thus a retraction of $L$ onto $e G$. Furthermore, $f(e, x G)=e G$ since $e x=e$. Since $e G$ has points on both sides of $G e$, there exists some $r \in(e, 1]$ such that $f(r, x G) \cap G e \neq \varnothing$. That is, there exist elements $g, h \in G$ such that $r x g=h e$. Hence $x g=r^{-1}$ he so $x G \cap G e \neq \varnothing$.

Note 3. Let $x \in L$. If ex=eg for some $g \in G$ then $x \in\left(g^{-1} J g\right)^{-}$and the right isotropy group at $x$ is $g^{-1} \mathrm{Jg}$.

We may assume $x \notin G e$ since if $x \in G e$, we may assume $g=1$ and the conclusion is already known. Since $e x=e g$, exg $g^{-1}=e$ so $x g^{-1} \in J^{-} \cap L$. Since every element of $J^{-} \cap L$ is a left zero for $J^{-}, x\left(g^{-1} J g\right)=\left(x g^{-1} J\right) g=x g^{-1} g=x$. Thus $g^{-1} J g$ is contained in the right isotropy group of $x$. Since $G_{r}(x)$ is connected, if $G_{r}(x) \neq g^{-1} J g$ then $G_{r}(x)=G$-that is $x G=x$. However, $x e \in(x G)^{-}$and $x e \neq x$ since $x e \in G e$ and $x \notin G e$ so this is a contradiction. Thus the right isotropy group at $x$ is $g^{-1} J g$.

To see that $x$ belongs to $\left(g^{-1} J g\right)^{-}$note that since $x g^{-1} \in J^{-}$,

$$
g^{-1}\left(x g^{-1}\right) g \in\left(g^{-1} J g\right)^{-} \text {. }
$$

That is, $g^{-1} x \in\left(g^{-1} J g\right)^{-} \cap L$. Since $J^{-} \cap L$ is a left ideal, $\left(g^{-1} J g\right)^{-} \cap L$ is a left ideal so $x \in\left(g^{-1} J g\right)^{-} \cap L$.

Note 4. If $x \in L$ then $G x \cap e G=\{e x\}$.

We know $e x=e g$ for some $g \in G$ since $e G$ is a right ideal. Now $e g$ is an idempotent, $H$ is the left isotropy group of $e g, g^{-1} J g$ is the right isotropy group of $e g$ and $H \neq g^{-1} J g$ by Theorem 2.4. The results so far quoted for $e$ are thus available for eg. In particular, $(G e g) \cap e G=\{e g\}=\{e x\}$. We want to see that $G x=G e g$. But $G e g=\left(g^{-1} J g\right)^{-} \cap L$ for the same reason that $G e=J^{-} \cap L$. Since $x \in\left(g^{-1} J g\right)^{-} \cap L$, $x \in G e g$ so $G x=G e g$.

Summarizing these results, we see that every $x \in L$ is idempotent and the right isotropy group $G_{r}(x)$ is a conjugate of $J$. Arguments dual to the above show that the left isotropy group of $x$ is conjugate to $H$ and hence distinct from $G_{r}(x)$. Thus everything proved above for $e$ is true for $x$. Translated, this means that if $x, y \in L$ then $x G$ and $G y$ are closed lines in $L$ and $G y \cap x G=\{x y\}$ which gives the first conclusion of the theorem. Along the way we have also gotten the second.

To prove the third conclusion, examine the list of the various Lie groups whose underlying space is $E_{3}$. From what has already been proved, $G$ must have at least two conjugacy classes of nonnormal noncommutative planar subgroups. This immediately rules out the two nilpotent groups and the simple group Sl (2). The only groups left are the various semidirect products $R \cdot V_{2}$ and $R \times \operatorname{Af}(1)$. But in the latter group, $R$ is central so every noncommutative planar group is normal. Thus $R \times \operatorname{Af}(1)$ is ruled out. Thus $G$ is one of the semidirect products $R \cdot V_{2}$ having at least two distinct conjugacy classes of noncommutative planar groups.

It remains to show that $G$ can not be a hyperbolic semidirect product. Let $P=H \cap J$ and let $Q_{1}$ denote the normal one-parameter subgroup of $H$ and let $Q_{2}$ 
denote the normal one-parameter subgroup of $J$. Of course $e \in P^{-}$. Furthermore, if $p$ is in the component of $P \backslash\{1\}$ which runs from $e$ to 1 then the map $x \rightarrow p x p^{-1}$ is contracting on $Q_{1}$ since $H^{-}$is isomorphic to $\operatorname{Af}(1)^{-}$. But the same statement holds for the map $x \rightarrow p x p^{-1}$ in $Q_{2}$. A hyperbolic semidirect product is characterized by the condition that if $p$ is restricted to one-component of $P \backslash\{1\}$ then its action $x \rightarrow p x p^{-1}$ on one normal one-parameter subgroup is contracting and in the other is expanding. Hence $G$ is not a hyperbolic semidirect product and the proof of the theorem is complete.

We next show that each nonhyperbolic semidirect product $R \cdot V_{2}$ which contains at least two conjugacy classes of nonnormal, noncommutative planar groups is the maximal group of a semigroup on a half-space which contains an idempotent $e$ satisfying the hypotheses of the previous theorem. If $G$ is such a group it evidently has a faithful representation as a group of $4 \times 4$ matrices of the following form:

$$
\left(\begin{array}{llll}
1 & a & 0 & 0 \\
0 & \lambda & 0 & 0 \\
0 & 0 & \lambda^{c} & b \\
0 & 0 & 0 & 1
\end{array}\right)
$$

where, since $G$ is nonhyperbolic, $c>0$ and where $\lambda$ is an arbitrary positive number and $a, b$ are arbitrary real numbers. Evidently the closure of this group, which consists of all such elements with $\lambda \geqq 0$ and $a, b$ arbitrary is a semigroup on a half-space. It is easy to check that if $x, y \in L$ then $\{x y\}=x G \cap G y$ so that the semigroup is of the sort described in the theorem.

THEOREM 2.6. Suppose L contains an idempotent $e$ such that $\operatorname{dim} G e=1$ and $\operatorname{dim} e G=2$. Then $G e \subset e G$ so Ge is a group and the left isotropy subgroup of $e$ is a noncommutative normal subgroup of $G$. Therefore $G$ is isomorphic to $R \times \operatorname{Af}(1)$ and $G e=P e$ where $P$ is the center of $G$.

Proof. By the results in the first section, we already know that $G e \subset e G$, that in general if $e$ is an idempotent then $G e \cap e G$ is a group and that when $G e \subset e G$, $G_{l}(e)$ is normal.

To obtain the conclusion about the nature of $G$, recall that $\operatorname{Af}(1)$ is complete (i.e. zero center and every automorphism an inner automorphism) and that a complete normal subgroup is embedded as a direct factor. The final conclusion is immediate since the center obviously has no conjugates in $H$.

REMARK. To see that the hypotheses of the theorem can obtain, consider the following example of a semigroup $S$ on the set $(x, y, z)$ with $x \geqq 0$ and multiplication defined by: $(a, b, c)(x, y, z)=(a x, b x+y, c+z)$. If $e=(0,0,0)$ then $\operatorname{dim} G e=1$ and $\operatorname{dim} e G=2$.

THeorem 2.7. Let $x \in L$. Then $G x$ is closed if and only if $x G$ is closed. In particular, if $e$ is an idempotent in $L$ such that $G e=e$ then $e G$ is a closed line if $\operatorname{dim} e G=1$ and $e G=L$ if $\operatorname{dim} e G=2$. 
Proof. If we prove that $G x$ being closed implies $x G$ is closed, the converse will follow since all results about left orbits of $G$ have analogues for the right orbits of $G$.

Suppose that $G x$ is closed. By Corollary 1.3.1, we may assume that $x$ is an idempotent $e$. We proceed, then, to consider the various combinations of $\operatorname{dim} G e$ and $\operatorname{dim} e G$.

Let $\operatorname{dim} G e=0$ so $G e=e$. If $\operatorname{dim} e G=0$, the conclusion is trivial. Assume $\operatorname{dim} e G \rightarrow 0$ but $e G$ is not closed. Then there is an element $y \in(e G)^{-} \mid e G$ such that $y G$ is closed. Since every left ideal must meet every right ideal, Ge $\cap y G$ is not empty so, since $G e=e, e \in y G$. Therefore $e G=y G$ so $y \in e G$ which is a contradiction. It follows that if $G e=e$ then $e G$ is closed. If $\operatorname{dim} e G=1$, then $e G$ is a line since it cannot be a simple closed curve. If $\operatorname{dim} e G=2$ then $e G=L$ since $e G$ is also open in this case.

Suppose next that $\operatorname{dim} G e=1$. If $\operatorname{dim} e G=0$ then again the conclusion is immediate. If $\operatorname{dim} e G=1$, there are two possibilities: If $G e=e G$ the conclusion is obvious. If $G e \neq e G$ then the conclusion follows from Theorem 2.5. If $\operatorname{dim} e G=2$ then it follows from the previous theorem that $G$ is isomorphic to $R \times \operatorname{Af}(1)$ and that $G e=P e$ where $P$ is the center of $G$. If $e G$ is not closed, then there exists an element $y \in(e G)^{-} \mid e G$ such that $y G$ is closed. As before, $G e \cap y G \neq \varnothing$. Since $G e=P e$, there exist elements $p \in P$ and $g \in G$ such that $p e=y g$. Therefore $e=p^{-1} y g$ $=y p^{-1} g$ since $p$ belongs to the center. Hence $e \in y G$ so $y \in e G$ which is a contradiction. Therefore, if $\operatorname{dim} G e=1, G e$ is closed and $\operatorname{dim} e G=2$ then $e G=L$ and $e G$ is closed.

The proofs of the desired results in the remaining cases are gotten by interchanging references to left and right orbits in the cases already considered.

TheOREM 2.8. Suppose $e$ is an idempotent in L such that $\operatorname{dim} G e=1$. Then either Ge is a closed line or Ge is a group, $G e=e G$ and $(\mathrm{Ge})^{-}$is a half-ray whose endpoint is a right zero for $S$. In more detail: if $\operatorname{dim} e G=0$ or if $\operatorname{dim} e G=1$ but $G e \neq e G$ then $G e$ is closed. If $\operatorname{dim} e G=2$ or if $G e=e G$ then Ge is a group and Ge is either closed or $(\mathrm{Ge})^{-}$is a half-ray whose endpoint is a right zero for $S$.

Proof. If $\operatorname{dim} e G=2$ or if $G e=e G$, we have already seen that $G e$ is a group isomorphic to the additive group of real numbers. By [2], if the boundary of $G e$ is not empty, then it is either a point or a simple curve and in the latter case, it consists of a single orbit of $\mathrm{Ge}$. It follows that $(\mathrm{Ge})^{-} \backslash \mathrm{Ge}$ consists of a single left orbit of $G$ so $(G e)^{-} \backslash G e$ contains but a single point-say $z$. Since $G z \subset(G e)^{-} \backslash G e$, $G z=z$ and $z$ is a right zero for $S$.

The conclusion in case $\operatorname{dim} e G=1$ but $G e \neq e G$ is stated in Theorem 2.5. If $\operatorname{dim} e G=0$ then $e G=e$ and $G e$ is closed by the previous theorem.

The next main result concerns the boundary of a two-dimensional orbit through an idempotent. Several times in its proof as well as later, we use a result which assures the existence of certain one-parameter groups which "run to the boundary", and accordingly state it as a preceding lemma. For it, we let $S$ be an arbitrary 
locally compact semigroup. Subsequently, $S$ again denotes a semigroup on a halfspace unless the contrary is specifically mentioned.

LEMMA 2.9. Let $S$ be a locally compact topological semigroup. Suppose $z$ is a right zero for $S$ such that every neighborhood of z contains an element which belongs to a group isomorphic to the additive group of real numbers. Then there exists a neighborhood $V$ of $z$ and a one-parameter group $P$ such that $\left(P^{-} \mid P\right) \cap V \neq \varnothing$. If $z$ is not the limit point of other idempotents then $P^{-}=P \cup\{z\}$, and in fact, every point $v \in V$ which is on a one-parameter group is on a one-parameter group $P$ such that $P^{-}=P \cup\{z\}$.

Proof. According to Lemma 2 of [2], if $V$ is a compact neighborhood of $z$ then there is a neighborhood $W$ of $z$ such that $V W \subset V$. Such a neighborhood has the property that if $x \in V \cap W$ then $x^{n} \in V$ for every positive integer $n$. If $x$ belongs to a group $P$ isomorphic to $R$ then $\left(P^{-} \mid P\right) \cap V \neq \varnothing$. For since $P$ is isomorphic to $R$, $\left\{x^{n}\right\}_{n}$ has no accumulation points in $P$. But since $V$ is compact, $\left\{x^{n}\right\}_{n}$ has an accumulation point in $V$.

For the second conclusion, note that the closure of $\left\{x^{n}\right\}_{n}$ is a compact semigroup, which thus contains an idempotent. Since we may assume that $V$ has no idempotents other than $z, z$ is an accumulation point for $\left\{x_{n}\right\}_{n}$. Therefore $z \in P^{-}$. Since $z$ is a right zero for $S, z$ is a two-sided zero for $P$. By [4], $P^{-}=P \cup\{z\}$.

Theorem 2.10. Let $e$ be an idempotent in $L$ such that $\operatorname{dim} G e=2$. Let $B$ denote the boundary of $G e$ and assume $B$ is not empty. Then $B$ is a two-sided ideal which is topologically a line or a half-ray. Furthermore, one of the following holds:

(1) for each $x \in B, G x=x$ and $x G=B$;

(2) for each $x \in B, x G=x$, and $G x=B$;

(3) if $x \in B$, then $G x=x G=B$ and $B$ is a group;

(4) $B$ contains a two-sided zero for $S$ and each component of $B \backslash\{0\}$ forms a left as well as a right orbit of $G$;

(5) $B$ is a half-ray whose endpoint is a two-sided zero for $S$ and $B^{2}=\{0\}$.

Proof. Since $G e$ is a plane, $B$ cannot consist of a single point. Also there are the following possibilities: either $G e=e G$ and $G e$ is a group, or $\operatorname{dim} e G=1, e G$ is a group and $e G \subset G e$. Clearly $B$ is a two-sided ideal in the first case. In the second case, $(G e) G=G(e G) \subset G(G e)=G e$. Thus $(G e)^{-} G \subset(G e)^{-}$so $B$ is a right ideal in this case also.

For reference later in the proof, we list the following additional facts about $\mathrm{Ge}$ : In any case, $G e$ is a semigroup, every element of $G e$ belongs to a one-parameter subgroup of $G e$ and no element of $B$ is a limit point of idempotents of $G e$. If $G e$ is a group, each of these results is immediate. Suppose $\operatorname{dim} e G=1$. Then $e G$ is a one-parameter subgroup of $G e, G$ is isomorphic to $R \times \operatorname{Af}(1)$ and $G_{l}(e)$ is one of the noncommutative planar groups of $G$. Let $H=G_{l}(e)$ and let $T=H^{-} \cap L$. Of course, $H^{-}$is a semigroup on a half-plane and since $H e=e$ but $e H \neq e, T=e H$. 
Thus, $T \subset G e$. Next, every element of $G e$ is on a conjugate of $e G$. For $g e \in g(e G) g^{-1}$. This shows that every element of $G e$ is on a one-parameter subgroup of $G e$ and that the only idempotents of $G e$ are in $T$. Since $T \subset G e$, no element of $B$ is a limit point of idempotents in $\mathrm{Ge}$.

We now proceed to determine the structure of $B$. Suppose first that $B$ contains an element $x$ such that $G x=x$ and $x G \neq x$. Then $x$ is a right zero for $S$ and $x G$ is a closed line. Invoking the previous lemma, we obtain a one-parameter subgroup $P$ of $G e$ such that $P^{-} \cap B \neq \varnothing$ (since every such $P$ in $G e$ is closed in $G e, P^{-} \mid P \subset B$ ). Furthermore, $P^{-} \mid P$ is a point or a simple closed curve. Suppose the latter holds. Then the identity $f \in P^{-} \mid P$ is such that $P^{-} \mid P=P f$. Therefore, $\operatorname{dim} G f=1$. But then $G f$ is a line which is impossible. Thus $P^{-} \mid P$ is a single point $w$ which is a zero for $P^{-}$and it follows from the appendix that $P^{-}$is isomorphic to $[0, \infty)$. We claim $w \in x G$. For, since $\{x\}$ is a left ideal, $x \in(w G)^{-}$so $\operatorname{dim} w G=1$. If $x \notin w G$ then $w G$ is not closed so $G w \neq w$ so $\operatorname{dim} G w=1$. Therefore $w G=G w$ and $G w$ is a group. Hence $(G w)^{-}$is a closed half-ray with $x \in(G w)^{-}$. But if $x \in(G w)^{-}$then

$$
x G \subset(G w)^{-} G \subset(G w G)^{-}=(G w)^{-}
$$

so the entire line $x G$ is in the closure of the half-ray $(G w)^{-}$which is absurd. It follows that $w \in x G$. Thus $P^{-}=P \cup\{w\}$ where $w G=x G$ and $G w=w$. Suppose there exists $y \in B \mid w G$. Then $y P^{-}=y P \cup\{y w\}=y P \cup\{w\}$. According to [1], $y P^{-}$is a half-ray from $w$ through $y$ in $B$. Since it is impossible for three closed half-rays from a point to lie in the boundary of a plane (in a plane), there is no such $y$ so $B=x G$ in this case.

If there exists an element $x \in B$ such that $x G=x$ but $G x \neq x$ then arguments similar to those just used show that $B=G x$.

Suppose next that neither of the previous cases holds and that $B$ contains an idempotent $f$ which is not a two-sided zero for $S$. Then $G f=f G$ and $G f$ is a onedimensional group. Hence, $G f$ is closed or $(G f)^{-}$is a half-ray which is the union of $G f$ and a two-sided zero. Assume first that $G f$ is closed. Since $G f=f G$ is a group, $G$ commutes elementwise with $f$. Let $H=G_{r}(f)$. Then $f H=f$ also so $f$ is a two-sided zero for the half-plane semigroup $H^{-}$. Thus there exists a one-parameter subgroup $P \subset H$ such that $P^{-}=P \cup\{f\}$. Suppose there exists an element $y \in B \backslash G f$. Since $G f$ is closed, $G f$ is a two-sided ideal so $y f \in G f$. Since $P^{-} y=P y \cup\{y f\}, P^{-} y$ is a half-ray through $y$ to a point in $G f$. This would again yield a closed triod in the boundary of $G e$. We conclude there is no such $y$ so $G f=B$.

Continue to assume $G f=f G$ is a group in $B$, but now assume $G f$ has a two-sided zero 0 in its closure. If there exists an idempotent $h \in B \backslash(G f)^{-}$then $G h=h G$, $G h$ is a group and $(G h)^{-}=(G h) \cup\{0\}$. There is certainly no other idempotent in $B$. Thus 0 is a right zero in the semigroup $(\mathrm{Ge})^{-}$which is not a limit point of idempotents (in $\left.(\mathrm{Ge})^{-}\right)$. Thus by the lemma, there is a one-parameter subgroup $P$ of $G e$ such that $P^{-}=P \cup\{0\}$. It is evident once again that under these conditions $B=(G f)^{-} \cup(G h)^{-}$. 
If there are no idempotents other than $f$ and 0 we can still use the lemma to find a one-parameter subgroup $P$ of $G e$ such that $P^{-}=P \cup\{0\}$ and this, as before, shows that $B$ is at most a closed line. If there are no nonzero idempotents in $B$ then, since $B$ certainly contains an idempotent, $B$ contains a two-sided zero which is not a limit point of idempotents in $(\mathrm{Ge})^{-}$. Once again we see from the above arguments that $B$ is the union of at most two closed half-rays from 0 .

We show finally that if $B$ is a half-ray then the endpoint of $B$ is a two-sided zero 0 for $S$ and $B^{2}=\{0\}$. It is clear that the endpoint of $B$ is a two-sided zero and that $B \backslash\{0\}$ is both a left and right orbit of $G$. If $B^{2} \neq\{0\}$ then $B \backslash\{0\}$ is a group. Let $f$ denote the identity of $B$. Then $G_{l}(f)^{-}$is a semigroup on a half-plane with two-sided zero $f$. Furthermore, $G_{l}(f)^{-} \cap B=\{f\}$ and $\left(G_{l}(f)^{-} \cap L\right) \mid\{f\}$ has two components such that either both are groups or at least one of them $A$ has the property that $A^{2}=\{f\}$. Since $L \backslash B=G e$ and $G e$ is a semigroup, the latter cannot occur. But then $A$ is a one-parameter subgroup of $G e$. If $G e=e G$, then $G e$ is a group and therefore has only one idempotent which is false since each component of $\left(G_{l}(f)^{-} \cap L\right) \backslash\{f\}$ contains an idempotent. Hence, $\operatorname{dim} e G=1, e G \subset G e$ and all one-parameter subgroups of $G e$ are conjugate to $e G$. However, there is one one-parameter subgroup of $G e$ having 0 in its closure so each one-parameter subgroup of $G e$ has 0 in its closure. It follows that $B$ has no identity so $B^{2}=\{0\}$ and the proof of the theorem is complete.

THEOREM 2.11. Suppose $e$ is an idempotent in L such that $\operatorname{dim} G e=0$ and $\operatorname{dim} e G$ $=1$. Then $L \mid e G$ is the union of two right orbits of $G$.

Proof. We have seen that under these conditions, $e G$ is a closed line in $L$ so $L \backslash e G$ is the union of two components. Let $H$ be the right isotropy group of $e$. Let $T$ denote the boundary of $H$. Thus $H^{-}=H \cup T$ is a semigroup on a half-plane. Furthermore, as a consequence of the left-right dual of Corollary 2.3.1, $T$ has points on both sides of $e G$. Let $C$ denote one of the components of $T \backslash\{e\}$. For any $y \in C, y H=C$; this is so because $e$ is a two-sided zero for $H$ and $H^{-}$is a semigroup on a half-plane. Let $P$ be a one-parameter subgroup of $G$ such that $e G=e P$. Since $e G \cap T=\{e\}, e G$ and $T$ are, in a loose sense "orthogonal". Hence, if $y \in C$ is sufficiently close to $e, y P \subset C$. Hence, for such $y, y G$ is not a line since $y H \cup y P \subset y G$. Therefore, $\operatorname{dim} y G=2$.

We claim that the only boundary points of $y G$ belong to $e G$. For assume that there exists an element $z$ in the boundary of $y G$ but not in $e G$. Certainly $\operatorname{dim} z G \neq 2$. If $\operatorname{dim} z G=0$ then $z G=z$. But then $z$ is a left zero for $S$ and $e$ is a right zero for $S$ and $z \neq e$ which is impossible. It follows that $\operatorname{dim} z G=1$ so $z G$ is a line. Let $R$ be a one-parameter subgroup of $H$ such that $R^{-}=R \cup\{e\}$. Now $e \neq z e \in z R^{-}$and $z=e$. Thus $z R^{-}$is a half-ray from $e$ through $z$. Since $z R \subset z G$ and $\operatorname{dim} z G=1, z R=z G$. Let $A$ denote the component of $L \backslash e G$ which contains $C$. Then $A \backslash z R$ is the union of two components. Let $E$ denote the component which contains $C$ and let $F$ denote the remaining component. If $y \in C$ then $y G \subset E$ since $y G \cap z R=\varnothing$. However, 
$e \in(y G)^{-}$so $e G \subset(y G)^{-} \subset E^{-}$. But it is impossible for points of $e G$ which belong to the boundary of $F$ to belong to $E^{-}$. We conclude that if $y \in C$ then $(y G)^{-}=$ $(y G) \cup(e G)$. A similar argument is applicable to the remaining component of $L \backslash(e G)$ so each component of $L \backslash e G$ is a right orbit of $G$.

In the following theorem, we consider in more detail the situation in which $e$ is an idempotent such that $\operatorname{dim} e G=0$ and $\operatorname{dim} G e=1$. It quickly develops that there are several ways in which this situation can arise so the statement of the theorem and its proof are rather long. Primarily to spare the reader, we have restricted ourselves to determining the possibilities relative to $\operatorname{dim} G w, w \in L \mid e G$ and to determining right and left isotropy groups of all elements of $L$. It would not be difficult, if one's interest did not flag, to determine at least the orbits of $G$ in which various products lie and to a large extent determine multiplication in $L$ completely in each case. We postpone this determination for the present. Also we leave until another time the proof that certain possibilities can actually occur. Thus we say, for example, that apparently a certain pair of left isotropy groups may be identical or distinct, etc. This means we have discovered no inconsistencies in the possibilities and are strongly of the opinion that they can occur but have not actually constructed examples in which they do occur.

THEOREM 2.12. Suppose $e$ is an idempotent in $L$ such that $\operatorname{dim} G e=0$ and $\operatorname{dim} e G=1$. Let $H$ be the right isotropy subgroup of $G$ at $e$. (Thus $e$ is a two-sided zero for $H$.) Then $H$ is a nonnormal planar subgroup of $G$. If $H$ is commutative then $G$ is isomorphic to $R \times \operatorname{Af}(1)$. If $H$ is not commutative then $G$ is isomorphic to the group

$$
\left(\begin{array}{ccc}
u & u \ln u & x \\
0 & u & y \\
0 & 0 & 1
\end{array}\right), \quad u>0
$$

of $3 \times 3$ matrices of real numbers.

Let the two components of $L \mid e G$ be denoted $E$ and $F$ respectively. Let $T$ denote the boundary of $H$. Let $C=E \cap T$ and let $D=F \cap T$. The possibilities for $S$ may be more particularly limited as follows:

(1) At most one of the components $E, F$ can contain an element $z$ such that $\operatorname{dim} G z=2$. If $z$ is such an element then $G z$ coincides with the component containing $z, G z$ is a group isomorphic to $\mathrm{Af}(1)$ and $G$ is isomorphic to $R \times \operatorname{Af}(1)$. Let $z$ be chosen to be the identity of $G z$. Then $G$ commutes elementwise with $z$ and if $Z$ denotes the center of $G$ then $G_{l}(z)=G_{r}(z)=Z$. Thus for all $w \in G z, G_{l}(w)=Z$. Suppose $E$ is the component containing $z$. The one-parameter subgroups of $G z$ are the conjugates of $C$ together with the set $N$ of points in L lying in the closure of the normal commutative planar group $H_{0}$ of $G$. The set $D$ may be either a group or satisfy $D^{2}=\{e\}$. The left isotropy group of any member of $D$ (and therefore $F$ ) is a noncommutative planar group. 
(2) $\operatorname{dim} G z=1$ for all $z \in L \mid e G$ and $C$ and $D$ are both groups. In this case, $H$ is commutative, so $G$ is isomorphic to $R \times \operatorname{Af}(1)$. Furthermore, there exist distinct noncommutative planar groups $K_{1}, K_{2}$ of $G$ such that $K_{1}$ is the left isotropy subgroup of any element of $E$ while $K_{2}$ is the left isotropy subgroup of any element of $F$.

(3) $\operatorname{dim} G z=1$ for all $z \in L \mid e G, C$ is a group and $D^{2}=\{e\}$. In this case $H$ is commutative and $G$ is isomorphic to $R \times \operatorname{Af}(1)$. The left isotropy subgroup of any element of $E$ is a fixed noncommutative planar group in $G$. The left isotropy subgroup of any element of $F$ is a fixed planar subgroup of $G$ distinct from $K$.

(4) $\operatorname{dim} G x=1$ for all $z \in L \mid e G, C^{2}=D^{2}=\{e\}$.

If $H$ is commutative, then $G=R \times \operatorname{Af}(1)$, there exist planar subgroups $K_{1}$ and $K_{2}$ of $G$ such that $K_{1}$ is the left isotropy subgroup of each element of $E$ while $K_{2}$ is the left isotropy subgroup of each element of $F$. Apparently $K_{1}$ and $K_{2}$ may coincide or be distinct and may be, independently of each other, noncommutative or be the normal commutative planar subgroup of $G$. If $H$ is not commutative then, of course, $G$ is isomorphic to the second of the two groups listed above and the left isotropy subgroup of each element of $L \mid e G$ is the normal, commutative planar subgroup of $G$ (that is, the commutator subgroup of $G$ ).

(5) In any case, if $z \in E$ is an element such that $\operatorname{dim} G z=2$ than $E$ is filled by mutually disjoint conjugates of $C$. The right isotropy subgroup of an element $w \in E$ is then determined as follows: w belongs to a conjugate $\left(H^{\prime}\right)^{-} \cap E$ of $\left(H^{-}\right) \cap E$. Then $G_{r}(w)=G_{l}(w) \cap H^{\prime}$.

Proof. We already know that $H^{-}$is a semigroup on a half-plane with $e \in H^{-}$. Also if $g \in G, g^{-1} e g \in\left(g^{-1} H g\right)^{-}$so, since $g^{-1} e=e, e g \in\left(g^{-1} H g\right)^{-}$. Since $H^{-} \cap e G$ $=\{e\}$, and since there exists $g \in G$ such that $e g \neq e, H$ is not normal.

Note that if $c$ is any central element of $G$ then $c \in H$, since $g e=e$ for all $g \in G$ and $e c=c e$. Already then, we can say that $G$ is not nilpotent (else $H$ would be normal) or simple, since the center of $\mathrm{Sl}(2)$ is contained in no planar subgroup. Therefore, $G$ is either isomorphic to $R \times \operatorname{Af}(1)$ or $G$ is isomorphic to one of the semidirect products $R \cdot V_{2}$ where $V_{2}$ denotes the two-dimensional vector group.

Suppose $H$ is commutative. Then $G$ is not isomorphic to one of the groups $R \cdot V_{2}$. For suppose it is, then the commutator subgroup $V$ of $G$ is isomorphic to $V_{2}$. Since $H$ is not normal, $H \neq V$ so $H \cap V$ is a one-parameter subgroup of $G$. Since $H \cap V$ commutes elementwise with $H$ and with $V$, it commutes elementwise with $G$. That is, $G$ has a nontrivial center. But an examination of the Lie algebra of any one of the groups $R \cdot V_{2}$ reveals that no such group has a nontrivial center (the assumption of a center leads to the contradiction that $\left.\operatorname{dim} L^{\prime}=1\right)$. Therefore, if $H$ is commutative then $G$ is isomorphic to $R \times \operatorname{Af}(1)$.

Now suppose $H$ is not commutative. Therefore $G$ is not isomorphic to $R \times \operatorname{Af}(1)$ since the latter group has a nontrivial center while $H$ does not. Hence $G$ is isomorphic to one of the semidirect products $R \cdot V_{2}$. Furthermore, since $G$ contains the noncommutative planar group $H$, the following situation must obtain for the 
Lie algebra $\mathscr{L}$ of $G$ : Let $\mathscr{L}^{\prime}$ denote the derived subalgebra and let $g \in \mathscr{L} \backslash \mathscr{L}^{\prime}$. Then ad $g$ acting in $\mathscr{L}^{\prime}$ has an invariant subspace (corresponding to the normal subgroup $V \cap H)$.

In order to determine $G$ yet more exactly, we must utilize information about $H^{-}$not used up to now. Since $H^{-}$is a semigroup on a half-plane with two-sided zero $e$ and since $H$ is not commutative, it follows that every nonnormal oneparameter subgroup of $H$ has $e$ in its closure. For 0 is not a limit point (in $\mathrm{H}^{-}$) of idempotents, yet every point of $H$ belongs to a one-parameter subgroup. Thus, there is at least one nonnormal such group. All others are conjugate to this one, so the assertion follows.

As a consequence of this fact, there can be no noncommutative planar subgroup of $G$ which is not conjugate to $H$. For suppose $K$ is such a group. Then $K \cap H$ is a nonnormal one-parameter subgroup of $H$. For if $K \cap H$ is normal in $H$ then $K \cap H=H \cap V=K \cap V$. Now it is not difficult to show that two noncommutative planar subgroups of a group $R \cdot V_{2}$ are conjugate if and only if they intersect $V$ in the same one-parameter subgroup. Therefore $e \in K^{-}$, so $K^{-}$is a semigroup on a half-plane with $e \in K^{-}$. Since $K \neq H$ and $e G \neq e, e K \neq e$, so $e$ is not a two-sided zero for $K^{-}$. Hence $K^{-}$is isomorphic to Af $(1)^{-}$. Therefore, if $Q$ is the normal oneparameter subgroup of $K$ then $e Q=e$ (since $K e=e$ and $\operatorname{dim} e K=1)$. Now $Q=K \cap V$. Since $K$ is not conjugate to $H, K \cap V$ and $H \cap V$ are distinct one-parameter subgroups of $V$. Since $e(K \cap V)=e$ and $e(H \cap V)=e$, it follows that $e V=e$. But since $e H=e$ and $V \neq H$, this implies $e G=e$ which is a contradiction.

$\mathrm{Up}$ to this point we have shown that if $H$ is not commutative then every noncommutative planar subgroup is conjugate to $H$. An examination of the Lie algebras of the groups $R \cdot V_{2}$ reveals that there is exactly one which has precisely one conjugacy class of noncommutative planar subgroups. This group is the one listed in the statement of the theorem so that part of the theorem is proved.

We prove next that if $\operatorname{dim} G z=2$ for some $z \in C$ then $G z$ is a group. For, recalling that $C=H z=H^{-} \cap E=T \cap E$, we know that either $C^{2}=\{e\}$ or $C$ is a group. We also know, in any case, that if $\operatorname{dim} G z=\operatorname{dim} z G=2$ then $G z=z G$. Suppose $C^{2}=\{e\}$. It follows that $x y=e$ for all $x, y \in G z$. But then $x y=e$ for all $x, y \in(G z)^{-}$. However, if $e g \neq e$ then $(e g)^{2}=(e g),(e g)=e g \neq e$, which is a contradiction.

We conclude that if there exists an element $z \in T \cap E$ such that $\operatorname{dim} G z=2$ then $C$ is a grol'p. Therefore we may assume $z^{2}=z$. Hence, as we have seen, $G z=z G$ is a group. We can, in fact, say somewhat more. The one-parameter subgroup $Q$ of $H$ such that $Q^{-}=Q \cup\{z\}$ is normal in $G$ and $G$ commutes elementwise with $z$. Also $H$ cannot be the noncommutative planar group, for then every element of $T$ is an idempotent (which is impossible in this case) or $e$ is a two-sided zero for $\mathrm{H}^{-}$ and $T^{2}=\{e\}$ (which is also impossible if $C$ is a group). Thus, $H$ is commutative and $G$ is isomorphic to $R \times \operatorname{Af}(1)$. Since the only normal one-parameter subgroup of a commutative planar group in $R \times \operatorname{Af}(1)$ is the center $Q_{0}$ of $G$, we have $Q_{0}=Q$ so the normal one-parameter subgroup of $G$ which runs to $z$ is the center of $G$. This 
implies that $G z$ is isomorphic to $G / Q_{0}$ and hence to Af (1). It also implies that the other component $F$ of $L \backslash e G$ can contain no element having a two-dimensional left orbit since surely the center of $G$ cannot run to more than one element of $L$.

The remaining conclusions about case (1) are now easy to draw. Clearly, $G_{l}(w)=$ $G_{r}(w)=Q_{0}$ for every $w \in G z$. Also, $C$ is a one-parameter subgroup of $G z$ and all its conjugates are such. Multiplication in $e G$ makes it clear that $(G z)^{-}$is isomorphic to Af $(1)^{-}$. The normal one-parameter subgroup $N$ of $G z$ cannot, of course be conjugate to $C$. On the other hand, since $Q_{0}^{-}=Q_{0} \cup\{z\}$ and since the normal commutative subgroup $H_{0}$ of $G$ is generated by $Q_{0}$ and the normal one-parameter subgroup $P_{0}$ of the various noncommutative planar groups of $G, z \in H_{0}^{-}$. Also $H_{0} z=P_{0} Q_{0} z=P_{0} z=z P_{0}=z H_{0}$. Since $\operatorname{dim} G z=2, H_{0} z \neq z$ so $H_{0} z$ is a one-parameter subgroup of $G z$. Since $H_{0}$ is normal and $z$ is central, $H_{0} z$ is the normal oneparameter subgroup of $G z$, whence $H_{0} z=N$. To see that $N$ forms the complete boundary of $H_{0}$ in $S$, first let $x \in H_{0}^{-} \cap E$. Then $x=g z$ for some $g \in G$. Let $p_{n} \in P_{0}, q_{n} \in Q_{0}$ be such that $p_{n} q_{n} \rightarrow g z$. Then $p_{n} q_{n} z \rightarrow g z$ so $p_{n} z \rightarrow g z$. But $H_{0} z$ is closed in $(G z)^{-}$and hence in $S$ so since $p_{n} z \in H_{0} z, g z \in H_{0} z$. Since $H_{0}^{-} \backslash H_{0}$ is an ideal in $H_{0}^{-}$, it is connected. Thus $H_{0}^{-} \cap L=H_{0}^{-} \cap E=H_{0} z$. Except for giving examples that $D$ may be either a group or $D^{2}=\{e\}$, all of the assertions in (1) have been proved.

Now suppose $\operatorname{dim} G z=1$ for one and hence every $z \in E \cap T$. Since $G z$ is a line and $H z$ is a line such that $(H z)^{-}=H z \cup\{e\}, G z=H z=T \cap E$. Let $x \in E$. Then there exists $g \in G$ such that $x=z g$. Since $z \in T \cap E, g^{-1} x g \in g^{-1}(T \cap E) g$ so $g^{-1} x \in g^{-1}(T \cap E) g$. Now $G\left(g^{-1}(T \cap E) g\right)=g^{-1}(G(T \cap E)) g=g^{-1}(T \cap E) g$. In particular, $G \cdot g^{-1} x \subset g^{-1}(T \cap E) g$. Therefore $x \in g^{-1}(T \cap E) g$ and it follows that $E$ is covered by conjugates of $T \cap E$ and that $\operatorname{dim} G w=1$ for all $w \in E$.

We claim that distinct conjugates of $T \cap E$ are disjoint. For assume

$$
\left(g^{-1}(T \cap E) g\right) \cap(T \cap E) \neq \varnothing .
$$

Thus suppose $g^{-1} z g=w$ for some $z, w \in T \cap E$. Then $G w=G g^{-1} z g=G z g$. Since $\operatorname{dim} G z=\operatorname{dim} G w=1, G z=T \cap E=G w$. Hence $T \cap E=(T \cap E) g$. Now $e \in(T \cap E)^{-}$ so $e g \in(T \cap E)^{-} g=((T \cap E) g)^{-}=(T \cap E)^{-}$. But $(T \cap E)^{-} \cap(e G)=\{e\}$ so $e g=e$, whence $g \in H$ and $g^{-1}(T \cap E) g=T \cap E$.

Since the same argument applies if $z \in F$, we have shown that if $z \in L \mid e G$ is such that $\operatorname{dim} G z=1$ then the component of $L \mid e G$ containing $z$ is covered by mutually disjoint conjugates of $\mathrm{H}^{-} \cap L$ lying in that component.

We notice that the left isotropy group of any two elements belonging to a given component of $L \mid e G$ are equal while if the left isotropy group $K$ of the elements in one of the components of $L \mid e G$-say $E$-has the property that $K^{-} \cap E \neq \varnothing$ then $K$ must be distinct from the left isotropy group of the elements of $F$. Indeed, any two elements belonging to a given right orbit of $G$ generally have the same left isotropy group. To see the truth of the second assertion notice that no element of $E^{-}$can fix any element of $F$ on the left-that is, if $v \in E^{-}$and $w \in F$ then 
$v w \neq w$. For clearly $E^{-}$is an ideal in $S$ so $v w \in E^{-}$while $w \notin E^{-}$. Thus if $K$ is the left isotropy group of the elements of $E$ and $v \in K^{-} \cap E$ then $K$ cannot be the left isotropy group of the elements of $F$ since then $v w=w$ if $w \in F$.

It follows from the last assertions that if $\operatorname{dim} G z=2$ for some $z \in E$ then the left isotropy group of the elements of $F$ is a noncommutative planar group. For, as we show in the next paragraph, $G_{l}(w)$ is not conjugate to $H$ for any $w \in L \mid e G$ under any condition. The only other possibility is that it be the commutative normal planar group $H_{0}$. But when $E=G z=z G, H_{0}^{-} \cap E \neq \varnothing$ since, as we have seen, the center $Z$ runs to the identity of $E$. This completes the proof of all assertions made in case (1).

To gain information about the various right isotropy groups of elements in $L \mid e G$, we make the general observation that if $x \notin e G$ then $H x=x$. For, since $e G$ is closed, it is a right ideal. Therefore $e x \in e G$ and hence $e x \neq x$. If $H x=x$ then $H^{-} x=x$ so $e x=x$. It follows from this that $G_{l}(w)$ is not conjugate to $H$ for any $w \in L \mid e G$. For suppose $w \in L \mid e G$ and $G_{l}(w)=g^{-1} H g$. That is, $g^{-1} H g \cdot w=w$. So $H g w=g w$. But if $w \notin e G$ then $g w \notin e G$ since then $w \in g^{-1} e G=e G$.

It follows that if $w \in H^{-}$then $G_{r}(w)=G_{l}(w) \cap H$. For let $Q$ be the one-parameter subgroup of $H$ such that $Q w=w$. If $H$ is commutative, then $Q w=w Q$ so $G_{r}(w)=Q$. Since $G_{l}(w) \neq H, Q=G_{l}(w) \cap H$. If $H$ is not commutative then the subgroup $Q$ of $H$ such that $Q w=w$ is the normal one-parameter subgroup of $H$ and being the only closed subgroup of $H^{-}$also satisfies: $w Q=w$. Thus $Q \subset G_{l}(w)$ so $G_{r}(w)=Q$. As before, $Q=G_{l}(w) \cap H$.

If $w$ belongs to a conjugate $\left(H^{\prime}\right)^{-}$of $H^{-}$then $G_{r}(w)=G_{l}(w) \cap H^{\prime}$. For let $w \in\left(H^{\prime}\right)^{-} \cap L$ with $H^{\prime}=g^{-1} H g$. Then $H^{\prime}$ is the right isotropy subgroup of eg. The argument just completed is applicable here to yiel 1 the indicated result, where $e g$ plays the role just played by $e$.

The right isotropy groups of all elements of $L$ have been identified in all cases, at least in terms of the corresponding left isotropy groups.

It remains to identify, as far as possible, the various left isotropy groups and the various possibilities for multiplication on $C$ and $D$.

Let $z \in L \mid e G$. If $\operatorname{dim} G z=2$ we have already seen that $G_{l}(z)$ is the center of $G$. Suppose $\operatorname{dim} G z=1$. Let $K=G_{l}(z)$. Suppose $G$ is isomorphic to the second of the two groups listed in the statement of the theorem. Since $K$ is not conjugate to $H$, $K$ is the commutator subgroup of $G$. If, instead, $G$ is isomorphic to $R \times \operatorname{Af}(1)$ and the component of $L \mid e G$ not containing $z$ is a group we have seen that $G_{l}(z)$ is a noncommutative planar group.

If $\operatorname{dim} G z=1$ and $z^{2}=z$ then $K$ is one of the noncommutative groups of $G$. For, to be definite, suppose $z \in F$. Then we may suppose $z \in H^{-}$since in any case $z$ belongs to a conjugate of $H^{-}$. Let $Q$ be the one-parameter subgroup of $H$ such that $Q^{-}=Q \cup\{z\}$. Since the various conjugates of $D$ in $F$ are, under the present circumstances, mutually disjoint, $Q$ is not normal in $G$. If $G_{l}(z)$ is the normal commutative planar group $H_{0}$ then $Q=H \cap H_{0}$. But $H \cap H_{0}$ is central and 
therefore normal which is a contradiction. Incidentally, we see in this case that $K^{-} \cap F \neq \varnothing$. Since the left isotropy group of all elements of either component of $L \mid e G$ are the same we conclude that if a given component of $L \backslash e G$ contains an idempotent but is not a group then the corresponding left isotropy group is a noncommutative planar group having points of that component in its closure. It follows that if both $E$ and $F$ contain idempotents but neither are groups then the isotropy groups for the elements of the two components must be distinct.

The possible multiplications on $C$ and $D$ are of course known. For $H^{-}$is a semigroup with zero on a half-plane. Thus either $C$ and $D$ are both groups, or one, say $E$ is a group and $D^{2}=\{e\}$, or $C^{2}=D^{2}=\{e\}$.

We have not determined the left isotropy group of an element of $C$ if $C^{2}=\{e\}$ and $H$ is commutative. We shall settle this matter later. Apparently it may be either a noncommutative planar group or the normal commutative planar group. Also, apparently, it is not influenced by the left isotropy group of elements of $D$ if $D$ is a group.

TheOREM 2.13. Suppose $e^{2}=e \in L$ is such that $\operatorname{dim} G e=2$ and $\operatorname{dim} e G=0$. Then $G$ is a nonhyperbolic semidirect product $R \cdot V_{2}$. If, conversely, $G$ is a nonhyperbolic semidirect product then $G$ is the maximal group of a semigroup on a half-space containing an idempotent e such that $\operatorname{dim} G e=2$ and $\operatorname{dim} e G=0$. Of course, if $L$ is the boundary of such a semigroup, $L$ is left trivial; i.e. $x y=x$ for all $x, y \in L$.

Proof. Since $\operatorname{dim} e G=0, G e=L$ by Theorem 2.7. Since $\operatorname{dim} G e=2, G_{l}(e)$ is a one-parameter subgroup $P$ of $G$ such that $P^{-}=P \cup\{e\}$. For any $g \in G, e g^{-1}=e$ so $\left(g P g^{-1}\right)^{-}=\left(g P g^{-1}\right) \cup\{g e\}$. Since $G e=L$, this implies $P$ is not normal.

Since $e G=e$, the center $C$ of $G$ is contained in $P$. But since $P$ is not normal, $P$ is not central. This means that $C$ is either trivial or infinite cyclic and the latter holds only if $G$ is isomorphic to $\mathrm{Sl}$ (2). In particular, $G$ is not nilpotent nor is $G$ isomorphic to $R \times \mathrm{Af}$ (1) since nilpotent groups and $R \times \mathrm{Af}(1)$ have nontrivial centers. In fact, $G$ is not isomorphic to $\mathrm{Sl}(2)$ either. For then $e \in(C)^{-}$so for every $g \in G$, $g e \in\left(g C g^{-1}\right)^{-}=C^{-}$which is impossible since $P^{-}=P \cup\{e\}$. The only possibility for $G$ is one of the semidirect product groups $R \cdot V_{2}$.

To see that $G$ cannot be one of the hyperbolic groups, note that if it is, then there exist two normal one-parameter subgroups $Q_{1}$ and $Q_{2}$ of the commutative subgroup $V$ of $G$. Thus $P Q_{1}$ and $P Q_{2}$ are two noncommutative planar subgroups $K_{1}$ and $K_{2}$ of $G$. Of course, $e \in K_{1}^{-}$and $e \in K_{2}^{-}$.

We prove $K_{1}^{-}=K_{1} \cup K_{1}$ e. First, $Q_{1}$ must operate on $L$ on the left without fixed points since the left isotropy groups of the various points in $L$ are conjugates of $P$ which $Q_{1}$ is not. Thus every left orbit of $Q_{1}$ in $L$ is a closed line by [3]. In particular $Q_{1} e$ is closed. Let $x \in K_{1}^{-} \cap L$. Then $x=g e$ for some $g \in G$ and $x=\lim q_{n} p_{n}$ for some sequence $\left\{q_{n} p_{n}\right\}_{n}$ with $q_{n} \in Q_{1}, p_{n} \in P$. Therefore $x=x e=\lim q_{n} p_{n} e=\lim q_{n} e$ since $P e=e P=e$. But $q_{n} e \in Q_{1} e$ so $x \in Q_{1} e=K_{1} e$. 
It now follows by the result in the appendix that $K_{1}^{-}$is a semigroup on a halfplane. Since all elements of $K_{1}^{-} \cap L$ are idempotent, $K_{1}^{-}$is isomorphic to Af (1)by [4]. (Af (1) ${ }^{-}$is the closure of $\mathrm{Af}(1)$ in the representation of $\mathrm{Af}(1)$ given above.)

What has just been proved about $K_{1}$ and $K_{1}^{-}$is equally true about $K_{2}$ and $K_{2}^{-}$ respectively. Thus the argument which was used in the proof of Theorem 2.5 to show that $G$ is not hyperbolic is available here so the proof of the first half of the theorem is complete.

We observed (parenthetically) in the discussion that preceded Theorem 2.5 that each nonhyperbolic semidirect product $R \cdot V_{2}$ has a representation as a group of $3 \times 3$ matrices in such a way that its closure in the semigroup of all $3 \times 3$ matrices is a semigroup on a half-space containing an idempotent $e$ with $\operatorname{dim} G e=2$ and $\operatorname{dim} e G=0$. Thus the proof of the theorem is complete.

THEOREM 2.14. Let $P$ be a one-parameter subgroup of $G$ which is not closed in $S$. Then there exists an idempotent e such that $P^{-}=P \cup\{e\}$.

Proof. We already know that there exists an idempotent $e \in P^{-}$. By [4], it is sufficient to prove $P e=e$. Also, because of the known structures of semigroups on a half-plane, the conclusion follows if at anytime it is known that $P$ is contained in a half-plane semigroup.

Assume $P e \neq e$. Then $e P \neq e$ so $G e \neq e$ and $e G \neq e$. Suppose first that $\operatorname{dim} G e=1$. Let $H=G_{l}(e)$. If $P$ has a conjugate in $H$ then $P$ is contained in a half-plane semigroup. If $P$ has no conjugates in $H$ then $G e=P e$ and $H^{-}$is a local cross-section to the orbits of $P$ at $e$. Then $P e$ has points on both sides of $H^{-}$. Since $P e \subset P^{-}, P$ must intersect $H$ repeatedly which is impossible for any group in $E_{3}$. A similar argument applies if $\operatorname{dim} e G=1$.

If $\operatorname{dim} G e=\operatorname{dim} e G=2$ then $G_{l}(e)$ is a normal one-parameter group $Q$. Since $P e \neq e, P e$ is a one-parameter subgroup of $G e$ lying in the boundary of the planar group $P Q$ and closed in $G e$. As we have seen before, $P e$ forms the entire boundary of $P Q$ in $G e$ so by the result in the appendix $P Q \cup P e$ is a semigroup on a halfplane with $e \in P^{-}$and the conclusion follows in this case also.

3. Semigroups with $G$ isomorphic to $\mathrm{Sl}(2)$. Examples have been or will be offered which show that every group in $E_{3}$, with the possible exception of $\mathrm{Sl}(2)$, can be the maximal group of a semigroup on a half-space. We can not yet offer an example in which $G$ is isomorphic to $\mathrm{Sl}(2)$, nor can we yet rule out the existence of such an example. In this section we show that if there is a semigroup on a half-space with $G \cong \mathrm{Sl}(2)$ then it must be essentially unique.

Thus, throughout this section we assume that $S$ is a semigroup on a half-space and that its maximal group $G$ is isomorphic to the universal covering group Sl (2) of the group sl (2) of $2 \times 2$ real matrices of determinant 1 .

It is useful to have a number of facts about $\mathrm{Sl}(2)$ readily available. Let $\gamma$ denote the natural or covering homomorphism from $\mathrm{Sl}$ (2) to sl (2). The kernel $Z$ of $\gamma$ is 
an infinite cyclic central subgroup of index 2 in the center $C$ of Sl (2), the center of sl (2) consisting of the two element group. The compact one-parameter subgroups of sl (2) are all conjugate to the group of rotations in the plane. If a one-parameter subgroup $P$ of $\mathrm{Sl}$ (2), hereafter simply denoted by $G$, is mapped onto a compact one-parameter group by $\gamma$ we shall call $P$ distinguished. This condition is evidently equivalent to containing the center $C$. Certainly if $P \supset C$ then $\gamma(P)$ is compact. If $\gamma(P)$ is compact then $P \cap Z$ is a nontrivial, cyclic subgroup of $P$. Let $c_{0}$ be a generator of $C$. Let $c_{0}^{k}$ be a generator of $P \cap Z$. Let $c_{1}$ be the $k$ th root in $P$ of $c_{0}^{k}$. Since $c_{0}$ is central, $c_{0} / c_{1}$ is well defined and if $C \notin P, c_{0} / c_{1} \neq 1$. However, $\left(c_{0} / c_{1}\right)^{k}$ $=c_{0}^{k} / c_{1}=1$ so $G$ contains an element of finite order which is impossible for a group on a Euclidean space. Thus every distinguished one-parameter subgroup of $G$ contains the center.

Next note that the only elements of Sl (2) which fail to lie on some one-parameter subgroup belong to a set $c H$ for some nontrivial central element $c$ and some planar subgroup $H$ of $G$. To see this, suppose $A$ is a $2 \times 2$ real matrix of determinant 1 . If $A$ is irreducible then $A$ is conjugate to a rotation and hence lies on a one-parameter subgroup of sl (2). If $A$ is reducible then $A$ is conjugate (in sl (2)) to a matrix of the form

$$
\left(\begin{array}{cc}
t & b \\
0 & 1 / t
\end{array}\right)
$$

Now the group of all such matrices with $t>0$ is a (noncommutative) planar group, all of whose elements lie on one-parameter subgroups. If $t<0$ then $A$ can lie on no one-parameter group. However, in that case,

$$
\left(\begin{array}{cc}
t & b \\
0 & 1 / t
\end{array}\right)=z_{0}\left(\begin{array}{rc}
-t & b \\
0 & -1 / t
\end{array}\right)
$$

where $z_{0}$ is the nontrivial central element of sl (2). Thus $A \in z_{0} H$ where $H$ is a planar group. Suppose $x \in G$ lies in no one-parameter subgroup of $G$. If $\gamma(x)$ lies on a compact one-parameter subgroup of sl (2) then $\gamma(x) \in \gamma(P)$ for some distinguished one-parameter subgroup $P$ of $G$. But then $x \in P z=P$. If $\gamma(x)$ belongs to a planar group $H$ then $x \in H^{\prime} x$ where $H^{\prime}$ is a planar group in $G$ so $x \in c H^{\prime}$ for some central element $c$ and $H^{\prime}$ is a planar group. If $\gamma(x)$ belongs to no one-parameter group then $\gamma(x) \in z_{0} H$ so $x \in C z_{0} H^{\prime} \subset C H^{\prime}$ where $H^{\prime}$ is a planar group.

The planar groups of $G$ are all isomorphic to Af (1) and are conjugate to each other. Let $H$ be a planar subgroup of $G$ and let $Q$ be the normal (in $H$ ) oneparameter subgroup. The set of all conjugates of $Q$ form a (topological) cone $\mathscr{C}$ in $G$ which plays a significant role in determining the structure of $S$. The various claims made about $\mathscr{C}$ can perhaps best be established by recalling properties of the Lie algebra $\mathscr{L}$ of $G$. The algebra $\mathscr{L}$ is isomorphic to the Lie algebra on the set of $2 \times 2$ matrices of the form:

$$
X=\left(\begin{array}{rr}
x & y \\
z & -x
\end{array}\right), \quad x, y, z \text { real. }
$$


The element $X$ belongs to the ideal of a two-dimensional subalgebra of $\mathscr{L}$ if and only if ad $X$ is nilpotent. If $X$ is nilpotent then $X$ is nilpotent and in this case conversely. But if $X$ is nilpotent then $x^{2}=-y z$. Thus, the elements of $\mathscr{L}$ which belong to ideals of two-dimensional algebra form the cone $x^{2}=-y z$. If $x^{2}=-y z$ then $\exp X=I+X$ where $I$ is the identity matrix. Hence the exponential map is a homeomorphism on the cone $x^{2}=-y z$. It follows that $\mathscr{C}$ is a cone. Furthermore, the points belonging to distinguished one-parameter groups lie "inside" $\mathscr{C}$ while the points on planar groups lie on or "outside" $\mathscr{C}$.

We need one further fact about $G$. By definition, any two one-parameter groups contained in $\mathscr{C}$ are conjugate. It is important to note, however, that a complete set of conjugates $p Q p^{-1}$ of a given $Q$ in $\mathscr{C}$ is gotten by restricting $p$ to lie in the compact interval $\left[1, c_{0}\right]$ of a distinguished one-parameter subgroup of $G$, (here, $c_{0}$ denotes a generator of the center of $G$ ). This is easy to see as soon as it is realized that each element of sl (2) can be represented (uniquely) as a product of the form

with $t>0$.

$$
\left(\begin{array}{rr}
\cos \theta & \sin \theta \\
-\sin \theta & \cos \theta
\end{array}\right)\left(\begin{array}{cc}
t & b \\
0 & 1 / t
\end{array}\right)
$$

We turn now to study the structure of a topological semigroup $S$ on a half-space whose underlying group $G$ is isomorphic to $\mathrm{Sl}(2)$.

First recall the two results proved in the first section: (1) If $x \in L$ and $x G \subset G x$ then $G_{r}(x)$ is normal, and (2) if $\operatorname{dim} G x=2$ then $x G \subset G x$ so if $\operatorname{dim} G x=\operatorname{dim} x G=2$ then $G x=x G$. While these results hold for any group on $E_{3}$ they obviously have special significance now since $\mathrm{Sl}(2)$ has no normal, nondiscrete subgroup.

THEOREM 3.1. If $S$ is a semigroup on a half-space whose maximal group $G$ is isomorphic to $\mathrm{Sl}(2)$ then the boundary $L$ of $G$ contains exactly one idempotent $e$ and $e$ is a two-sided zero for $S$.

Proof. We already know that $L$ contains an idempotent. We will show, by contradiction, that if $e$ is any idempotent in $L$ then $\operatorname{dim} G e=\operatorname{dim} e G=0$. This implies that $e$ is a two-sided zero for $S$ so, since a semigroup can have at most one two-sided zero, $L$ contains a zero and no other idempotents.

We have just observed that if $\operatorname{dim} G x=2$ or $\operatorname{dim} x G=2$ then $G$ must contain a normal subgroup of dimension 1 which is false. Thus in particular, $\operatorname{dim} G e \leqq 1$ and $\operatorname{dim} e G \leqq 1$. Suppose $\operatorname{dim} G e=1$. If $\operatorname{dim} e G=1$ then $G e=e G$ by Theorem 2.5 so $G_{r}(e)$ is a normal planar group which is impossible. Thus $\operatorname{dim} e G=0$. But then $G \not \mathrm{Sl}$ (2) by Theorem 2.12. Similarly we cannot have $\operatorname{dim} e G=1$ and $\operatorname{dim} G e=0$. The only remaining possibility is $\operatorname{dim} G e=\operatorname{dim} e G=0$.

Throughout the remainder of this section we will denote the idempotent in $L$ by " 0 ".

THEOREM 3.2. If $G \cong \mathrm{Sl}(2)$ and $x \in L \mid\{0\}$ then $\operatorname{dim} G x=\operatorname{dim} x G=1$. 
Proof. The desired conclusion requires only the knowledge that $\operatorname{dim} G x \neq 0$ $\neq \operatorname{dim} x G$ since $\operatorname{dim} G x=2$ and $\operatorname{dim} x G=2$ are already ruled out. But since 0 is a two-sided zero, $0 \in(G x)^{-}$and $0 \in(x G)^{-}$for all $x \in L \mid\{0\}$.

THEOREM 3.3. Assume that $S$ is a semigroup on a half-space with $G \cong \mathrm{Sl}(2)$. If $x \in L \backslash\{0\}$ then $G_{l}(x)$ is a planar subgroup and $G x=P x$ where $P$ is any distinguished one-parameter subgroup of $G$.

Proof. By the above, $\operatorname{dim} G x=1$. But $G x$ is never a simple closed curve so $G x$ is a line and $G_{l}(x)$ is a planar subgroup. The only one-parameter subgroups having no conjugates in $G_{l}(x)$ are distinguished and each such group has this property.

THEOREM 3.4. If $G \cong \mathrm{Sl}(2)$ then 0 is not in the closure of any planar subgroup of $G$.

Proof. If 0 is in the closure of one, it is in the closure of every planar subgroup of $G$ since any two such groups are conjugate. Now 0 can not belong to the closure of the isotropy subgroup of any nonzero element. Since each $x \in L \mid\{0\}$ has $G_{l}(x)$ as a planar group, the conclusion follows.

THEOREM 3.5. Let $\mathscr{C}$ denote the cone in $G$ that is the union of the normal oneparameter subgroups of the various planar groups in $G$. Then $\mathscr{C}$ is closed in $S$.

Proof. First, observe that each one-parameter group $Q$ contained in $\mathscr{C}$ is closed in $S$. For otherwise there is an idempotent in $Q^{-} \cap L$ so $0 \in Q^{-}$and 0 belongs to the closure of a planar group in $G$.

Let $Q$ be a fixed one-parameter group contained in $\mathscr{C}$. Assume $h_{n} \rightarrow x$ for some $h_{n} \in \mathscr{C}$ and $x \in S$. For each $n$, there exists elements $p_{n}$ and $q_{n}$ with $q_{n} \in Q$ such that $h_{n}=p_{n} q_{n} p_{n}^{-1}$. Moreover, as we mentioned earlier, the $p_{n}$ may be chosen from a compact subset of a distinguished one-parameter subgroup $P$ of $G$. Thus we may suppose $p_{n} \rightarrow p$ for some $p \in P$ so $p_{n}^{-1} \rightarrow p^{-1}$. It follows that $q_{n} \rightarrow p x p^{-1}$, which implies $p x p^{-1} \in Q$ by our first observation. Thus, $x \in \mathscr{C}$ so $\mathscr{C}$ is closed.

THEOREM 3.6. If $G$ is isomorphic to $\mathrm{Sl}$ (2) and $P$ is a distinguished one-parameter subgroup of $G$ then $0 \in P^{-}$.

Proof. As above, let $\mathscr{C}$ denote the cone formed by the union of those oneparameter subgroups which are normal one-parameter groups of the various planar groups. As we mentioned above, the distinguished one-parameter subgroups of $G$ lie "inside" $\mathscr{C}$. We first want to see that 0 lies in the closure of the inside (rather than the outside) of $\mathscr{C}$. We give two proofs of this fact, one being a simple consequence of the fact that 0 belongs to the closure of no planar group, the other seeming somewhat more general.

First we note that if $c$ is a nontrivial central element and $H$ is a planar subgroup of $G$ then $c H \cap \mathscr{C}=\varnothing$. For otherwise there exists a one-parameter group $Q \subset \mathscr{C}$, an element $q \in Q$ and an element $h \in H$ such that $c h=q$. Since $c \in C, \gamma(c)$ is either the identity of $\mathrm{sl}(2)$ or $\gamma(c)$ is the nontrivial central element $z_{0}$ of sl (2). Now $\gamma(H)$ 
is a planar group in sl (2) and $\gamma(q)$ belongs to a one-parameter subgroup. Since no element of $z_{0} \cdot \gamma(H)$ belongs to a one-parameter subgroup $\gamma(c)=1$ and $\gamma(h)=\gamma(q)$. Since no two noncompact one-parameter subgroups of sl (2) intersect except trivially $\gamma(P)=\gamma(Q)$ where $P$ is the one-parameter subgroup of $H$ containing $h$. Thus, $P C=Q C$. But $P$ and $Q$ are the components of the identity in $P C$ and $Q C$ respectively so $P=Q$. It follows that $c Q \cap Q \neq \varnothing$ whence $c Q=Q$ and $c=1$, contrary to the fact that $c$ is a nontrivial central element. It follows that $c H \cap \mathscr{C}=\varnothing$ for each $c \neq 1$ in $C$ and planar group $H$.

We know, then, that every point outside $\mathscr{C}$ belongs to a one-parameter subgroup lying in some planar group. Suppose 0 belongs to the closure of the outside of $\mathscr{C}$. Then every neighborhood of 0 contains a point lying on a one-parameter subgroup of a planar group. Therefore, by Lemma 2.9 , there is a one-parameter group $P$, lying in a planar group $H$, such that $0 \in P^{-}$. But then $0 \in H^{-}$contrary to Theorem 3.4.

As an alternate proof to the preceding, assume again that 0 lies in the closure of the outside of $\mathscr{C}$. Run an $\operatorname{arc} A$ from 0 to a point of $\mathscr{C}$ other than the identity. Let $P$ be a distinguished one-parameter subgroup of $G$ and let $\left[1, c_{0}\right]$ denote the interval in $P$ from 1 to a generator of $C$. As $p$ ranges from 1 to $c_{0}$ in $\left[1, c_{0}\right]$, the endpoints of $p \cdot A p^{-1}$ describe a simple closed curve on the cone $\mathscr{C}$ and the arcs $p A p^{-1}$ must go around the outside of $\mathscr{C}$, back around to $A$. However, the arcs $p A p^{-1}$ converge pointwise and one can then show even uniformly to $A$. Since it is impossible for arcs both to go around $\mathscr{C}$ and converge uniformly to $A$, we conclude that 0 does not belong to the closure of the outside of $\mathscr{C}$.

We now prove that every neighborhood of 0 contains points lying on a distinguished one-parameter subgroup of $G$. By Lemma 2.9 again, the conclusion of the theorem follows.

Let $\mathscr{C}_{-}$denote the nape of $\mathscr{C}$ lying between 0 and the other nape of $\mathscr{C}$. Let $c_{0}$ be the generator of $C$ which is contained between $\mathscr{C}_{-}$and $L$. For each positive integer $n$, let $D_{n}$ be the component of $G \mid\left(c_{0}^{n} \mathscr{C}_{-}\right)$contained between $c_{0}^{n} \mathscr{C}_{-}$and $L$. Evidently $D_{n+1} \subset D_{n}$. Furthermore, $\bigcap_{n} D_{n}=\varnothing$. It follows that every neighborhood $V$ of 0 intersects some $c_{0}^{k} \mathscr{C}_{-}$. For if we take $V$ to be a connected neighborhood of 0 and $V \cap c_{0}^{k} \mathscr{C}_{-}=\varnothing$ then $V \subset D_{k}$. Since $V \nsubseteq \bigcap_{n} D_{n}, V \cap c_{0}^{k \mathscr{C}} \neq \varnothing$ for some $k$. Since all points between $c_{0} \mathscr{C}$ and $\mathscr{C}$ _ lie on distinguished one-parameter groups, it follows that points of $c_{0} \mathscr{C}_{-}$can be approximated arbitrarily closely by points lying on distinguished one-parameter groups. Therefore $V$ contains points lying on distinguished one-parameter groups. The proof of the theorem is complete.

We next prove a result which can be stated for any $G$ but whose most immediate application is to the case $G \cong \mathrm{Sl}(2)$.

Let $S$ then denote an arbitrary semigroup on a half-space with maximal group $G$. Let $P_{1}, P_{2}$ be commutative subgroups of $G$ and let $x \in L$. Set

$$
J\left(P_{1}, P_{2}, x\right)=\left\{p \in P_{1}: p x \in x P_{2}\right\}
$$


If $P_{1}, P_{2}$ are understood, we set $J\left(P_{1}, P_{2}, x\right)=J_{x}$ and if $x$ is also understood, we abbreviate " $J_{x}$ " to " $J$ ". Evidently $J$ is a subgroup of $P_{1}$ and for each $p \in P_{1}$, $J_{p x}=J_{x}$.

Assume that $P_{1}, P_{2}$ are one-parameter subgroups of $G$ such that for some element $\theta$ in $L, P_{1}^{-}=P_{1} \cup\{\theta\}$ and $P_{2}^{-}=P_{2} \cup\{\theta\}$. Furthermore, assume that $x$ is an element of $L$ such that $\theta x=x \theta \neq x$. Let $\eta_{1}(p)=p x$ for $p \in P_{1}^{-}$and $\eta_{2}(q)=x q$ for $q \in P_{2}^{-}$. Then $\eta_{1}$ and $\eta_{2}$ are homeomorphisms which, as maps into $L$ are closed. In particular, $P_{1}^{-} x$ and $x P_{2}^{-}$are topological closed half-rays in $L$.

As we shall see, $J_{x}$ is necessarily a closed subgroup of $P_{1}$ so that, if it is neither trivial nor all of $P_{1}$, it is an infinite cyclic subgroup. In this case, we can say how $x P_{2}$ is situated relative to $P_{1} x$. To do so, we need to define what we mean by saying one ray spirals around the endpoint of another and of certain arcs going around the endpoint of another.

In the present context, assume that $J_{x}$ is an infinite cyclic subgroup of $P_{1}$ and let $d$ be a generator of $J_{x}$. There is an element $e \in P_{2}$ such that $d x=x e$. Therefore $d^{k} x=x e^{k}$ for each integer $k$. Also $P_{1} x \cap x P_{2}=J_{x} \cdot x$. Let $A$ be the closed interval of $P_{1}$ from 1 to $d$ and let $B$ be the closed interval of $P_{2}$ from 1 to $e$. Thus, for each integer $k$, the set $S_{k}=\left(A d^{k} \cdot x\right) \cup\left(x B \cdot e^{k}\right)$ is a simple closed curve. We will say that the arc $x B e^{k}$ goes around $\theta x$ (relative to $P_{1}^{-} x$ ) provided $\theta x$ lies inside $S_{k}$. We will say that $x P_{2}$ spirals around $\theta x$ (relative to $P_{1} x$ ) provided $x B e^{k}$ goes around $\theta x$ for every integer $k$.

THEOREM 3.7. Let $S$ be a semigroup on a half-space with maximal group G. Let $P_{1}$ and $P_{2}$ be one-parameter subgroups of $G$ such that $P_{1}^{-}=P_{1} \cup\{\theta\}$ and $P_{2}^{-}=P_{2} \cup\{\theta\}$ for some $\theta \in L$. Suppose $x \in L$ is such that $\theta x=x \theta \neq x$. Then $J$ is a closed subgroup of $P_{1}$. If $J$ is a proper subgroup of $P_{1}$ then $x P_{2}$ spirals around $\theta x$ relative to $P_{1} x$.

Proof. Let $\left\{p_{n}\right\}_{n}$ be a sequence in $J$ such that $p_{n} \rightarrow p$ where $p \in P_{1}$. Then there exist elements $q_{n} \in P_{2}$ such that $p_{n} x=x q_{n}$. Thus $x q_{n} \rightarrow p x$. Since the map $\eta_{2}\left(q^{\prime}\right)=x q^{\prime}$, $q^{\prime} \in P_{2}^{-}$, is closed, there exists $q \in P_{2}^{-}$such that $x q_{n} \rightarrow x q$. Hence $p x=x q$. If $q=\theta$ then $p=\theta$ which is false. Thus $q \in P_{2}$ so $p \in J$ and $J$ is a closed subgroup of $P_{1}$.

Suppose $J$ is a proper subgroup of $P_{1}$. Then $J$ is infinite cyclic. Let $d$ be the generator of $J$ which lies between 1 and $\theta$ and order $P_{1}$ so that $d<1$. Let $A$ be the closed interval from $d$ to 1 in $P_{1}$. Let $e$ be the element of $P_{2}$ such that $d x=x e$ and let $B$ be the closed interval of $P_{2}$ between $e$ and 1. Of course $d^{k} x=x e^{k}$ for each integer $k$. Assume that for some integer $k$, the $\operatorname{arc} x e^{k} B$ does not go around $\theta x$. That is, $\theta x$ lies outside the simple closed curve $S_{k}=\left(A d^{k} x\right) \cup\left(x e^{k} B\right)$.

Let $p \in P_{1}$ be between $d$ and 1. Now $p d^{k-1} x P_{2}$ is disjoint from $x P_{2}$ and hence $p d^{k-1} x P_{2}$ is disjoint from $x e^{k} B$. Since $P_{1}\left(p d^{k} x\right) \cap\left(p d^{k} x P_{2}\right)=J_{p d^{k} x} \cdot p d^{k} x=J_{x} \cdot p d^{k} x$ and $P_{1} p d^{k} x=P_{1} x$, we have $P_{1} x \cap\left(p d^{k} x P_{2}\right)=J_{x} \cdot p d^{k} x$ so the nearest points in $P_{1} x \cap p d^{k} x P_{2}$ to $p d^{k} x$ are $p d^{k+1} x$ and $p d^{k-1} x$. Each of these points is a point of $P_{1} x$ lying outside the interval $A d^{k} x$. Hence $p d^{k} x P_{2}$ intersects $S_{k}$ only in $p d^{k} x$. Since $\theta x$ lies outside $S_{k}$ by hypothesis, $d^{k+j} x$ lies outside $S_{k}$ for all $j \geqq 1$. Since 
$p d^{k} x P_{2}$ must get from $p d^{k} x$ to $p d^{k+1} x, p d^{k} x B=p x e^{k} B$ must, except for the endpoint $p d^{k} x$ lie outside $S_{k}$.

Now allow $p$ to approach 1 in $P_{1}$. Clearly the $\operatorname{arcs} p d^{k} x B$ must approach the arc $x e^{k} B$ at least pointwise. But by [10] there is a point of almost uniform convergence which by translation and compactness implies the convergence is in fact uniform. Because of the fact that the $\operatorname{arc} p d^{k} x B$ can not intersect $P_{1} x$ except at $p d^{k} x$ and $p d^{k+1} x$ and lies, except for the point $p d^{k} x$ outside $S_{k}$, this is evidently impossible. We conclude that $\theta x$ is not outside $S_{k}$ for any $k$ so that $x P_{2}$ spirals around $\theta x$ relative to $P_{1} x$.

Now suppose $G \cong \mathrm{Sl}(2)$. Let $P$ be a distinguished one-parameter subgroup of $G$. Let $x \in L \backslash\{0\}$. We know $G x=P x, x G=x P$ and $G x \neq x G$. Let $P_{1}=P_{2}=P$ in the above. Since the center $C$ is contained in $J_{x}, J_{x}$ is nontrivial. Since $G x \neq x G, J_{x}$ is a proper subgroup of $P$. By the preceding theorem, $P x$ must spiral around 0 relative to $x P$. We thus have the following result.

Corollary 3.7.1. Let $S$ be a semigroup on a half-space with $G \cong \mathrm{Sl}$ (2). Let $P$ be a distinguished one-parameter subgroup of $G$. Then for each $x \in L \backslash\{0\}, G x=P x$, $x G=x P$ and $P x$ spirals around 0 relative to $x P$.

THEOREM 3.8. If $G \cong \mathrm{SI}(2)$ then $x y=0$ for all $x, y \in L$.

Proof. We first prove $x^{2}=0$ for all $x \in L$. We know $(G x)^{-}=(P x)^{-}=P^{-} x$ and $(x G)^{-}=(x P)^{-}=x P^{-}$where $P$ is a distinguished one-parameter subgroup of $G$. Now $(G x)^{-}$is a left and $(x G)^{-}$is a right ideal. In particular, $(G x)^{-}$and $(x G)^{-}$ are subsemigroups so $x^{2} \in(G x)^{-} \cap(x G)^{-}$. If $x^{2} \neq 0$ then $x^{2} \in G x \cap x G$. But then $G x \cap x G$ is a group which is impossible. It follows that $x^{2}=0$ for all $x \in L$.

Let $x \in L \mid\{0\}$ and $y \in P x$. Then $y=p x$ for some $p \in P$ so $y x=p x^{2}=0$. Hence the product of any two elements of $P x$ is 0 for any $x \in L$.

Finally, suppose $x, y$ are arbitrary nonzero elements with $P x \neq P y$. We know that $y P$ spirals around 0 relative to $P y$ and hence relative to $P x$. Therefore $P x \cap y P$ $\neq \varnothing$. Hence there exist elements $p_{1}$ and $p_{2} \in P$ such that $p_{1} x=y p_{2}$. Therefore $0=\left(p_{1} x\right)^{2}=\left(p_{1} x\right)\left(y p_{2}\right)=p_{1}(x y) p_{2}$ so $x y=0$.

We summarize all of the results of this section in

THEOREM 3.10. Suppose $S$ is a topological semigroup on a half-space whose maximal group $G$ is isomorphic to $\mathrm{Sl}(2)$. Let $L$ denote the boundary of $G$. Then $L$ contains exactly one idempotent and this is a two-sided zero 0 for $S$. For each $x \in L \backslash\{0\}, \operatorname{dim} G x=\operatorname{dim} x G=1$. Every distinguished one-parameter subgroup $P$ of $G$ has 0 in its closure and if $x \in L \mid\{0\}, G x=P x$ while $x G=x P$. Furthermore, Px spirals around 0 relative to $x P$. If $x, y$ are any two nonzero elements of $L$ then $P x \cap y P \neq \varnothing$. For every $x, y \in L, x y=0$. The cone $\mathscr{C}$ of one parameter subgroups of $G$ which are normal subgroups of planar groups is closed in $S$ and "opens toward" 0.

4. The sets $\xi_{i j}$. If $i, j$ are integers between 0 and 2 , let $\xi_{i j}$ denote the set of idempotents $e$ such that $\operatorname{dim} G e=i$ and $\operatorname{dim} e G=j$, except that $\xi_{11}$ is assumed to include only idempotents $e$ such that $G e=e G$. 
Lemma 4.1. Suppose $\Sigma$ is a topological semigroup containing a commutative planar group $H$ as a dense subgroup. Then there are at most two nonzero idempotents $e \in \Sigma$ such that for some one-parameter subgroup $P \subset H, P^{-}=P \cup\{e\}$.

Proof. Let $e_{1}, e_{2}$ be nonzero idempotents and let $P_{1}, P_{2}$ be one-parameter subgroups of $H$ such that $P_{i}^{-}=P_{i} \cup\left\{e_{i}\right\}$. Note that $e_{1} e_{2}$ is zero for $H$. For $H=P_{1} P_{2}$ so $H e_{1} e_{2}=P_{1} P_{2} e_{1} e_{2}=P_{1} e_{1} P_{2} e_{2}=e_{1} e_{2}$. Now $P_{1}$ and $P_{2}$ divide $H$ into four sets $A_{11}$, $A_{12}, A_{21}$ and $A_{22}$ characterized as follows: every element $x \in H$ has a unique representation of the form $x=p_{1} p_{2}$; with $p_{1} \in P_{1}$ and $p_{2} \in P_{2}$. Place $x \in A_{11}$ iff $p_{1}^{n} \rightarrow e_{1}$ and $p_{2}^{n} \rightarrow e_{2}$. Place $x \in A_{12}$ iff $p_{1}^{n} \rightarrow e_{1}$ and $\left(1 / p_{2}\right)^{n} \rightarrow e_{2} ; x \in A_{21}$ iff $\left(1 / p_{1}\right)^{n} \rightarrow e_{1}$ and $p_{2}^{n} \rightarrow e_{2}$ while $x \in A_{22}$ iff $\left(1 / p_{1}\right)^{n} \rightarrow e_{1}$ and $\left(1 / p_{2}\right)^{n} \rightarrow e_{2}$. Suppose there is a third nonzero idempotent $e_{3}$ such that $e_{3} \in P_{3}^{-}$for some one-parameter group $P_{3}$. We show, however, that $e_{3}$ is not in the closure of any of the sets $A_{i j}$.

Recall from the above that $e_{2} e_{3}=e_{1} e_{3}=0$. If $e_{3} \in\left(A_{22}\right)^{-}$then there exists a sequence $p_{1 n} p_{2 n} \in A_{22}$ such that $p_{1 n} p_{2 n} \rightarrow e_{3}$. Now either $p_{1 n} \rightarrow p_{1} \in P_{1}$ and $1 / p_{2 n} \rightarrow e_{2}$ or, dually, with the roles of $P_{1}$ and $P_{2}$ interchanged, or $1 / p_{1 n} \rightarrow e_{1}$ and $1 / p_{2 n} \rightarrow e_{2}$. In the first case, $\left(1 / p_{1 n}\right)\left(p_{1 n} p_{2 n}\right) .1 / p_{2 n} \rightarrow\left(1 / p_{1}\right) e_{3} e_{2}=0$ while $\left(1 / p_{1 n}\right)$ $\cdot\left(p_{1 n} p_{2 n}\right)=2$. The other two cases yield the same contradiction.

If $p_{1 n} p_{2 n} \in A_{12}$ then either $p_{1 n} \rightarrow p_{1} \in P_{1}$ and $1 / p_{2 n} \rightarrow e_{2}$, or $p_{1 n} \rightarrow e_{1}$ and $p_{2 n} \rightarrow p_{2} \in P_{2}$ or $1 / p_{2 n} \rightarrow e_{2}$ and $p_{1 n} \rightarrow e_{1}$. In the first case, $\left(1 / p_{1 n}\right)\left(p_{1 n} p_{2 n}\right)\left(1 / p_{2 n}\right)$ $\rightarrow\left(1 / p_{1}\right) e_{3} e_{2}=0$. In the second case, $\left(p_{1 n} p_{2 n}\right)\left(1 / p_{2 n}\right) \rightarrow e_{3} p_{2}$ yet $\left(p_{1 n} p_{2 n}\right)\left(1 / p_{2 n}\right) \rightarrow e_{1}$ so $e_{1}=e_{3} p_{2}$ so $e_{1}=e_{1} e_{1}=e_{1} e_{3} p_{2}=0$ which is false. Finally, if $p_{1 n} \rightarrow e_{1}$ and $1 / p_{2 n} \rightarrow e_{2}$ then $\left(p_{1 n} p_{2 n}\right)\left(1 / p_{2 n}\right) \rightarrow e_{3} e_{2}=0$, implying $e_{1}=0$ which is also false.

The other possibilities yield similar contradictions.

THEOREM 4.2. If $H$ is a commutative planar subgroup of $S$ and if $S$ has a zero in $H^{-}$then there is a one-parameter subgroup $P$ of $H$ with $0 \in P^{-}$.

Proof. Let $V$ be a compact neighborhood of 0 as in the proof of Lemma 2.9. Thus, if $x \in V$ then $x^{n} \in V$ for every positive integer $n$ so for every $x \in V$ there is an idempotent $e_{x}$ in the closure of $\left\{x^{n}\right\}_{n}$. If $P_{x}$ is a one-parameter subgroup of $H$ containing $x$ then $P_{x}^{-}=P_{x} \cup\left\{e_{x}\right\}$. By the lemma, there are at most two such idempotents. By choosing $V$ sufficiently small, we may assume that 0 is the only such idempotent in $V$. Thus, if $x \in V$ then $0 \in\left(P_{x}\right)^{-}$.

LEMMA 4.3. Let e be an idempotent in $S$ and suppose $S$ is not one of the semigroups satisfying the conclusions of Theorem 2.5. If $x$ is conjugate to e then $x \in G e \cup e G$. In particular, if $e_{1}$ and $e_{2}$ are idempotents then $e_{1}$ and $e_{2}$ are conjugate if and only if $\left(G e_{1} \cup e_{1} G\right) \cap\left(G e_{2} \cup e_{2} G\right) \neq \varnothing$.

Proof. Under the circumstances, $G e \subset e G$ or $e G \subset G e$ for every idempotent $e \in L$. But then geg $^{-1} \in G e \cup e G$ for any $g \in G$.

THEOREM 4.4. $\xi_{12} \cup \xi_{21}$ contains at most two conjugacy classes of idempotents. 
Proof. Let $e \in \xi_{21}$. Let $H=G_{r}(e)$. Then $e G$ is a group contained in $G e, H^{-}$is a half-plane semigroup isomorphic to Af (1) ${ }^{-}$and $G e$ is filled with conjugates of $e G$, each such conjugate intersecting $H^{-} \cap L$ in exactly one idempotent. Let $P$ be the one-parameter subgroup of $H$ such that $P^{-}=P \cup\{e\}$ and $Q$ be the center of $G$. Then $P Q$ is a commutative nonnormal planar group with $e \in(P Q)^{-}$. We have seen before that the only elements of $(P Q)^{-} \cap G e$ are elements of $e G=e Q$. Hence $(P Q)^{-} \cap(G \cup G e)$ is a semigroup on a half-space.

Next, if $K$ is any commutative nonnormal planar subgroup of $G$ then $K$ is conjugate to $P Q$. Hence there is an idempotent $f \in K^{-} \cap G e$ and a one-parameter subgroup $P_{1}$ of $K$ such that $P_{1}^{-}=P_{1} \cup\{f\}$.

The remarks proved so far have obvious left-right duals if $e \in \xi_{21}$ instead of $\xi_{12}$. Suppose there exist as many as three elements $e_{1}, e_{2}, e_{3} \in \xi_{12} \cup \xi_{21}$, no two of which are conjugate. By the previous lemma, if $i \neq j$ then $\left(G e_{i} \cup e_{i} G\right) \cap\left(G e_{j} \cup e_{j} G\right)$ $=\varnothing$. Let $K$ be a commutative, nonnormal planar subgroup of $G$. Since any two such groups are conjugate, the previous arguments show that for each $i=1,2,3$, $K$ contains a one-parameter group running to some idempotent in $G e_{i} \cup e_{i} G$. This means that $K$ contains three one-parameter subgroups running to nonzero idempotents in the boundary, contrary to the first lemma of this section.

Corollary 4.4.1. If $G$ is not commutative then $\left|\xi_{12} \cup \xi_{21} \cup \xi_{22}\right| \leqq 2$ where, in general, $|A|$ denotes the number of conjugacy classes of elements of $A$.

Proof. If $\xi_{12} \cup \xi_{21}=\varnothing$ the result is immediate since if $\left|\xi_{22}\right| \geqq 3$ then $G$ is commutative. If $\xi_{12} \cup \xi_{21} \neq \varnothing$ then $G$ is isomorphic to $R \times \operatorname{Af}(1)$. We know in any case that $\left|\xi_{12} \cup \xi_{21}\right| \leqq 2$. If $\left|\xi_{12} \cup \xi_{21}\right|=2$ then $\xi_{22}=\varnothing$. For whenever $\xi_{12} \cup \xi_{21}$ $\neq \varnothing$, the one-parameter subgroup of $G$ which forms the normal one-parameter group of every copy of Af (1) in $G$ is closed in $S$ (it is closed in $H^{-}$, for example, if $\left.H=G_{l}(e), e \in \xi_{12}\right)$. Thus, if $e \in \xi_{22}$ then $G_{l}(e)$ is the center $Q$ of $G$ since the center is then the only other normal one-parameter subgroup of $G$. This shows, incidentally, that whenever $\xi_{12} \cup \xi_{21} \neq \varnothing$ then $\left|\xi_{22}\right| \leqq 1$. Assume, again, that $\left|\xi_{12} \cup \xi_{21}\right|=2$, but that $\xi_{22} \neq \varnothing$ with $e \in \xi_{22}$. Since $Q$ belongs to every commutative planar $K$, $e \in K^{-}$for every such $K$. On the other hand, if $e_{2} \in \xi_{12} \cup \xi_{21}$ then $K^{-} \cap\left(G e_{2} \cup e_{2} G\right)$ $\neq \varnothing$. Hence, if $\left|\xi_{12} \cup \xi_{21}\right|=2$ then $K^{-}$contains three one-parameter subgroups running to idempotents in the boundary in violation of Lemma 4.1.

If $\left|\xi_{12} \cup \xi_{21}\right|=1$, the conclusion is immediate since then $\left|\xi_{22}\right| \leqq 1$.

Consider the collection $\xi_{11}$. If for any $e \in \xi_{11}$, Ge is closed then $\xi_{11}=\{e\}$. For suppose $e_{2}$ is a second such idempotent. Since $G e=e G$ and $G e$ is closed, $G e$ is a two-sided ideal. Therefore $G e \cap\left(G e_{2}\right)^{-} \neq \varnothing$ so $G e \subset\left(G e_{2}\right)^{-}$. But for any $e_{2} \in \xi_{11}$, $\left(G e_{2}\right)^{-}$consists of $G e_{2}$ and at most one additional point which is a zero for $S$.

Thus assume $G e$ is not closed for some (and hence all) $e \in \xi_{11}$. Then $S$ has a two-sided zero 0 and $(\mathrm{Ge})^{-}=G e \cup\{0\}$ for each $e \in \xi_{11}$. It follows that if $e_{1}$ and $e_{2}$ are any two elements of $\xi_{11}$ then $e_{1} e_{2}=e_{2} e_{1}=0$. For $e_{1} e_{2} \in\left(G e_{1}\right)^{-} \cap\left(G e_{2}\right)^{-}=\{0\}$ and similarly for $e_{2} e_{1}$. 
If $e \in \xi_{11}$ let $H_{e}$ denote the isotropy group of $G$ at $e$ and let $T_{e}=H_{e}^{-} \cap L=H_{e}^{-} \backslash H_{e}$. Alternately, if in some notation, some member of $\xi_{11}$ is denoted $e_{i}$, set $H_{e_{i}}=H_{i}$ and $T_{e_{i}}=T_{i}$. If $e_{1}$ and $e_{2}$ are distinct members of $\xi_{11}$ then $H_{1} \neq H_{2}$ since if $H_{1}=H_{2}$ then $H_{1}$ has two two-sided zeros $e_{1}$ and $e_{2}$. Neither can $e_{1}$ belong to $T_{2}$. For suppose $e_{1} \in T_{2}$. Since $e_{2}$ is a two-sided zero for $H_{2}^{-}, e_{2} e_{1}=e_{2}$ while as just shown, $e_{2} e_{1}=0$. In fact, $G e_{1} \cap T_{2}=\varnothing$. For if $g e_{1} \in T_{2}$, then $g e_{1} e_{2}=e_{2}$, though $e_{1} e_{2}=0$.

Let $e_{1}, e_{2} \in \xi_{11}$ and assume $T_{1} \cap T_{2} \neq \varnothing$. Let $x \in T_{1} \cap T_{2}$ and let $A_{i}$ denote the component of $T_{i} \mid\left\{e_{i}\right\}$ containing $x$. According to the known structures of semigroups on a half-plane, either $A_{i}$ is a group or $A_{i}^{2}=\left\{e_{i}\right\}$ and if $A_{i}$ is a group then $H_{i}$ is commutative. Suppose $A_{i}^{2}=\left\{e_{i}\right\}$. Then $e_{i}=x^{2} \in T_{1} \cap T_{2}$ which is contrary to what was just proved. Thus $A_{1}$ and $A_{2}$ are both groups and $H_{1}$ and $H_{2}$ are normal (since $G e_{i}=e_{i} G$ ) commutative planar groups. The only groups on $E_{3}$ which contain as many as two normal commutative planar groups are the two nilpotent groups so if $T_{1} \cap T_{2} \neq \varnothing, G$ is nilpotent.

Let $f_{i}$ denote the identity of $A_{i}$ with $A_{i}$ and $x$ as above. Since $f_{i} \in H_{i} x, H_{i} f_{i}=H_{i} x$ for $i=1,2$, so $G x \supset H_{1} f_{1} \cup H_{2} f_{2}$. Therefore $\operatorname{dim} G x=2$. Similarly $\operatorname{dim} x G=2$. The only time a two-dimensional left orbit of $G$ can contain as many as two idempotents is when the corresponding right orbit is one or zero dimensional. Since $\operatorname{dim} G x=\operatorname{dim} x G=2$ and the idempotents $f_{1}$ and $f_{2}$ belong to $G x, f_{1}=f_{2}$. Thus $G x=G f_{1}=f_{1} G$ and $G f_{1}$ is a planar group which, being homomorphic to a nilpotent group is abelian. Furthermore, $e_{1}, e_{2}$ and 0 lie in the boundary of $G f_{1}$. Thus $G e_{1} \cup G e_{2} \cup\{0\}$ forms the complete boundary of $G f_{1}$. It follows that $T_{1} \cap G f_{1}$ and $T_{2} \cap G f_{1}$ are one-parameter subgroups of $G f_{1}$ so $\left(T_{1} \cap G f_{1}\right) \cap\left(T_{2} \cap G f_{2}\right)=\left\{f_{1}\right\}$. Therefore $T_{1} \cap T_{2} \cap G f_{1}=\left\{f_{1}\right\}$ and $x=f_{1}$.

What we have proved so far shows that $T_{1} \cap T_{2}$ consists of at most two idempotents. Suppose there are in fact two idempotents $f_{1}$ and $g_{1}$ in $T_{1} \cap T_{2}$. Then $H_{1}^{-}$ is a semigroup on a half-plane with zero $e_{1}$ so $f_{1} g_{1}=e_{1}$. But $H_{2}^{-}$is also a semigroup on a half-plane with zero $e_{2}$ so $f_{1} g_{1}=e_{2}$ which is a contradiction.

We pause to summarize what has been proved up to this point:

THEOREM 4.5. Let $\xi_{11}$ denote the set of idempotents $e$ such that $\operatorname{dim} G e=1$ and $G e=e G$. If for any $e \in \xi_{11}, G e$ is closed, $\xi_{11}=\{e\}$. If $\xi_{11}$ contains more than one element then $S$ has a two-sided zero 0 and for each $e \in \xi_{11},(G e)^{-}=G e \cup\{0\}$. If $e_{1}, e_{2}$ are distinct members of $\xi_{11}$ then $e_{1} e_{2}=0$. For such $e_{1}, e_{2}$, set $H_{i}=G_{l}\left(e_{i}\right)$ and $T_{i}=\left(H_{i}\right)^{-} \cap L$. Then $H_{1} \neq H_{2}$ and $G e_{1} \cap T_{2}=\varnothing$. If $T_{1} \cap T_{2} \neq \varnothing$ then $T_{1} \cap T_{2}$ consists of a single idempotent $f$ and $(G f)^{-}=G f \cup G e_{1} \cup G e_{2} \cup\{0\}$. Moreover, in this case, $H_{1}$ and $H_{2}$ are abelian and $G$ is nilpotent.

If $\xi_{11}$ contains three distinct elements $e_{1}, e_{2}, e_{3}$ such that for each $i, j=1,2,3$, $T_{i} \cap T_{j} \neq \varnothing$ then evidently $L=G f_{12} \cup G f_{23} \cup G f_{13} \cup G e_{1} \cup G e_{2} \cup G e_{3} \cup\{0\}$ where $f_{i j}$ is the idempotent in $T_{i j}$ for $i, j=1,2,3, i \neq j$. That is, $L$ is isomorphic to the boundary of the maximal group in $P^{-} \times P^{-} \times P^{-}$where, as usual, $P^{-}$denotes a copy of the nonnegative reals under multiplication. Presumably $S$ itself is isomorphic 
to $P^{-} \times P^{-} \times P^{-}$. Certainly, $G$ is abelian. For $G$ is already nilpotent and $\left\{G_{l}\left(f_{i j}\right)\right\}_{i, j}$ is a set of three normal one-parameter subgroups of $G$. But we do not go further into this matter now. In any event, $\xi_{11}=\left\{e_{1}, e_{2}, e_{3}\right\}$ and we state these results as a theorem:

THEOREM 4.6. Suppose $\xi_{11}$ contains distinct idempotents $e_{1}, e_{2}, e_{3}$ such that for each $i, j=1,2,3, T_{i} \cap T_{j} \neq \varnothing$. Let $f_{i j}$ denote the idempotent in $T_{i} \cap T_{j}$ for $i \neq j$. Then $G$ is abelian and $L$ is isomorphic to the boundary of the maximal group in $P^{-} \times P^{-} \times P^{-}$. In particular, $L=\left(\bigcup_{i, j} G f_{i j}\right) \cup\left(\bigcup_{i} G e_{i}\right) \cup\{0\}$.

THEOREM 4.7. Except in the case where there exists an idempotent e with $\operatorname{dim} G e$ $=\operatorname{dim} e G=1$ but $G e \neq e G$, the number of conjugacy classes of idempotents in $L$ is countably infinite. If there are no nilpotent elements, this number is finite.

Proof. Of course $\left|\xi_{12} \cup \xi_{21}\right| \leqq 2$ and $\left|\xi_{22}\right|$ is countable since $\left\{G e: e \in \xi_{22}\right\}$ is a collection of open, pairwise disjoint subsets of $L$. It remains to prove $\left|\xi_{11}\right|$ is countable. We have seen that if $e \in \xi_{11}$ and $T$ is the boundary of $G_{l}(e)$ then $G T$ is a neighborhood of $e$ and hence of $G e$ (Corollary 2.3.1). Furthermore, $G T$ can contain at most three idempotents- $e$ and possibly one in each component of $T \backslash\{e\}$. Each of the latter, if any, belongs to $\xi_{22}$. Therefore $G T$ is a neighborhood of $G e$ which is disjoint from $G f$ for every other $f \in \xi_{11}$. If $\xi_{11}$ were uncountable, the sets $\left\{G e: e \in \xi_{11}\right\}$ would cluster at some $G e_{0}, e_{0} \in \xi_{11}$ which we have just seen is impossible.

If the number of conjugacy classes is infinite then $\left|\xi_{11} \cup \xi_{22}\right|$ is infinite. Whether $\left|\xi_{11}\right|$ or $\left|\xi_{22}\right|$ is infinite we can select a sequence of rays $\left\{\left(G x_{i}\right)^{-}\right\}_{i}$ emanating from 0 such that $g x_{i} \cdot g x_{j}=0$ for each $i \neq j$. This sequence will cluster at some $y \neq 0$ and $y^{2}=0$.

Remark. We do not explore the extent to which $\left|\xi_{11} \cup \xi_{22}\right|$ can be infinite. In $\S 7$ we show that $\left|\xi_{11} \cup \xi_{22}\right|$ can be arbitrarily large even in the abelian, nonnilpotent case.

Corollary 4.7.1. If $S$ contains a two-sided zero 0 then there exists a oneparameter subgroup $P$ of $G$ such that $0 \in P^{-}$. Hence if $e$ is any idempotent in $L$ there exists a one-parameter subgroup $P$ of $G$ such that $P^{-}=P \cup\{e\}$.

Proof. If $G$ is isomorphic to $\mathrm{Sl}(2)$, we already have the desired conclusion. Assume that $G$ is not isomorphic to $\mathrm{Sl}$ (2). We shall use the fact that then every element of $G$ belongs to a one-parameter group, a fact used before which can be proved directly in each case, or derived from the fact that every such $G$ is some kind of semidirect product of the one dimensional vector group and a planar group.

Consider two cases according to whether $\xi_{12} \cup \xi_{21}$ is empty or not. First suppose $\xi_{12} \cup \xi_{21} \neq \varnothing$. To be definite, take $e \in \xi_{21}$. Then $G \cong R \times \operatorname{Af}(1)$ and $G_{r}(e)$ is a noncommutative planar group. Let $P_{1}$ be the one-parameter subgroup of $G_{r}(e)$ such that $e \in P_{1}^{-}$. Let $Q$ denote the center of $G$. Then $0 \in(e G)^{-}=(e Q)^{-} \subset\left(P_{1} Q\right)^{-}$and $P_{1} Q$ is a commutative planar group. By Theorem 4.2 above, $P_{1} Q$ and hence $G$, contains a one-parameter subgroup $P$ with $0 \in P^{-}$. 
If $\xi_{12} \cup \xi_{21}=\varnothing$ then every nonzero idempotent belongs to $\xi_{11} \cup \xi_{22}$. Moreover, $\xi_{11} \cup \xi_{22}$ is countable. Choose a neighborhood $V$ of 0 having the property that if $x \in V \cap G$ then $x^{n} \in V$ for every positive integer $n$. It follows that if $x \in V$ and $P_{x}$ is a one-parameter subgroup of $G$ containing $x$ then $P_{\bar{x}} \cap L$ contains an idempotent. Since $V$ can not be covered by a countable number of one-parameter groups, there exists an $x \in V$ such that no idempotent in $\xi_{11} \cup \xi_{22}$ is in $P_{x}^{-}$. Thus $0 \in P_{x}^{-}$. The rest of the conclusion is immediate from what has gone before.

5. The position of orbits. This is a short section in which it is proved for orbits generally what has already been proved for orbits through idempotents: to wit, that they are all nicely embedded.

THEOREM 5.1. Suppose $\operatorname{dim} G x=2$. Suppose $G x$ is not closed and let $\partial(G x)=$ $(G x)^{-} \mid G x$. Then $\partial G x$ is either a point, a line or a half-ray. If $e$ is an idempotent in $\partial G x$ such that $G e$ is closed then either $G e=\partial G x$ (and $e G=e$ or $G e=e G$ ) or $G e=e$ and $e G$ is a line or e is a two-sided zero for $S$. If $\partial G x$ consists of a single point, $S$ has a two-sided zero 0 and $\partial G x=\{0\}$. Furthermore, in this case, $L^{2}=\{0\}$.

Proof. We know there exists an idempotent $e$ in $\partial G x$ such that $G e$ is closed. Assume $\operatorname{dim} G e=1$. If $\operatorname{dim} e G=2$ then $e G=L$ and every left orbit is one-dimensional. Therefore, $\operatorname{dim} e G \leqq 1$. If $\operatorname{dim} e G=0, G e$ is the entire boundary of $G x$ by Theorem 2.11. If $\operatorname{dim} e G=1$ then $G e=e G$. Thus $G e$ is a two-sided ideal in $S$ and $H=G_{l}(e)$ is a normal planar group such that $H^{-}$is a half-plane with $e$ for two-sided zero and $H^{-}$forms a local cross-section at $e$ for the left orbits of $P$ where $P$ is a one-parameter group in $G$ with $G=P H$. Suppose there exists $y \in \partial(G x) \backslash G e$. Since any left ideal meets any right ideal, $G e \cap(G y)^{-} \neq \varnothing$ so $e \in(G y)^{-}$. Since $H^{-}$forms a local cross-section at $e$ to the orbits of $P$, we may assume $y \in H^{-}$. Therefore, since $H^{-}$is a half-plane, $(H y)^{-}=H y \cup\{e\}$ so $H y=G y$ and $(G y)^{-}=H y \cup\{e\}$ which is contrary to the fact that $G e \subset(G y)^{-}$. It follows in this case that $\partial(G x)=G e$.

Suppose $\operatorname{dim} G e=0$. Thus $e G$ is closed so $\operatorname{dim} e G \neq 2$. If $\operatorname{dim} e G=1$ then $L \backslash e G$ is the union of two right orbits and $e G$ forms their common boundary. Thus $G x=x G$ since $\operatorname{dim} G x=2$, so $G x$ has $e G$ for its boundary.

Suppose $\partial(G x)$ contains a two-sided zero 0 . Choose a one-parameter group $P$ in $G$ with $P^{-}=P \cup\{0\}$. For any $y \in \partial(G x), y \neq 0, G y=P y$ so $(G y)^{-}=(P y)^{-}=P^{-} y$. It is thus clear in this case that $\partial(G x)$ consists either of 0 alone, a half-ray or the union of two half-rays.

Suppose $\partial G x$ consists of a single point. This point must obviously be an idempotent $e$ such that $G e=e$. Given the alternatives mentioned above, it follows that $e G=e$ so $e$ is a two-sided zero 0 for $S$.

In this case, $x^{2}=0$. For $x G \subset G x$. If $x^{2} \in x G$ then $x G$ and hence $G x$ is generated by an idempotent which is impossible. Therefore $x^{2}=0$. Let $u, v$ be nonzero elements of $L$. Then there exist elements $g_{1}, g_{2}, g_{3} \in G$ such that $u=g_{1} x, v=g_{2} x$ and $x g_{2}=g_{3} x$. Hence $u v=g_{1} g_{3} x^{2}=0$ so $L^{2}=\{0\}$. 
At present, we do not know whether there is a semigroup on a half-space with $G x=L \backslash\{0\}$ for some $x$. If there is such an example with $G$ commutative then there is in addition the following pathological situation: There is a planar group $H$ in $G$ such that $H^{-} \supset L$. For let $P$ be a one-parameter subgroup of $G$ with $0 \in P^{-}$. Choose a second one-parameter group $Q$ such that the component of the identity of $G_{l}(x)$ is not contained in $P Q$. Let $H=P Q$. Then $0 \in H^{-}$. Since $H^{-}$cannot consist of just one additional point, there is a nonzero element $y \in H^{-} \cap L$. But $y \in G x$ $=H \cdot G_{l}(x) x=H x$ so $y \in H x$ and $x \in H^{-}$. Therefore $G x \subset H^{-}$and $L \subset H^{-}$.

THEOREM 5.2. Let $x \in L$ and assume $\operatorname{dim} G x=1$. If $G x$ is not closed, then there exists an idempotent e such that $(G x)^{-}=G x \cup\{e\},(G x)^{-}$is a half-ray, $G e=e$ and $\operatorname{dim} e G \leqq 1$. If $\operatorname{dim} x G=1$ also then $S$ has a two-sided zero 0 and $(G x)^{-}=G x \cup\{0\}$ while $(x G)^{-}=x G \cup\{0\}$. In any case, if $S$ has a two-sided zero 0 and $\operatorname{dim} G x=1$ then $(G x)^{-}=G x \cup\{0\}$.

Proof. There exists an idempotent $e \in(G x)^{-} \mid G x$ such that $G e$, and hence $e G$, is closed. Certainly $\operatorname{dim} G e \neq 2$. Suppose $\operatorname{dim} G e=1$. If $\operatorname{dim} e G=2$ then $e G=L$ so every left orbit of $G$, being a right multiple of a member of $G$ is closed. Thus, $\operatorname{dim} e G \leqq 1$. If $\operatorname{dim} e G=1$ then $G e=e G$ since otherwise all left and right orbits of $G$ are closed by Theorem 2.5. Therefore $G e=e G$ so $G e$ is a group and if $H=G_{l}(e)$ then $H$ is a normal planar subgroup of $G$ such that $e \in H^{-}$and $H^{-}$is a half-plane. Furthermore, $H^{-}$provides a local cross-section at $e$ to the orbits of $P$ where $P$ is a one-parameter subgroup with $G=P H=H P$. Since $G e \subset(G x)^{-}$and $G e$ has points on both sides of $H^{-} \cap L, G x$ intersects $H^{-} \cap L$ so we may suppose $x \in H^{-} \cap L$. However, since $H^{-}$is a semigroup on a half-plane with two-sided zero $e,(H x)^{-}=$ $H x \cup\{e\}$. Since $G x$ is a line containing $H x$ and $G x \cap G e=\varnothing,(G x)^{-}=(H x)^{-}$, whence $(G x)^{-}=G x \cup\{e\}$ which is a contradiction. Therefore $\operatorname{dim} e G \neq 1$ so $\operatorname{dim} e G=0$. But if $\operatorname{dim} G e=1$ and $\operatorname{dim} e G=0$ then $L \backslash G e$ is the union of two twodimensional right orbits which is a contradiction since $\operatorname{dim} G x=1$.

We conclude that $\operatorname{dim} G e=0$. Furthermore, $\operatorname{dim} e G \neq 2$. Since, again, in that case all left and right orbits of $G$ are closed. Therefore $\operatorname{dim} e G \leqq 1$.

Of course, if $\operatorname{dim} e G=0$ then $e$ is a two-sided zero and if $S$ has a zero then $e=0$ since there cannot be distinct left and right zeros in a semigroup.

We must show $(G x)^{-}=G x \cup\{e\}$. Suppose first that $\operatorname{dim} e G=1$. By Theorem $2.11, L \mid e G$ is the union of two right orbits of $G$. Now $x \notin e G$ since then $g x=x$ for all $g \in G$. Thus $x G$ must coincide with the component of $L \backslash e G$ containing $x$. Since $\operatorname{dim} G x=1$, every left orbit of $G$ in $x G$ is one-dimensional. Furthermore, the left orbits of $G$ in $x G$ are just the portions of the various conjugates of $\left(G_{r}(e)^{-}\right) \cap L$ which lie in $x G$. The closures of each of these are half-rays emanating from points of $e G$. Since $e \in(G x)^{-},(G x)^{-}=G x \cup\{e\}$ in this case.

Suppose $\operatorname{dim} e G=0$ so $e$ is a two-sided zero. Let $P$ be a one-parameter subgroup of $G$ with $0 \in P^{-}$. Then $P$ has no conjugates in $G_{l}(x)$ since if so, $0 \in G_{l}(x)^{-}$which is false. Therefore $G x=P x$ and $(G x)^{-}=(P x)^{-}=P x \cup\{e\}, \operatorname{so}(G x)^{-}=G x \cup\{e\}$ in this case also. 
Of course, if $\operatorname{dim} G x=\operatorname{dim} x G=1$ and $G x$ is not closed then $S$ has a two-sided zero 0 and $(G x)^{-}=G x \cup\{0\}$ while $(x G)^{-}=x G \cup\{0\}$. For by what has just been proved, there exists a left zero $e$ such that $(G x)^{-}=G x \cup\{e\}$. By the dual to this result, there exists a right zero $f$ such that $(x G)^{-}=x G \cup\{f\}$. If a semigroup has a left zero and a right zero, the two are equal so $e=f$ and $e$ is a two-sided zero.

6. The radical. An element $x \in S$ is nilpotent if $S$ has a two-sided zero 0 and $x^{n}=0$ for some positive integer $n$. An element $x$ of $S$ is weakly left nilpotent if $\operatorname{dim} G x^{n}<\operatorname{dim} G x$ for some positive integer $n$. If $\operatorname{dim} x^{n} G<\operatorname{dim} x G$ for some $n, x$ is weakly right nilpotent.

Recall that for each $x \in L,(G x)^{-} \mid G x$ is a closed left ideal and $(x G)^{-} \mid x G$ is a closed right ideal. For example, that $(G x)^{-} \mid G x$ is closed is due to the fact that $G x$ is locally compact and that a locally compact space is open in its closure. The rest is clear since $G\left((G x)^{-} \mid G x\right) \subset(G x)^{-} \backslash G x$.

THeOREM 6.1. Let $x \in S$. Then $x^{2} \in G x$ if and only if $x^{2} \in x G$. Thus, if $x^{n} \in G x \cup x G$ for some positive integer $n$ then $x^{2} \in G x \cap x G, G x \cap x G$ is a group and the left as well as the right $G$-orbit through $x$ contains an idempotent.

Proof. The first assertion is true if $G x$ is closed since then $x G$ is also closed and we always have $x^{2} \in(G x)^{-} \cap(x G)^{-}$. A similar statement holds if $x G$ is closed. Assume that neither $x G$ nor $G x$ is closed. Therefore $\operatorname{dim} G x \neq 0 \neq \operatorname{dim} x G$. If $\operatorname{dim} G x=\operatorname{dim} x G=2$ then $G x=x G$ and the first assertion is clear in this case. Suppose $\operatorname{dim} G x=1$ and $\operatorname{dim} x G=2$. Then $G x \subset x G$. If $x^{2} \in G x$ then certainly $x^{2} \in x G$. Suppose $x^{2} \in x G$. Since $\operatorname{dim} G x=1$ but $G x$ is not closed, $(G x)^{-}=G x \cup\{e\}$ for some idempotent $e$ where $G e=e$. Thus, if $x^{2} \notin G x$ then $x^{2}=e$. But then $e \in x G$ so $x G$ and hence $G x$ is closed which is a contradiction. A similar argument applies if $\operatorname{dim} G x=2$ and $\operatorname{dim} x G=1$. Finally, suppose $\operatorname{dim} G x=\operatorname{dim} x G=1$. Then $S$ has a two-sided zero 0 and $(G x)^{-}=G x \cup\{0\}$ while $(x G)^{-}=x G \cup\{0\}$. From this, it is clear that $x^{2} \in G x$ if and only if $x^{2} \in x G$ in this case, so the proof of the first assertion is complete.

Since $(G x)^{-} \mid G x$ is a left ideal and $(x G)^{-} \backslash x G$ is a right ideal, if $x^{2} \notin G x \cup x G$ then $x^{n} \notin G x \cup x G$ for any positive integer $n$ so we have the first part of the second assertion.

We have already seen that if $x^{2} \in G x \cap x G$ then $G x \cap x G$ contains an idempotent and is a group.

Note that $\operatorname{dim} G x^{n}<\operatorname{dim} G x$ if and only if $x^{n} \notin G x$ since all orbits of $G$ are regularly embedded. Thus, the previous result yields the

COROLlaRY 6.1.1. Weak left and right nilpotency are equivalent notions in L.

Hereafter we shall refer to a weakly left or right nilpotent element as a weakly nilpotent element. The notion of weak nilpotency is in some respects more critical than nilpotency but it is useful to study the latter notion first.

Lemma 6.2. If $e$ is an idempotent then Ge is a semigroup. 
Proof. If $\operatorname{dim} G e=\operatorname{dim} e G=2$ then $G e=e G$ and $G e$ is a group. If $\operatorname{dim} G e=1$ and $\operatorname{dim} e G=2$ then $G e \subset e G$ and $G e$ is a group. Suppose $\operatorname{dim} G e=2$ and $\operatorname{dim} e G=1$. Let $H=G_{r}(e)$. We know $G \cong R \times \operatorname{Af}(1), H$ is a noncommutative planar group, $H^{-}$is isomorphic to $\mathrm{Af}(1)^{-}$and every element of $G e$ can be represented in the form $h p$ where $h \in H^{-} \cap L$ and $p$ belongs to the center of $G$. Since multiplication in $H^{-} \cap L$ satisfies " $x y=x$ ", if $h_{1} p_{1}$ and $h_{2} p_{2}$ are arbitrary elements of $G e$ then $h_{1} p_{1} h_{2} p_{2}=h_{1} h_{2} p_{1} p_{2}=h_{1} p_{1} p_{2} \in G e$ so $G e$ is a semigroup.

If $\operatorname{dim} G e=\operatorname{dim} e G=1$ and $G e=e G$ then $G e$ is a group. If $G e \neq e G$ then $\{x y\}=$ $x G \cap G y$ for all $x, y \in L$. In particular if $g_{1}, g_{2} \in G$ then $\left\{g_{1} e \cdot g_{2} e\right\}=g_{1} e G \cap G g_{2} e$ $=g_{1} e G \cap G e \subset G e$ so $G e$ is a semigroup.

Other cases are either immediate, or analagous to those already considered.

THEOREM 6.3. If $S$ has a zero 0 and $x^{2}=0$ then $a b=0$ for all $a, b \in(S x \cup x S)^{-}$.

Proof. We prove first that if $x^{2}=0$ then $a^{2}=0$ for all $a \in G x \cup x G$. Suppose $\operatorname{dim} G x=1$ and let $g \in G$. If $(g x)^{2} \neq 0$ then $(g x)^{2} \in G x$ so $G \cdot g x=G x$ contains an idempotent $e$ and $G x=G e$. But $G e$ is a semigroup so $x^{2} \in G e$ which is a contradiction.

A similar argument shows that if $\operatorname{dim} x G=1$ then if $g \in G,(x g)^{2}=0$ so $x g x=$ $(x g x g) g^{-1}=0$ whence $(g x)^{2}=0$. Based on a dual argument which we do not give, we conclude that if $\operatorname{dim} G x=1$ or $\operatorname{dim} x G=1$ then $a^{2}=0$ for all $a \in G x \cup x G$.

Since there is a two-sided zero, the only other case to be considered is that in which $\operatorname{dim} G x=\operatorname{dim} x G=2$. In this case, $G x=x G$ so clearly $a^{2}=0$ for any

$$
a \in G x \cup x G .
$$

Note next that if $x^{2}=0$ then $x g x=0$ for all $g \in G$ since $g x g x=0$ so $x g x=g^{-1}(g x g x)$ $=0$. It is now easy to see that $a b=0$ for all $a, b \in G x \cup x G$. Thus $a b=0$ if $a, b \in(G x)^{-} \cup(x G)^{-}$so $a b=0$ if $a, b \in S x \cup x S$ and hence the final conclusion.

If $x^{n}=0$ for some positive integer $n$ then $x^{4}=0$. Indeed, if $x^{n}=0$ but $x^{2} \neq 0$ then $\operatorname{dim} G x=\operatorname{dim} x G=2$ since, for example, if $\operatorname{dim} G x=1$ and $x^{2} \neq 0$ then $x^{2} \in G x$ so $G x=G e$ for some idempotent and $G e$ is a semigroup. Furthermore, if $\operatorname{dim} G x=$ $\operatorname{dim} x G=2$ and $x^{2} \neq 0$ then $x^{2} \in(G x)^{-} \mid G x$. We know that when $\operatorname{dim} G x=2$, the boundary $\partial G x$ of $G x$ consists either of 0 alone, or 0 plus a set $A$ where $A=G a=a G$ for $a \in A$ or 0 plus two sets $A, B$ where $A=G z=a G$ for $a \in A$ while $B=G b=b G$ for $b \in B$. When $x^{2} \neq 0$, we may suppose $x^{2} \in A$. But $\operatorname{dim} G x^{2}=1$ so if $\left(x^{2}\right)^{2} \neq 0$ then $A$ is a group and $\left(x^{2}\right)^{m} \neq 0$ for any positive integer $m$ which is false when $x^{n}=0$. We can say somewhat more:

THEOREM 6.4. If $x^{n}=0$ for some positive integer $n$ then $x^{3}=0$. If $x^{2} \neq 0$ then $\operatorname{dim} G x=\operatorname{dim} x G=2$. Let $\partial G x=A \cup B \cup\{0\}$ as described above except that $B$ may be empty. Then $(G x)^{2} \subset A$ and $(G x) A=A(G x)=\{0\}$. For any $a, b, c \in(G x)^{-}, a b c=0$.

Proof. We already know that if $x^{n}=0$ then $x^{4}=0$ and if $x^{2} \neq 0$ then $\operatorname{dim} G x$ $=\operatorname{dim} x G=2$ and the boundary $\partial G x$ of $G x$ consists of 0 together with a set $A$, or 0 and two sets $A, B$ where both $A$ and $B$ are left as well as right one-dimensional 
$G$-orbits. Suppose $x^{2} \in A$. Since $A^{-}$is a two-sided ideal, if $x^{3} \neq 0$ then $x^{3}=g x^{2}$ for some $g \in G$. But then $0=x^{4}=g x^{3}$ so $x^{3}=0$. In any case, $x^{3}=0$.

If $a, b \in G x$ then we may write $a=g x$ and $b=x h$ for some $g, h \in G$ so $a b=g x^{2} h \in A$ since $A$ is both a left and right $G$-orbit. If $a \in A, b \in G x$, write $a=g x^{2}$ and $b=x h$ so $a b=g x^{2} x h=0$. A similar argument shows $b a=0$. It follows that $a b c=0$ for all $a, b, c \in(G x)^{-}$.

Call a left, right or two-sided ideal I nilpotent if there exists a positive integer $n$ such that $x_{1} x_{2} \ldots x_{n}=0$ for any set $x_{1}, \ldots, x_{n}$ of $n$ elements of $I$.

THEOREM 6.5. The set $N$ of nilpotent elements of $S$ is a closed two-sided ideal which contains every nilpotent left ideal as well as every nilpotent right ideal. Moreover, the product of any three elements of $N$ is zero.

Proof. Let $x \in N$. If $x^{2}=0$, we have seen that $(S x \cup x S)^{2}=0$. Suppose $x^{3}=0$ but $x^{2} \neq 0$. Then $\operatorname{dim} G x=\operatorname{dim} x G=2, S x \cup x S \subset(G x)^{-}$and the product of any three elements of $(G x)^{-}$is zero. Thus, in any case, if $x \in N$ then $S x \cup x S \subset N$ so $N$ is a two-sided ideal. Since $x^{3}=0$ for all $x \in N, N$ is obviously closed.

We prove that the product of any three elements of $N$ is 0 in a series of steps which we number for easy reference.

1. If $x^{2}=0=y^{2}$ then either $x y=0$ or $\operatorname{dim} x G=\operatorname{dim} G y=2$,

$$
x y \in\left((x G)^{-} \cap(G y)^{-} \backslash(x G \cup G y)\right),
$$

$G x y=x y G$ and $x y x=0=y x y$.

Proof. Always $x y \in(x G)^{-} \cap(G y)^{-}$. If $x y=x g$ then $x=x y g^{-1}$ so $x y=x y g^{-1} y$. But $y g^{-1} y=0$ since $y^{2}=0$. A similar argument applies if $x y \in G y$.

If $x y \neq 0$ and $x y \notin x G \cup G y$ then $\operatorname{dim} x G=\operatorname{dim} G y=2$. If $x G=G y$ then $x y=0$ so if $x y \neq 0$ then $x G \cap G y=\varnothing$ since when $\operatorname{dim} x G=\operatorname{dim} G y=2$ and $x G \cap G y \neq \varnothing$, $x G=G y$. Thus if $x y \neq 0$ then $x y$ is a common nonzero boundary point of $x G$ and $G y$. In general, if $\operatorname{dim} G u=2$ and $v$ is a nonzero boundary point of $G u$ then $\operatorname{dim} v G \leqq 1$ so $v G \subset G v$. Similarly, if $\operatorname{dim} u G=2$ and $v$ is a nonzero boundary point then $G v \subset v G$. Thus $G x y=x y G$ and $\operatorname{dim} G x y=1$. Finally, $x y x=y x y=0$ since, for example $x y \in(x G)^{-}$and $\left((x G)^{-}\right)^{2}=\{0\}$.

2. If $u^{2}=0, G u=u G, \operatorname{dim} G u=1$ and $v$ is a nilpotent element then $u v=v u=0$.

Proof. If $u v \neq 0$ then $u v=g u=u h$ for some $g, h \in G$. Thus $u=g^{-1} u v$ so $u v=g^{-1} u v^{2}$. This is false if $v^{2}=0$. If $v^{2} \neq 0$ then $G v=v G, \operatorname{dim} G v=2$ and $v^{2} \in(G v)^{-} \mid G v$. Also, $u v \in(G v)^{-}$but $u v \notin G v$. For if $u v \in G v$, then $g^{-1} u v^{2} \in G v$ while $v^{2} \in(G v)^{-} \mid G v$ and $(G v)^{-} \mid G v$ is an ideal. But $u v^{2}=(u v) v=0$ by Theorem 6.3 , which implies here th th $\left((G v)^{-} \backslash(G v)\right) . G v=\{0\}$. In any case, $u v=0$. Since the hypotheses are symmetrical, $v u=0$ also.

3. If $x$ and $y$ are nilpotent then $x y x=y x y=0$. In particular, if $x$ and $y$ are nilpotent, $(x y)^{2}=0$. 
Proof. If $x^{2}=y^{2}=0$ we already have this result. Suppose $x^{2}=0$ but $y^{2} \neq 0$. Then $x y \in(x G)^{-}$so $x y x=0$. On the other hand, $x y \in(G y)^{-}$. If $x y \in G y$ then $(x G)^{-} \cap G y$ $\neq \varnothing$ so $x G \cap G y \neq \varnothing$ since $\operatorname{dim} G y=2$. But then $x G \subset G y$, and since $x^{2}=0, y^{2}=0$ which is a contradiction. Therefore $x y \in(G y)^{-} \mid G y$ so $y x y=0$. A similar argument applies if $y^{2}=0$ and $x^{2} \neq 0$. Suppose $x^{2} \neq 0$ and $y^{2} \neq 0$. If $G x=G y$ we know $x y x$ and $y x y$ are zero. If $G x \cap G y=\varnothing$ then $x y \notin G y$ since if so then $(x G)^{-} \cap G y \neq \varnothing$, whence $x G \cap G y \neq \varnothing$. Thus, $x y \in(G y)^{-} \backslash G y$ and similarly $y x \in(G x)^{-} \backslash G x$. The first statement implies $y x y=0$ and the second implies $x y x=0$.

4. If $x$ and $y$ are nilpotent then $G x y=x y G$ and if $x y \neq 0$ then $\operatorname{dim} G x y=1$.

Proof. If $x^{2}=0=y^{2}$, we already have this result. Suppose $x^{3}=0$ but $x^{2} \neq 0$. Then $\operatorname{dim} G x=\operatorname{dim} x G=2$ and $(G x)^{2} \subset A$ where $A$ is a left and right $G$-orbit in $\partial G x$. Now $x y \in(G x)^{-}$and the desired conclusion follows if $x y \in G x=x G$. Suppose $x y=g x$. If $y^{2}=0$ then $x y^{2}=g x y=0$ which is false. Therefore $y^{2} \neq 0$ and $\operatorname{dim} G y$ $=\operatorname{dim} y G=2$ and $x y \in G x$. Since $x y \in(G y)^{-}, G x \cap G y \neq \varnothing$ so $G x=G y$. But then $x y \notin G x$ since $(G x)^{2} \subset A$.

That the product of any three nilpotent elements is zero now follows. For suppose $x, y, z \in N$. Then either $x y=0$, or $G x y=x y G, \operatorname{dim} G x y=1$ and $(x y)^{2}=0$. Therefore $(x y) z=0$ by 2 above.

For the next result, and subsequently, it is useful to have the following lemma which is of some interest in its own right.

LEMMA 6.6. If $e$ is an idempotent then $\mathrm{Se}=(\mathrm{Ge})^{-}$so Se is closed and either a point, a line, a half-ray, a plane or a half-plane.

Proof. For any element $x, S x=G^{-} x \subset(G x)^{-}$so $S e \subset(G e)^{-}$. Since $e^{2}=e, x e=x$ for all $x \in(\mathrm{Ge})^{-}$so $(\mathrm{Ge})^{-} \subset S e$. The final assertions about the shape of Se come from the fact that every orbit of $G$ is "regularly" embedded.

THEOREM 6.7. For an element $x \in L$, the following conditions are pairwise equivalent:

(1) $S x=S e$ for some idempotent $e$;

(2) $G x=G e$ for some idempotent $e$;

(3) $x S=e S$ for some idempotent $e$;

(4) $x G=e G$ for some idempotent $e$;

(5) $x$ is not weakly nilpotent;

(6) Gx contains no weakly nilpotent elements;

(7) $x G$ contains no weakly nilpotent elements.

Proof. Suppose $S x=S e$. Since $S e$ is closed, $S e=(S e)^{-} \subset(G x)^{-}$, so $e \in(G x)^{-}$. If $e \notin G x$, then $G e \subset(G x)^{-} \mid G x$ so $S e=(G e)^{-} \subset(G x)^{-} \mid G x$ which is a contradiction. Hence, when $S x=S e, e \in G x=G e$. Similarly if $x S=e S$.

Suppose $G x=G e$. Since $G e$ is a semigroup, $x^{2} \in G x$ and $x^{2}$ is not weakly nilpotent.

If $x$ is not weakly nilpotent then there exists an idempotent $e \in G x \cap x G$ so $G x=G e$ and $x G=e G$. 
If $G x=G e$ then $e=g x$ for some $g \in G$ so $S e=S g x=S x$. Similarly if $x G=e G$.

If $x^{2} \in G x \cup x G$ then $G x=G e$ and $x G=e G$ for some idempotent $e \in G x \cap x G$. Since $G e$ and $e G$ are semigroups, no elements of $G x \cup x G$ are weakly nilpotent. The proof of the theorem is complete.

Even though, if $x$ is weakly nilpotent, all elements of $G x \cup x G$ are weakly nilpotent, this need not be true of all elements of $S x \cup x S$. Indeed, the square of a weakly nilpotent element need not be weakly nilpotent. For example, on the set of triples $(a, b, c)$ with $a>0, b \geqq 0$ define $(a, b, c)(x, y, z)=(a x, b y, b z+c y)$. Then $(a, b, c)^{2}=\left(a^{2}, b^{2}, 2 b c\right)$. Thus, if $\alpha=(a, 0, c)$ then $\alpha$ is weakly nilpotent but $G \alpha^{2}$ contains the idempotent $(1,0,0)$ so $\alpha^{2}$ is not weakly nilpotent.

THEOREM 6.8. Let $\mathscr{R}$ denote the closure of the set $W$ of weakly nilpotent elements of $S$. Then $\mathscr{R}$ is a (closed) two-sided ideal of $S$.

Proof. By the results above, if $x \in W$ then $G x \cup x G \subset W$ so $S x \cup x S=G^{-} x$ $\cup x G^{-} \subset(G x)^{-} \cup(x G)^{-} \subset \mathscr{R}$. Thus $S W \cup W S \subset \mathscr{R}$ so $S(W)^{-} \cup W^{-} S \subset(S W)^{-}$ $\cup(W S)^{-} \subset \mathscr{R}$ so $\mathscr{R}$ is a closed two-sided ideal of $S$.

Definition 6.9. The set $\mathscr{R}$ of the theorem is the radical of $S$.

THEOREM 6.10. If $S$ has a two-sided zero and $\mathscr{R} \neq \varnothing$ then $N$ has a nonzero element.

Proof. Let $x$ be an element with $x^{2} \notin G x \cup x G$. Thus $x \neq 0$. If $x^{2}=0$ we are through. If $x^{2} \neq 0$ then $\operatorname{dim} G x=\operatorname{dim} x G=2$ and $x^{2} \in \partial G x$. Let $A$ denote the onedimensional right and left $G$-orbit in $\partial G x$ which contains $x^{2}$. If $\left(x^{2}\right)^{2} \neq 0$ then $A$ is a group so by Theorem 2.10, $\partial G x$ also contains a right and left $G$-orbit $B$. Since $G x=x G$ and $x^{2} \in A, a b \in A$ for all $a, b \in G x$ so $(G x)^{-} \subset A^{-}$. Since $B^{-}$is an ideal, $B^{2} \subset B^{-}$so $B^{2} \subset B^{-} \cap A^{-}=\{0\}$ and $S$ contains a nonzero nilpotent element.

THEOREM 6.11. Suppose $S$ does not contain a two-sided zero. Then the radical $\mathscr{R}$ has one of the following structures (exclusive of left-right duals):

(1) $\mathscr{R}$ is empty;

(2) for some element $x \in W, \operatorname{dim} x G=1, \operatorname{dim} G x=2$ and $\mathscr{R}=G x \cup G e$ where $(x G)^{-}=x G \cup\{e\}$ and $\mathscr{R}^{2}=G e$;

(3) for some pair of elements $x, y \in W, \operatorname{dim} x G=\operatorname{dim} y G=1, \operatorname{dim} G x=\operatorname{dim} G y=2$, $G x \cap G y=\varnothing,(x G)^{-}=x G \cup\{e\},(y G)^{-}=y G \cup\{e\}, \mathscr{R}=G x \cup G y \cup G e=L$ and $L^{2}=G e$;

(4) for some element $x \in W, \operatorname{dim} G x=\operatorname{dim} x G=2$, there is an idempotent $e \in \mathscr{R} \backslash W$ such that $\partial G x=G e=e G, \mathscr{R}=W \cup G e=G x \cup G e$ and $\mathscr{R}^{2}=G e$;

(5) for some elements $x_{1}, x_{2} \in L, \operatorname{dim} G x_{i}=\operatorname{dim} x_{2} G=2$ for $i=1,2, G x_{1} \cap G x_{2}$ $=\varnothing$, there is an idempotent $e$ in the common boundary of $G x_{1}$ and $G x_{2}$ such that $G e=e G$ and $\mathscr{R}=G x_{1} \cup G x_{2} \cup G e=L$ while $\mathscr{R}^{2}=G e$. If $\mathscr{R}$ is of one of the types described in (2)-(4) then $G$ is one of the two groups described in Theorem 2.12. Every group on $E_{3}$ except $\mathrm{Sl}(2)$ is the maximal group of a semigroup on a half-space in which $\mathscr{R}$ is empty (though generally in more than one way). 
Proof. Suppose $\mathscr{R}$ is not empty and let $x \in W$. If $\operatorname{dim} G x=1$ then $\operatorname{dim} x G=2$ (else there is a two-sided zero since $G x$ is not closed). Furthermore, $(G x)^{-}=G x \cup\{e\}$ where $G e=e$ and $\operatorname{dim} e G=1$. Thus by Theorem $2.11, L \mid e G$ is the union of two right orbits. If the right orbit $y G$ of $L \backslash e G$ different from $x G$ is a group then $\mathscr{R}$ is one of the type described in (2). If $y G$ is not a group then as is shown in Theorem 2.12, we may assume $y^{2}=e$ and $\mathscr{R}$ is of the type described in (3).

Suppose no element of $W$ has a one-dimensional left or right orbit and again choose $x \in W$. Then $\operatorname{dim} G x=\operatorname{dim} x G=2$ and since $x^{2} \in(G x)^{-}, x^{2} \in \partial(G x)$. If $\operatorname{dim} x^{2} G=0$ then $x^{2}$ is a left zero and $\operatorname{dim} G x^{2}=1$ since $S$ has no two-sided zero. But then, by Theorem 2.12, $L \backslash G x^{2}$ is the union of two two-dimensional left orbits and it can not happen that both are also right orbits. Furthermore if one is, it is also a group. Therefore $\operatorname{dim} x^{2} G=1$. Similarly, $\operatorname{dim} G x^{2}=1$ and since $S$ has no zero, $G x^{2}=x^{2} G$ is closed and is a group. It may be that $\mathscr{R}=(G x)^{-}$in which case $\mathscr{R}$ is as described in (4). If $\mathscr{R} \neq(G x)^{-}$, let $y \in \mathscr{R} \mid(G x)^{-}$. Then $\operatorname{dim} G y=\operatorname{dim} y G=2$ since neither $G y$ nor $y G$ is closed and if either is one-dimensional, there is a left or a right zero which is false. But then $y^{2} \in y G$ so $y$ actually belongs to $W$ and $y^{2} \in(G x)^{-}$. Thus $\mathscr{R}$ is as described in (5).

To show that every group on $E_{3}$, other than $\mathrm{Sl}(2)$, is the maximal group of a semigroup on a half-space, we appeal to the next section, wherein it is shown that every group with a normal one-parameter subgroup is the maximal group of such a group with $L$ a group. The only group on $E_{3}$ which does not contain such a subgroup is the following:

$$
\left(\begin{array}{ccc}
\rho^{t} \cos \theta & \rho^{t} \sin \theta & x \\
\rho^{-t} \sin \theta & \rho^{t} \cos \theta & y \\
0 & 0 & 1
\end{array}\right), \quad 0<\rho<1
$$

and it is obvious that the closure of this group yields a half-space semigroup.

7. Radical free semigroups. In this section, we determine all possible multiplications on the boundary of half-space semigroups which have empty radical. In addition, examples are offered of each possibility. As we have indicated before, it is our belief that the examples offered exhaust the types that include them but this belief is not settled here.

THEOREM 7.1. Suppose $S$ does not have a two-sided zero and that the radical of $S$ is empty. Then L has one of the following forms or has the left-right dual of one of these forms when this makes sense:

(1) $L$ is a group;

(2) $L$ is left trivial (i.e. $x y=x$ for all $x, y \in L$ );

(3) for some idempotent $f, f G=f$ and $\operatorname{dim} G f=1$ while one of the right orbits of $L \mid G f$ is a group and the other has the form: $\operatorname{dim} G e=2, \operatorname{dim} e G=1$ for some idempotent $e$ (as described in Theorem 2.12); 
(4) for some idempotent $f, f G=f, \operatorname{dim} G f=1$ and the two right orbits of $L \backslash G f$ are each generated by idempotents with one-dimensional left orbits;

(5) $L$ is a rectangular band: $\{x y\}=x G \cap G y$;

(6) $L=G e$ and $\operatorname{dim} e G=1$ for some idempotent $e$;

(7) $L$ is the union of three groups $G e_{1}, G e_{2}$ and $G f$ where $e_{1}, e_{2}, f$ are idempotents and $\operatorname{dim} G e_{i}=\operatorname{dim} e_{i} G=2$ for $i=1,2$ while $\operatorname{dim} G f=\operatorname{dim} f G=1$. Thus $G f$ forms the common boundary of $\mathrm{Ge}_{1}$ and $\mathrm{Ge}_{2}$. Moreover, $G$ is either commutative, isomorphic to $R \times \operatorname{Af}(1)$ or $G$ is one of the semidirect products $R \cdot V_{2}$ which has at least two conjugacy classes of noncommutative planar groups (but $G$ may be hyperbolic).

Proof. Since the radical is empty, every left as well as every right orbit of $G$ is generated by an idempotent. Suppose there exists an idempotent $e$ such that $G e=L$. If $e G=L, L$ is a group. If $\operatorname{dim} e G=1$, we have case (6) while case (2) occurs if $\operatorname{dim} e G=0$. If there exists an idempotent $f$ with $\operatorname{dim} G f=1$ and $\operatorname{dim} f G=0$ then (the dual of) one of the situations described in Theorem 2.12 obtains. The only ones of these with empty radical correspond to cases (3) and (4).

Suppose none of the previous cases nor their duals occurs. If $\xi_{12} \cup \xi_{21} \cup \xi_{22}$ is empty then $\operatorname{dim} G e \leqq 1$ and $\operatorname{dim} e G \leqq 1$ for every idempotent $e$. By the cases already excluded, we may suppose $\operatorname{dim} G e=\operatorname{dim} e G=1$ for all $e$. Then $G e \neq e G$ and $L$ is a rectangular band by Theorem 2.5. For if $G e=e G$ for all $e$ then $G e$ is closed for all $e$ or else there is a two-sided zero. But if $G e$ and $f G$ are closed then $G e \cap f G \neq \varnothing$ so since $f G=G f, G e=G f$ which is a contradiction.

Thus, we may suppose there exists an idempotent $e$ with $\operatorname{dim} G e=2$ but $G e \neq L$. By Theorem 2.10, we may suppose $\partial(G e)$ is a group since the other possibilities lead to one of the cases already considered or to the existence of a two-sided zero. Therefore $e G=G e$ since if $\operatorname{dim} e G=1, \partial(G e)$ contains a left zero. Similarly, if $e_{2}$ is an idempotent in $L \backslash(G e)^{-}$then $G e_{2}=e_{2} G$ and $G e_{2}$ and $G e$ have a common boundary and one of the cases in (7) obtains. Since $e$ and $e_{2}$ commute, $e e_{2}$ is an idempotent so $e e_{2}=f$. Let $H=G_{l}(f)$. Since $e f=f=e_{2} f, e_{1}$ and $e_{2}$ belong to $H^{-}$. [To see that $e_{1} \in H^{-}$, write $G=P H$ where $G f=P f$. Let $p_{n} h_{n} \rightarrow e_{1}$. Then $p_{n} h_{n} f \rightarrow e_{1} f$ so $p_{n} f \rightarrow f$. But then $p_{n} \rightarrow 1$ so $1 / p_{n} \rightarrow 1$ so $h_{n} \rightarrow e$ and $e \in H^{-}$.] Thus $H$ is a normal commutative planar group and if $P_{i}=G_{l}\left(e_{i}\right)$ then $P_{i} \subset H$ and $P_{i}$ is normal in $G$. Therefore $G$ has one of the forms given in (7).

We show next that each of the possibilities mentioned in the theorem actually occurs.

Each group $G$ on $E_{3}$ which contains a normal one-parameter subgroup $P$ is the maximal group of a semigroup on a half-space in which $L$ is a group. To see that this is so, we notice the following very general situation, alluded to in [6].

Suppose a one-parameter group $P$ acts as a transformation group on a locally compact space $X$ so that $X$ is a fiber bundle over the space $X / P$ of orbits of $P$. Define a topology on $X \cup X / P$ as in [6]. This space is Hausdorff and when $X$ satisfies the second countability axiom is homeomorphic to $P^{-} \times X / P$ [11]. 
Let $G$ be one of the groups on $E_{3}$ containing a normal one-parameter subgroup $P$. Then $G$ is a fiber bundle over $G / P$ so $G \cup G / P$ is a half-space. Define a multiplication on $G \cup G / P$ as follows: multiplication in $G$ is that already given. If $g$ is an element of $G$ and $P x$ is an element of $G / P$, define $g \cdot P x=P(g x)$ and $(P x) g=P(x g)$. Define $P x P y=P x y$. It is not difficult to prove that this multiplication is continuous in $G \cup G / P$. We thus have

Proposition 7.2. Let $G$ be a group on $E_{3}$ containing a normal one-parameter subgroup $P$. With multiplication as defined above, $G \cup G / P$ becomes a semigroup on $a$ half-space with maximal group $G$ and $L$ isomorphic to $G / P$.

Examples in which multiplication on $L$ is left trivial were offered in Theorem 2.13. It may be of interest that these can be constructed, using the topological properties of $G \cup G / P$, defining multiplication by:

$$
\begin{array}{ll}
P x P y=P x, & x, y \in G, \\
g(P x)=g P, & g, x \in G, \\
(P x) g=P x g, & g, x \in G,
\end{array}
$$

and searching out conditions on $P$ that make this multiplication be continuous.

An example in which $L$ is as in case (3) of the theorem is provided by $P^{-} \times \operatorname{Af}(1)^{-}$:

$$
(a, b, c)(x, y, z)=(a x, b y, b z+c), \quad a, b, x, y \geqq 0 .
$$

In this case, if $f=(0,0,0), f G=f$ and $\operatorname{dim} G f=1$. If $e=(0,1,0), \operatorname{dim} G e=\operatorname{dim} e G=2$. If $e_{2}=(0,1,0)$ then $\operatorname{dim} G e_{2}=2$ and $\operatorname{dim} e_{2} G=1$.

An example in which $L$ is as in case (4) is provided by

$$
(a, b, c)(x, y, z)=(a x, a c y+b, c z), \quad a, c, x, z \geqq 0 .
$$

Here, take $f=(0,1,0), e_{1}=(0,1,1)$ and $e_{2}=(1,1,0)$. While there may be entirely natural ways of arriving at this example, we were led to it by trying to construct it "intrinsically", beginning with $G^{-} \cup G^{-} / P$ where $G^{-}=R \times \operatorname{Af}(1)^{-}$and $P$ is a one-parameter subgroup of $G$ other than the center and not contained in $\{0\} \times \operatorname{Af}(1)$.

We have already listed a class of semigroups in which $L$ is a rectangular band (these were given following Theorem 2.5).

For an example in which $L$ contains an idempotent $e$ with $L=G e$ and $\operatorname{dim} e G=1$, take $P \times \operatorname{Af}(1)^{-}$(or $R \times \operatorname{Af}(1)^{-}$, of course):

$$
(a, b, c)(x, y, z)=(a x, a y+b, c z), \quad a, x>0, b, y \geqq 0 .
$$

An example of the case in (7) with $G$ commutative is provided by the subsemigroup of elements of $P^{-} \times P^{-} \times P^{-}$with $x>0$. An example with $G$ isomorphic to $R \times \operatorname{Af}(1)$ is provided by $P^{-} \times H^{-}$where $H^{-}$is the semigroup on a half-plane with $H=\operatorname{Af}(1)$ and $\partial H$ a group. 
To obtain examples as in (7) with $G=R \cdot V_{2}$, note the following result:

If $\mathscr{T}$ is a semigroup of endomorphisms of a semigroup $T$ then we may form the holomorphic extension of $T$ by $\mathscr{T}$ exactly as in the theory of groups. Namely, define

$$
\left(t_{1}, \tau_{1}\right)\left(t_{2}, \tau_{2}\right)=\left(t_{2}\left(t_{1} \tau_{2}\right), \tau_{1} \tau_{2}\right)
$$

We apply this to the following situation: Choose a one-parameter group of endomorphisms of $P^{-} \times P^{-}$. Such may be constructed as follows: Let $(a, b)$ be fixed ordered pair of not identically zero real numbers. If $\lambda>0$ and $(x, y) \in P^{-} \times P^{-}$ (where $P^{-}$here denotes the multiplicative semigroup of nonnegative real numbers), define $(x, y)^{A_{\lambda}}=\left(x^{\left(\lambda^{a}\right)}, y^{\left(\lambda^{b}\right)}\right)$. The map $\lambda \rightarrow A_{\lambda}$ yields a one-parameter group of endomorphisms of $P^{-} \times P^{-}$. The holomorphic extension of $P^{-} \times P^{-}$by $\left\{A_{\lambda}\right\}_{\lambda}$ leads to the following definition of multiplication:

$$
\left(x_{1}, x_{2}, \lambda_{1}\right)\left(y_{1}, y_{2}, \lambda_{2}\right)=\left(x_{1}^{\left(\lambda_{2}^{a}\right)} y_{1}, x_{2}^{\left(\lambda_{2}^{b}\right)} y_{2}, \lambda_{1} \lambda_{2}\right)
$$

for $x_{1}, x_{2}, y_{1}, y_{2} \geqq 0$ and $\lambda_{1}, \lambda_{2}>0$.

Here, $(1,1,1)$ is an identity and $(0,0,1),(1,0,1)$ and $(0,1,1)$ are the only idempotents. $L$ consists of all $\left(x_{1}, x_{2}, \lambda\right)$ with $x_{1}=0$ or $x_{2}=0$. If $e_{1}=(1,0,1)$ then $\left(x_{1}, x_{2}, \lambda\right)(1,0,1)=\left(x_{1}, 0, \lambda\right)=(1,0,1)\left(x_{1}, x_{2}, \lambda\right)$ so $G e_{1}=e_{1} G$ and $\operatorname{dim} G e_{1}=2$. Similarly if $e_{2}=(0,1,1)$. On the other hand if $f=(0,0,1)$ then $\operatorname{dim} G f=\operatorname{dim} f G=1$.

Examples have now been given of each of the possibilities listed in Theorem 7.1. We turn to the case in which $S$ has a two-sided zero.

THEOREM 7.3. If $S$ has a zero and no nilpotent elements then $S$ is commutative.

Proof. First, $\mathscr{E}_{12} \cup \mathscr{E}_{21}=\varnothing$. For suppose there exists $e \in \mathscr{E}_{21}$. Of course $0 \in(e G)^{-}$ so 0 belongs to the closure of every conjugate of $e G$. By Theorem $2.10, \partial(G e)$ is a line. Let $T=\partial(G e)$. Since there are no nilpotent elements, $T \backslash\{0\}$ is the union of two groups. Let $f$ be the identity of one of these groups. Let $K=G_{l}(f) ; K$ is a normal planar group and $K^{-}$must have points in $G e$. If $k$ is the component of $\left(K^{-} \cap L\right) \mid\{f\}$ in $G e, k$ is a one-parameter group. The only such groups in $G e$ are conjugate to $e G$ and all of these run to 0 which is a contradiction. The argument is similar for $\mathscr{E}_{12}$ so $\mathscr{E}_{12} \cup \mathscr{E}_{21}=\varnothing$.

From this fact, the fact that every orbit is generated by an idempotent and the fact that the number of conjugacy classes of idempotents is finite (Theorem 4.6), it follows that $\bigcup\left\{G e: e \in \mathscr{E}_{22}\right\}$ is dense in $L$. In fact, $\left|\mathscr{E}_{22}\right| \geqq 3$ which implies that $G$, and hence $S$, is commutative.

For suppose $\left|\mathscr{E}_{22}\right| \leqq 2$. Since if $e \in \mathscr{E}_{22}, \partial G e$ is a line by Theorem 2.10 again, $\left|\mathscr{E}_{22}\right|=2$. Let $e_{1}, e_{2} \in \mathscr{E}_{22}$. For $i=1,2,\left(G e_{i}\right)^{-}$is a semigroup on a half-plane with zero and no nilpotent elements so $G e_{i}$ is commutative and there are two nonzero idempotents $f_{1}, f_{2}$ in the common boundary $T$ of $G e_{1}$ and $G e_{2}$. Furthermore, $e_{1}$ and $e_{2}$ belong to the center of $S$ so $e_{1} e_{2}$ is idempotent. Suppose $e_{1} e_{2}=f_{1}$. Let $\left\{x_{n}\right\}_{n}$ be a sequence in $G e_{1}$ converging to $f_{2}$ and let $\left\{y_{n}\right\}_{n}$ be a sequence in $G e_{2}$ converging to 
$f_{2}$. On the one hand $x_{n} y_{n} \in G e_{1} G e_{2}=G f_{1}$, while on the other, $x_{n} y_{n} \rightarrow f_{2} f_{2}=f_{2}$ which is impossible. Thus $e_{1} e_{2} \neq f_{1}$ and a similar argument shows $e_{1} e_{2} \neq f_{2}$. If $e_{1} e_{2}=0$ then the $x_{n}, y_{n}$ above satisfy: $x_{n} y_{n}=0$. Thus $f_{2}^{2}=0$ which is also impossible. It follows that $\left|\mathscr{E}_{22}\right| \geqq 3$ as claimed and the proof of the theorem is complete.

We conclude with a class of examples of commutative, radical free semigroups with zero. Among other things, they show that even though $\left|\mathscr{E}_{22}\right|$ is finite, $\left|\mathscr{E}_{22}\right|$ can be arbitrarily large (and indeed, assume any value other than 0,1 or 2).

Let $n$ be a fixed positive integer greater than 2. We will construct a semigroup in which $\left|\mathscr{E}_{22}\right|=n$ and which is a subsemigroup of $\left(P^{-}\right)^{n+3}$ where, in general, if $T$ is a semigroup and $k$ is an integer, $T^{k}$ denotes the product semigroup on the $k$-tuples of members of $T$.

The construction is given in terms of a certain set of $n$ linear functions $f_{1}, \ldots, f_{n}$ on $R^{3}$. The functions should have the following properties:

(1) any set of three of them are linearly independent;

(2) if $Z_{i}$ denotes the zero set of $f_{i}$ then the component of $R^{3} \backslash \bigcup_{i=1}^{n} Z_{i}$ where every $f_{i}$ is negative is contained in the octant $\Gamma$ of $R^{3}$ where all coordinates are negative. One can think of this component as an infinite polyhedron $\Pi$ whose faces are contained in the various $Z_{i}$ 's. Furthermore, it is intended that no $f_{i}$ be any multiple of any of the projections on $R^{3}$ so the faces of $\Pi$ are, except for the origin, contained also in $\Gamma$. That such functions can always be found is easy to see. For example, let $\pi_{n}$ be an $n$-sided polygon contained in a disc $C$ in $\Gamma$ which is not contained in a plane through the origin. Order the vertices of $\pi_{n}$ cyclically. Join each vertex to the origin by a line. If $L_{i}, L_{i+1}$ denotes a consecutive pair of such lines, let $Z_{i}$ denote the plane spanned by $L_{i}, L_{i+1}$. For each $i$, choose a linear function $f_{i}$ whose zero set is $Z_{i}$ and which is negative on the side of $Z_{i}$ containing, for example, the center of the disc $C$. The functions $f_{1}, \ldots, f_{n}$ have the desired properties and the construction of the example proceeds with a fixed set of such functions in mind.

Let $H$ be the following subset of $R^{n+3}$ :

$$
H=\left\{\left(x, f_{1}(x), \ldots, f_{n}(x)\right): x \in R^{3}\right\}
$$

Let $\exp$ denote the usual exponential map from $R$ to $P$ and let $\operatorname{Exp}\left(x_{1}, \ldots, x_{i+3}\right)$ $=\left(\exp x_{1}, \ldots, \exp x_{n+3}\right)$. Let $K=\exp H$ and let $K^{-}$be the closure of $K$ in $\left(P^{-}\right)^{n+3}$. The semigroup $K^{-}$is the desired semigroup.

Let $L$ denote the boundary of $K^{-}$; i.e., $L=K^{-} \backslash K$. Note that $L$ consists of those points of $\left(P^{-}\right)^{n+3}$ all of whose coordinates are zero except for a "cyclic pair"; a "cyclic pair" being either a pair " $i, i+1$ " or " $3, n+3$ " and $i>3$. In particular, all elements of $L$ have their first three coordinates zero. For if $x \in L^{-} \backslash L$ and $i \leqq 3$, $x_{i}=\lim \exp x_{i n}$ where $\left\{x_{i n}\right\}_{n}$ is a sequence of real numbers. If any one of the sequence of coordinates of $\left(x_{1 n}, x_{2 n}, x_{3 n}\right)$ has a finite limit at least one of the sequences $f_{j}\left(x_{1 n}, x_{2 n}, x_{3 n}\right), j=1, \ldots, n$, has $+\infty$ for limit since the sequence $\left(x_{1 n}, x_{2 n}, x_{3 n}\right)$ must eventually lie outside the polyhedron $\Pi$. 
Thus $L$ is the union of a "cyclic" collection of "sectors", each of which is topologically a half-plane so that $L$ is obviously a plane.

The points lying in the boundary of the sets $\operatorname{Exp} L_{i}$ are idempotents in $\mathscr{E}_{22}$ so $\left|\mathscr{E}_{22}\right|=n$. (If $\left\{x_{n}\right\}_{n}$ is a sequence in $L_{i}$ becoming negatively infinite, $\lim _{n} f_{j}\left(x_{n}\right)=-\infty$ if $j \neq i, i+1$, when $i<n$, and $\lim _{n} f_{j}\left(x_{n}\right)=0$ if $j=i, i+1$. Thus $\lim _{n} \operatorname{Exp}\left(x_{n}\right)$ has 1 in the $i$ th and $(i+1)$ th place and zero elsewhere.)

To see that $K^{-}$is homeomorphic to a half-space, let $T$ be the line $\{(t, t, t): t \in R\}$ and let $P=\operatorname{Exp} T$. Let $D=(\operatorname{Exp} \Pi)^{-}$. It is straightforward to prove that $D$ is a disc. Let $\Sigma$ be the half-space $\{(x, y, z): x \geqq 0\}$. Write each $x \in \Sigma$ in the form $p \cdot u$ where $p$ is a positive real number and $u$ is a point of the unit hemisphere $E$ in $\Sigma$. Identify $E$ with $D$ by a homeomorphism $h$. The map $(p, d) \rightarrow p d$ is a homeomorphism from $P \times D$ onto $K^{-} \mid\{0\}$, so the map $p \cdot u \rightarrow p \cdot h(u)$ is a homeomorphism from $\Sigma \mid\{(0,0,0)\}$ onto $K^{-} \mid\{0\}$ which extends naturally to a homeomorphism from $\Sigma$ onto $K^{-}$.

Appendix. This section is devoted to a proof of the result which, in the main body of the work, frequently allowed us to conclude that the closure of a planar group is a half-plane. In view of the examples in [3], this result is not entirely expected. According to these examples, it is possible to put a given semigroup on a line and a given planar group together to form nonisomorphic semigroups on a half-plane. Nevertheless, according to the principal result of this section, there is only one topological possibility for a semigroup which is the union of a plane and a line.

THEOREM. Let $S$ be a locally compact Hausdorff topological semigroup whose maximal group is a planar group $G$ which is dense in $S$. If $S \backslash G$ is a line then $S$ is topologically a half-plane, while if $S \backslash G$ is topologically a half-ray then $S$ is topologically a plane.

We treat the cases in which $S$ has or does not have a two-sided zero 0 separately. Suppose $S$ does not have a two-sided zero. Let $x \in L$. Since any endpoint of $G x$ in $L$ is a right zero and any endpoint of $x G$ is a left zero and since a semigroup can not have a left zero and a right zero without having a two-sided zero, either $G x=x G=L$ and $L$ is a group or $G x=x$ and $x G=L$ or $x G=x$ and $G x=L$.

In [3], it was proved that if a semigroup on a half-plane has no nilpotent elements then $G$ and $L$ determine $G \cup L$. However, once it is known that a one-parameter subgroup $P$ and an idempotent $e \in L$ can be found such that $P^{-}=P \cup\{e\}$ and that $P^{-}$is homeomorphic to $[0, \infty)$, the arguments given there are valid knowing only that $G$ is planar, $L$ is a line and $G \cup L$ is locally compact. Moreover, when there is no zero, the result actually proved is the following: there exist one-parameter groups $P, Q$ such that $G \cup L=P^{-} Q$ and the map $(p, q) \rightarrow p q$ is a homeomorphism from $P^{-} \times Q$ onto $G \cup L$. Therefore, if we can prove under our present hypotheses that there exists an idempotent $e \in L$ and a one-parameter subgroup $P \subset G$ such that $P^{-}=P \cup\{e\}$ and that $P^{-}$is homeomorphic to $[0, \infty)$, we may conclude that the theorem is true when $S$ does not have a two-sided zero. 
When $S$ has no zero, we may assume $L$ contains an idempotent $e$ such that $G e=L$. Let $P$ be the component of the identity of $G_{l}(e)$. Then $e P=e$ since either $e G=e$ or $G e=e G=L, L$ is a group and $e$ belongs to the center of $S$. Let $Q$ be a one-parameter subgroup having no conjugates in $P$. Then $G=P Q=Q P$. Let $\left\{x_{n}\right\}_{n}$ be a net in $G$ converging to $e$. Write $x_{n}=q_{n} p_{n}$ with $q_{n} \in Q, p_{n} \in P$. Then $q_{n} p_{n} \rightarrow e$, so $q_{n} p_{n} e \rightarrow e$, whence $q_{n} e \rightarrow e$. Since the map $q \rightarrow q e$ is a homeomorphism from $Q$ to $Q e, q_{n} \rightarrow 1$. It follows that $p_{n} \rightarrow e$ so $e \in P^{-}$. But $P e=e$ so $e$ is a zero for $P^{-}$, whence $P^{-}=P \cup\{e\}$.

It remains to prove that $P^{-}$is homeomorphic to $[0, \infty)$. (In effect, this result is the one-dimensional version of the theorem.) To do this, we prove a lemma which turns out to be needed later in another connection.

Lemma. Let $T$ be a locally compact, connected Hausdorff topology on $[0,1)$ whose restriction to $(0,1)$ is the ordinary topology. Then $T$ is either the ordinary topology on $[0,1)$ or $([0,1), T)$ is homeomorphic to a simple closed curve.

Proof. Since $[0,1)$ is $T$-connected and $(0,1)$ is open, $0 \in(0,1)^{-}$. Therefore $0 \in(0,1 / 2)^{-}$or $0 \in(1 / 2,1)^{-}$. There is no loss in generality in assuming the former. Since closed intervals in $(0,1)$ are $T$-closed, it follows that $0 \in(0, a)^{-}$for all $a \in(0,1)$. We assert that every $T$-neighborhood of 0 contains a set of the form $[0, a)$ for some $a$. If this is false, then there is a $T$-neighborhood $V$ of 0 such that $V^{-}$is compact and no component open interval of $V \cap(0,1)$ abuts 0 . Furthermore, since $V \cap(0, a) \neq \varnothing$ for all $a \in(0,1)$, it is possible to choose a sequence $x_{n}$ of endpoints of component intervals of $V \cap(0,1)$ such that $x_{n} \rightarrow 0$ in the ordinary topology of $[0,1)$. Since $V^{-}$is compact, we may suppose $x_{n}$ converges in the $T$-topology to some point $x \in V^{-}$. But $x$ is obviously not in $(0,1)$ so $x_{n} \rightarrow 0$ in the $T$-topology. Now there is a $T$-neighborhood $W$ of 0 such that $W^{-\subset} \subset$. If $U$ is a component open interval of $W \cap(0,1)$ there is a component open interval $Z$ of $V \cap(0,1)$ such that $U \subset Z$. Now $U$ cannot abut at 0 so $0 \notin U^{-}$. Since $U^{-} \subset V$, $U^{-} \subset Z$. Therefore $x_{n} \notin U$ for any $n$. However, since $W$ is a neighborhood of 0 and $x_{n} \rightarrow 0, W \cap(0,1)$ must contain all $x_{n}$ for $n$ sufficiently large, which is a contradiction. We have shown that $T$-neighborhoods of 0 contain ordinary neighborhoods of 0 .

Now, if $0 \notin\left(\frac{1}{2}, 1\right)^{-}$then obviously $T$ coincides with the ordinary topology. If $0 \in\left(\frac{1}{2}, 1\right)^{-}$then reasoning of the previous type shows that every neighborhood of 0 contains a set of the form $(a, 1)$ for some $a \in(0,1)$. In this case, $([0,1), T)$ is homeomorphic to a simple closed curve.

Let $A, B$ denote the two components of $P \backslash\{1\}$ and assume $0 \in A^{-}$. By the lemma, $A \cup\{0\} \cup\{1\}$ is an arc. If $0 \in B^{-}$then $B \cup\{0\} \cup\{1\}$ is an arc and $P^{-}$is a simple closed curve which is false since $P$ can be embedded in no compact semigroup. It follows that $P^{-}$is homeomorphic to $[0, \infty)$ and, as we have already indicated, conclude the theorem is proved in case $S$ does not have a zero. 
Throughout the remainder of this section, then, assume that $S$ has a two-sided zero 0 . Let $x$ be a nonzero member of $L$. Then $G x=x G$ so either $x G$ is a group or $(x G)^{2}=0$. Therefore 0 is not a limit point of idempotents. Hence there exists a one-parameter subgroup $P$ of $G$ such that $P^{-}=P \cup\{0\}$. Let $X=S \backslash\{0\}$. Let $P$ act on $X$ by left multiplication. By [6], $X$ is a fiber bundle over the space $X / P$ of left cosets of $P$ and $X / P$ is a Hausdorff space.

Since $G / P$ is homeomorphic to a line, $G / P \cup\{P x\}, x$ a nonzero member of $L$, satisfies the conditions of the lemma. Thus, since $X / P$ is Hausdorff, if $L$ is a line, $X / P$ is an arc. If $L$ is a half-ray, $X / P$ is either homeomorphic to $[0,1]$ or is a simple closed curve. In either case, $X$ must satisfy the second countability axiom so there is a complete cross-section $A$ to the orbits of $P$ in $X$ [11]. Let $J$ denote the arc of $P^{-}$from 0 to 1 . Let $D=J \cdot A$. Then $D$ is a neighborhood of 0 . For suppose there is a net $\left\{z_{n}\right\}_{n}, z_{n} \in G$ converging to 0 . There exists $p_{n} \in P$ such that $p_{n} z_{n} \in A$. If $z_{n} \notin D$ then $p_{n} \in J$ so we may suppose $p_{n} \rightarrow p \in P^{-}$. But then $p_{n} z_{n} \rightarrow 0$ which is a contradiction since $0 \notin A$.

Evidently $\bigcap\{t D: t \in J, t \neq 0\}=\{0\}$. For assume $x$ is a nonzero element of this intersection. Then for every $t$, there exists $t_{x} \in J, a_{x} \in A$ such that $x=t \cdot t_{x} \cdot a_{x}$. However, $x=t_{0} a_{0}$ for unique $t_{0} \in P, a_{0} \in A$ so $t \cdot t_{x}=t_{0}$ and $a_{x}=a_{0}$ for every $x$. Since we may choose a sequence $t_{n} \rightarrow 0$ and the corresponding $t_{x}$ 's are bounded, this is impossible.

Let $V$ be a compact neighborhood of 0 . Then there exists $t \in J, t \neq 0$, such that $t D \subset V$. Now $t A \subset t D$ and $t A$ is closed so $t A$ and hence $A$ is compact. It follows that in case $L$ is a half-ray then $X / P$ is a simple closed curve.

We complete the proof of the theorem in case $L$ is a line. The argument in case $L$ is a half-line is similar and is omitted. Choose a homeomorphism $h_{1}$ from $A$ onto the unit semicircle in the right half cartesian plane. Let $h_{2}$ be a homeomorphism from $P^{-}$onto the nonnegative $x$-axis. If $x \in X, x=p a$ for unique $p \in P, a \in A$. Let $h(x)=h_{2}(p) h_{1}(a)$ (complex multiplication). Let $h(0)$ be the origin. Then $h$ is a one-to-one and continuous function which is a homeomorphism on $X$. Furthermore, $h$ maps the compact neighborhood $D$ above onto the unit half-disk so $h$ is a homeomorphism on $D$ and hence a homeomorphism everywhere.

\section{REFERENCES}

1. J. G. Horne, Jr., Real commutative semigroups on the plane, Pacific J. Math. 11 (1961), 981-997. MR 25 \#2586a.

2. - One-parameter subgroups in semigroups in the plane, Michigan Math. J. 9 (1962), 177-186. MR 25 \#2587.

3. - Semigroups on a half-plane, Trans. Amer. Math. Soc. 105 (1962), 9-20. MR 25 \#5502.

4. - The boundary of a one-parameter group in a semigroup, Duke Math. J. 31 (1964), 109-117. MR 28 \#3404.

5. - A locally compact connected group acting on the plane has a closed orbit, Illinois $\mathbf{J}$. Math. 9 (1965), 644-650. MR 31 \#5921. 
6. J. G. Horne, Jr., Flows that fiber and some semigroup questions, Notices Amer. Math. Soc. 13 (1966), p. 820.

7. N. Jacobson, Lie algebras, Interscience Tracts in Pure and Appl. Math., vol. 10, Interscience, New York, 1962. MR 26 \#1345.

8. P. S. Mostert and A. L. Shields, Semigroups with identity on a manifold, Trans. Amer. Math. Soc. 91 (1959), 380-389. MR 21 \#4204.

9. G. D. Mostow, The extensibility of local Lie groups of transformations and groups on surfaces, Ann. of Math. (2) 52 (1950), 606-636. MR 14, 18.

10. B. J. Pettis, Uniform Cauchy points and points of equicontinuity, Amer. J. Math. 73 (1951), 602-614. MR 13, 217.

11. N. E. Steenrod, The topology of fiber bundles, Princeton Math. Series, vol. 14, Princeton Univ. Press, Princeton, N. J., 1951. MR 12, 522.

12. H. Whitney, Regular families of curves, Ann. of Math. (2) 34 (1933), 244-270.

UNIVERSITY OF GEORGIA,

Athens, Georgia 